
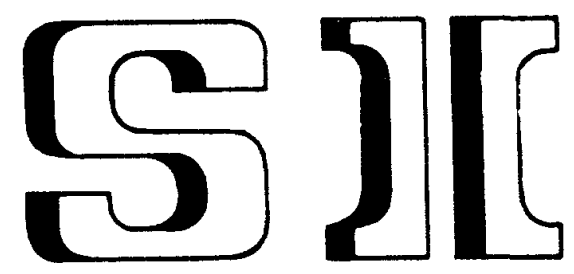

GSI-94-16

PREPRINT

MÄRZ 1994

ELECTROMAGNETIC EXCITATION OF THE TWO-PHONON GIANT DIPOLE RESONANCE

H. EMLING

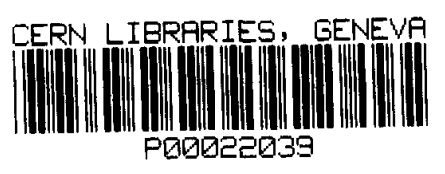

(Accepted for publication in 'Progr. Part. Nucl. Phys.', Vol. 3 (1994))

Gesellschaft für Schwerionenforschung $\mathrm{mbH}$ Postfach 110552 · D-64220 Darmstadt · Germany 



\title{
ELECTROMAGNETIC EXCITATION OF THE TWO-PHONON GIANT DIPOLE RESONANCE
}

\author{
Hans Emling \\ Gesellschaft für Schwerionenforschung $\mathrm{mbH}$, \\ Planckstr. 1, 64291 Darmstadt, Germany
}

\section{CONTENTS}

1. Introduction

2. Brief History and Preliminaries

2.1 Giant Resonances - a Short Reminder

2.2 Giant Resonance Studies using Heavy Ion Beams

2.3 Phenomenology of the Giant Dipole Resonance

2.4 Multi-Phonon States - Preliminary Remarks

3. Electromagnetic Excitation in Relativistic Heavy Ion Collisions
3.1 Semi-Classical Methods
3.2 Multi-Step Excitations
3.3 Nuclear Fragmentation

4. Experimental Studies of the Double Giant Dipole Resonance
4.1 Activation Measurements
4.2 Exclusive Measurements and Neutron Decay
$4.3 \gamma$-Decay Studies
4.4 Pion Double Charge Exchange Reactions
4.5 Two-Phonon Isoscalar Giant Quadrupole Resonance

5. Discussion
5.1 Summary of the Experimental Data
5.2 Strength Distribution
5.3 Neutron and $\gamma$ Decay
5.4 Cross Sections

6. Summary and Outlook

References 



\section{INTRODUCTION}

Amazingly, after almost fifty years of nuclear giant resonance studies, a new class of giant resonances has been discovered only rather recently, i.e. their multi-phonon states. Out of those, the two-phonon isovector giant dipole resonance and the two-phonon isoscalar giant quadrupole resonance are now experimentally established. Even more amazing is the fact that these new giant resonances are observed with exceptionally large cross sections resulting from a purely electromagnetic process, the Coulomb interaction between heavy ions swiftly passing each other. Such an excitation mechanism provides a 'clean' probe which allows for an accurate determination of strength distributions.

Giant resonances appear as resonant structures in the multipole transition strength distribution of nuclei and exhaust a large fraction of it. They are observed in almost any nucleus an.: their characteristic parameters vary only smoothly with nuclear mass. They thus represent a very elementary mode of a nuclear collective motion and might be viewed as small-amplitude density oscillations around equilibrium density in the nuclear interior. If these oscillations are basically harmonic, higher-phonon states of nearly equidistant energy spacing should exist. In a microscopic picture, giant resonances are understood as a coherent superposition of one-particle one-hole excitations, forming the phonon, in response to an external force. Multi-phonon giant resonances are then interpreted as collective $n p-n h$ states. For harmonic oscillations the vidrational amplitude increases approximately as $\sim \sqrt{n}$ ( $n \equiv$ number of phonons). The basic question related to multi-phonon giant resonance states is that of the nuclear response to an induced collective motion of increasing amplitude. It is the question of how and if the restoring forces can balance, and to which extent the frictional forces do allow for such vibrations of increasing amplitude. Anharmonicities might arise resulting from interactions between the phonons through modifications of the mean field and residual interactions. Finally, it has to be explored to which extent standard theoretical methods, developed for small amplitude motions, are still appropriate in the case of multi-phonon excitations. First signs of a two-phonon isovector giant dipole resonances were obtained from pion double charge exchange reactions (Mordechai et al., 1988; 1990). Total cross sections are small, amounting to a few $\mu \mathrm{b}$, and the double giant dipole resonances are superimposed on a large, not well understood background.

In recent experiments, however, the heavy ion induced electromagnetic excitation of the double dipole resonance was experimentally observed at cross sections up to about $500 \mathrm{mb}$ (LAND, 1991; Schmidt et al., 1993; Ritman et al., 1993a). These cross sections exceed those obtained from pion double charge exchange by about a factor $10^{5}$, and thus give access to very detailed studies, for example of weak decay branches. Moreover, an extremely clean signal is obtained, since the excitation mechanism is rather selective and background, if any, is well under control. The results were achieved using heavy ions of near relativistic energies at the SIS facility of GSI, Darmstadt.

With the advent of accelerators being capable of delivering ion beams even of the heaviest elements such as $\mathrm{Pb}$ or $\mathrm{U}$, the technique of Coulomb excitation has rapidly been developed into a standard method in nuclear spectroscopy. A large part of the presently 
available information on low-lying collective nuclear states originates in studies utilising heavy ion induced Coulomb excitation at subbarrier bombarding energies. The beauty of the method is two-fold, at one hand the relevant cross sections are large, on the other hand the excitation process can theoretically be described to almost perfect correctness since nuclear interaction is not involved. The excitation probabilities are ruled by the strength of the Coulomb field, i.e. the nuclear charge of the interacting heavy ion, as well as by the magnitude of the electromagnetic multipole moments of the excited nucleus. Using the heaviest beams, excitation probabilities for low-lying collective states might even approach unity, giving rise to multi-step excitations, a mechanism widely exploited in the past in studies of high spin states. For example, excitations within the groundstate rotational band of deformed actinide nuclei, involving up to 15 steps of multipolarity $\lambda=2$ and leading to a spin as high as $\mathrm{I}^{\pi}=30^{+}$, were observed (Ower et al., 1982).

Such data are collected employing beam energies, in most cases, slightly below the Coulomb barrier ( $\sim 5 \mathrm{~A} \cdot \mathrm{MeV})$ in order to avoid nuclear contact between the colliding heavy ions. The relative velocitv between the ions, consequently, is relatively small, typically around $v \approx 0.1 \mathrm{c}$, and the adiabaticity of the process imposes a limit on the available excitation energy to below $1-2 \mathrm{MeV}$. A large fraction of the multipole transition strength in nuclei, however, is concentrated at much higher energies $(\sim 10-30 \mathrm{MeV})$, i.e. in the domain of giant resonances. This region of excitation energies is thus not accessible in low energy Coulomb scattering.

Raising the beam energies into the A.GeV regime, i.e. $v \gtrsim 0.8 c$, removes the adiabaticity limit and high-lying collective states will be excited. Since giant resonances are unstable against decay via particle emission, this process is often referred to as 'Coulomb dissociation' or 'Coulomb fragmentation'. In fact, it was observed that in energetic heavy ion collisions Coulomb dissociation contributes considerably to the total reaction cross section. The excitation probability for giant resonances thus are large, and estimates show that multi-step excitations are likely to occur, in close analogy to the multiple excitations within rotational bands obtained at low beam energies. At relativistic bombarding energies, multi-step processes give access to multi-phonon states of giant resonances.

It is the aim of this article to summarize our present knowledge on the double isovector giant dipole resonance (DGDR) and our understanding of the electromagnetic excitation mechanism in heavy ion collisions in the relativistic energy regime. In the following chapter, a brief résumé on the history of giant resonances is given and, based on their understanding, conclusions on the expected properties of multi-phonon resonances are drawn. In chapter 2, the essential features of electromagnetic heavy ion interactions at (near) relativistic velocities will be illuminated and the theoretical framework is presented, which describes such processes. New experimental methods were required for an appropriate study of Coulomb dissociation processes, which are discussed in chapter 3 together with the experimental results. Chapter 4 is dedicated to summarize the results from electromagnetic excitation studies, to compare with those from alternative methods and, in particular, to contrast experimental findings with theoretical predictions and to address open problems.

It must be clearly stated, that our present knowledge is based on experimental information obtained from very recent measurements, so to i.e. from a first round of exper- 
iments. It is obvious that more detailed studies are still required before reaching a complete understanding.

Short reviews on the subject of double giant dipole resonances were presented earlier (Mordechai and Moore, 1991; Fortune, 1991; Emling, 1993a, 1993b).

\section{BRIEF HISTORY AND PRELIMINARIES}

\subsection{Giant Resonances - a Short Reminder}

Extended literature exists on experimental and theoretical investigations of the phenomena of nuclear giant resonances. It would go far beyond the scope of the present article to attempt an adequate review of the subject. One of the most comprehensive and up-to-date summaries is presented in (Speth, 1991), a collection of five review articles to which the reader seeking for a complete survey is referred. Earlier articles on selected aspects are found in (Berman and Fultz, 1975; Bertrand, 1976; Pitthan, 1979; Snover, 1986; van der Woude, 1987).

The nuclear excitation spectra exhibit a rich variety of collective modes. The classification schemes which are usually applied, first distinguish surface and volume modes. The former ones comprise surface vibrations and rotations, while giant resonances belong to the latter class, as they are understood to manifest density oscillations in the interior of a nucleus. Secondly, depending on whether the two constituents of the nucleus, i.e. protons and neutrons, vibrate in phase or out of phase one deals with isoscalar and isovector excitations, respectively. Since nucleons carry spin, coherent spin excitations may occur as well, and thus giant resonances of electric and magnetic nature exist. Finally, the transition multipolarity of the collective mode serves to classify giant resonances.

Historically, the first observation related to giant resonances was made already in 1937 when Bothe and Gentner exposed targets to energetic photons of $17.6 \mathrm{MeV}$ from the ${ }^{7} \mathrm{Li}$ $(p, \gamma)$ reaction and unexpectedly found a large photoproduction of radioactivity in some of the targets, an effect which led them to believe in a resonant photoabsorption process. A firm proof of a resonant photoabsorption at high excitation energies was achieved about ten years later by Baldwin and Klaiber (1947), exploiting the development of the betatron by which photons of sufficiently high energy could be produced. This was the discovery of the isovector giant dipole resonance (GDR), one of the most elementary nuclear modes, where neutrons vibrate against protons. Progress in searching for more complex giant resonances and in studying more details of their structure was closely linked to advanced techniques, in particular to the development of new types of accelerators and powerful magnetic spectrometers. Only in the early 1970's a new class of giant resonances, the isoscalar quadrupole resonances, were found in inelastic electron scattering (Pitthan and Walcher, 1971) and proton scattering (Lewis and Bertrand, 1972), somewhat later the giant monopole resonances was discovered. From this 'breathing' mode a fundamental property of nuclear matter, its compression modulus, was derived. 
Successively, electric transitions of higher multipolarites were investigated, complicated by the increasing fragmentation of their transition strength. Another chapter of giant resonances is that of magnetic resonances with the various spin-isospin degrees of freedom, a subject which cannot be discussed here.

The various giant modes are widely overlapping each other in excitation energy and in order to disentangle them, selective probes are required. The most selective tool is photoabsorption which almost exclusively leads to isovector excitations of multipolarity $\lambda=1$. Electron scattering is the other tool extensively used in giant resonance studies. It is the virtue of the electromagnetic interaction between electron and nucleus that it is rather insensitive to transition multipolarity and does not distinguish between isoscalar and isovector strength, but it is its drawback at the same time, since rather complicated spectra arise. Since the form factors exhibit patterns characteristic of the transition multipolarity, the contributions from different multipolarities, however, can be separated in principle.

Hadronic probes such as protons or He used in inelastic scattering or charge exchange reactions are more selective. The $\alpha$-particle with zero spin and isospin almost exclusively excites isoscalar modes, to the extent that its electromagnetic interaction can be neglected. Since the isoscalar part of the nucleon-nucleon interaction is predominant in a wide range of energies, proton scattering is also useful for isoscalar excitations. In a certain range of proton energies $(\sim 200-400 \mathrm{MeV})$, however, the isovector spin-flip interaction is comparable in magnitude and $(p, n)$ reactions, for instance, were applied in studying magnetic resonances such as the Gamow-Teller resonance. A major problem in using such probes is an adequate description of the underlying background, resulting from quasifree proton scattering, or knock-out and pickup-breakup reactions in the case of the $\alpha$-particle. It was discussed for a long time, how reliably the transition strength can be extracted from measured cross sections in such reactions.

Charge exchange reactions such as $(p, n)$ or $\left({ }^{3} \mathrm{He}, t\right)$ induce isovector transitions. At bombarding energies useful for giant resonances studies, i.e. $>100 \mathrm{~A} \cdot \mathrm{MeV}$, however, the isospin-spin flip part $(\Delta T=1, \Delta S=1)$ of the nucleon-nucleon interaction is dominant over the $\Delta T=1, \Delta S=0$ part and pure isovector excitations are difficult to study. In contrast, the pion with $S=0, T=1$ is well suited for such excitations, since also its spin-orbit interaction is small at forward scattering angles. Secondary pion beams from meson factories as LAMPF are of sufficient intensity allowing such measurements. A major problem consists in the large and not very well controlled continuum underlying the resonances. Nevertheless, from pion double charge exchange reactions, the first observation of the double isovector giant dipole resonance was reported (see chapter 4.4).

The large body of experimental data collected for giant resonances was and still is a challenge for the theoretical description and interpretation. Soon after the discovery of the GDR, a theoretical interpretation of the observed phenomenon was provided by Goldhaber and Teller (1948) on the basis of the hydrodynamical model. They consider the oscillation of rigid proton and neutron spheres against each other, the restoring force resulting from the surface symmetry energy. They also proposed a model, later developed in (Steinwedel and Jensen, 1949), where proton and neutron fluids are 
compressible and vibrate with opposite phase in one common fixed sphere, here the restoring force results from the volume symmetry energy. One of the basic assumptions in hydrodynamical models is that of a short mean free path of nucleons inside a nucleus, which thus should encounter frequent collisions. Due to Pauli blocking, however, the mean free path of the nucleon is of the order of the nuclear dimension and thus models of independent particle motion in a mean field might be a more appropriate approach. The Random Phase Approximation (RPA) which is the standard theoretical method for the description of giant resonances, in fact can be derived from transport equations based on the time-dependent Hartree-Fock method in the limit of small amplitudes. Thus, in a natural way the shell structure of the finite nucleus can be implemented and the structure of giant resonances is understood on a microscopic basis. In the microscopic picture, giant resonances appear as coherent superpositions of one-particle one-hole (1p1h) excitations in closed shell nuclei or two quasi-particle excitations in open shell (deformed) nuclei. RPA calculations were rather successful in describing mean energies and transition strength of giant resonances. Difficulties, however, arise in describing the accurate strength distribution. In particular, since by construction based on collisionless transport equations, the RPA cannot account for the collisional damping resulting in a spreading width. Extended RPA calculations take into account $2 \mathrm{p} 2 \mathrm{~h}$ excitations, the coupling of which to the $1 \mathrm{p} 1 \mathrm{p}$ excitations is considered to be the most important term as they act as doorway states to more complex configurations $(3 p 3 h, .$.$) . Since such ap-$ proaches yield a large configuration space, certain truncation schemes and simplifications are invoked, e.g. residual interactions are diagonalized in the $1 \mathrm{p} 1 \mathrm{~h}$ and $2 \mathrm{p} 2 \mathrm{~h}$ subspaces separately. Nevertheless, such schemes were applied in studies of twophonon excitations. Another concept is that of sumrules, decomposing the strength distribution into their moments, the lowest ones describing total strength, mean energy and so.on. For some of those moments, rather model independent values can be derived. A comprehensive discussion of the various theoretical techniques, their success and open problems is presented in (Speth, 1991).

\subsection{Giant Resonances Studies using Heavy Ion Beams}

Giant resonance studies with heavy ion beams is a relatively young field. Early attempts were made employing inelastic scattering of lighter ions such as carbon (Kamermans et al., 1979), oxygen or neon (Buenerd et al., 1986; Bertrand et al., 1987) at moderate energies of $\sim 10-30 \mathrm{~A} \cdot \mathrm{MeV}$. At those energies, nuclear interaction is prevailing and, as in the case of $a$-particles, a large continuum is present. When raising the bombarding energy to about $100 \mathrm{~A} \cdot \mathrm{MeV}$, Coulomb excitation dominates and yields large cross sections at small scattering angles. The spectra are composed to most extent out of the excitation to the isovector giant dipole resonance (GDR) and the isoscalar giant quadrupole resonance (ISGQR), both observed with cross sections of the order of several b/sr. Although the Coulomb excitation mechanism does not distinguish between isoscalar and isovector 
Fig. 1a. Spectra obtained at small laboratory scattering angles from inelastic scattering ${ }^{208} \mathrm{~Pb}\left({ }^{17} \mathrm{O},{ }^{17} \mathrm{O}\right)$ at $84 \mathrm{~A} \cdot \mathrm{MeV}$ bombarding energy (Barrette et al., 1988). A decomposition into resonance and continuum structures is indicated (solid lines).

Fig. 1b. Angular distributions for the GDR $(L=1)$ and the ISGQR $(L=2)$ observed in the same reaction. Calculations for the differential cross section (solid line) and its decomposition into nuclear and Coulomb part are also shown.

Fig. 1c. Inelastic spectra for scattering of ${ }^{17} \mathrm{O}$ on ${ }^{208} \mathrm{~Pb}$ at 22 and $84 \mathrm{~A} \cdot \mathrm{MeV}$ and for ${ }^{36} \mathrm{Ar}$ on ${ }^{201} \mathrm{~Pb}$ at $95 \mathrm{~A} \cdot \mathrm{MeV}$. The spectra are normalized to be equal at an excitation energy of $35 \mathrm{MeV}$ (Beene, 1993).
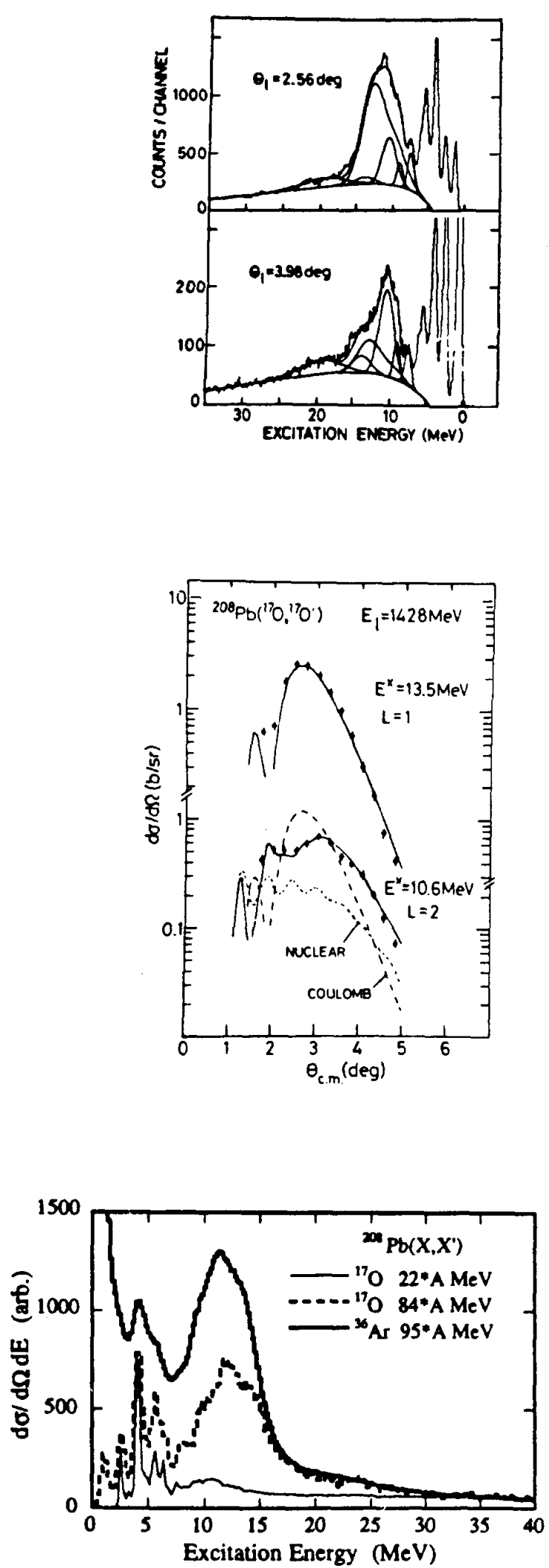
modes, the isovector giant quadrupole resonance (IVGQR) is suppressed because of its higher excitation energy. Fig. 1a and $1 \mathrm{~b}$ show examples of inelastic scattering spectra obtained with "O ions at small angles and corresponding angular distributions (Barrette et al., 1988). The angular distributions are not very sensitive to transition multipolarity, at least for its Coulomb part, as seen from Fig. 1b. The virtue of energetic heavy ion beams comes basically from the sizeable, Coulomb induced cross section, which improves drastically the ratio of resonance signal to continuum. This effect is demonstrated in Fig. 1c (Beene, 1993). The large cross sections in turn make more-than-inclusive experiments feasible, and for example even weak decay branches can be studied (Beene et al., 1990).

A major progress achieved by means of heavy ions was the observation of the GDR in nuclei at high temperature and angular momentum. The first relevant observation was made by (Newton et al.,1981) measuring the high-energy part of $\gamma$ de-excitation spectra after a heavy ion fusion reaction. Until then, only giant resonances built on the nuclear groind state had been studied, except for a few cases, where the GDR built on discrete low-lying states was observed in radiative proton-capture experiments (Dowell et al., 1983). The complete fusion of two heavy ions leads to a compound nucleus in a region of high nuclear level density, but in this process the excitation energy and, with additional experimental provisions, also the transferred angular momentum is well under control. The decay of the highly excited nucleus is basically of statistical nature, and in heavy nuclei proceeds predominantly via neutron emission and eventually by fission. Nevertheless, the GDR high-energy $\gamma$ decay can compete although on a $10^{-4}-10^{-5}$ branching only. Observing this branch, the GDR properties in nuclei at high temperature and spin were deduced. Up to temperatures of about $2 \mathrm{MeV}$ only minor effects are observed with regard to strength and resonance energy; an increase in width is attributed to shape changes or fluctuations as an combined effect of increased spin and temperature. For an overview of this field we refer to (Snover, 1986; Gaardhoje, 1992).

\subsection{Phenomenology of the GDR}

In this chapter experimentally well established facts on properties of the isovector giant dipole resonance are summarized. We choose to do so in order to have all prerequisites available, eventually required for an understanding of the two-phonon GDR, the subject of this article.

Now studied for almost five decades, the GDR certainly is the best-known giant resonance. Unlike other resonances, an highly selective excitation mechanism is available, i.e. photoabsorption. Photoabsorption experiments nowadays utilize tagged monochromatic $\gamma$ rays from electron beam bremsstrahlung or from in-flight electron-positron annihilation. For heavier nuclei, to which the discussion is restricted, data are usually obtained from coincidence measurements with neutrons or fission fragments; charged particle decay of the GDR is strongly suppressed by the Coulomb barrier. Below the neutron threshold, data are more difficult to obtain since the resonant fluorescence is overwhelmed by elastic $\gamma$-ray scattering. As an example, Fig. 2 contains the 
photoabsorption spectrum for ${ }^{208} \mathrm{~Pb}$. A compilation of GDR data from photoabsorption measurements and the deduced empirical systematics is provided in (Berman and Fultz, 1975; Berman, 1975). In spherical nuclei the E1 strength distribution with regard to excitation energy $E$ can be parametrized using a lorentzian form

$$
\sigma_{\text {phot. }}(E)=\frac{2 \sigma_{o}}{\pi \Gamma\left(1+\left(E^{2}-E_{o}^{2}\right)^{2} / E^{2} \Gamma^{2}\right)},
$$

where $\sigma_{0}, E_{0}$, and $\Gamma$ denote the integrated cross section, resonance energy (more precisely: energy of maximum cross section) and full width at half maximum, respectively. In order to fit photo-neutron data, a gaussian cut-off has to be applied to the lorentzian near the neutron threshold. The lowest moment of the distribution, i.e. the total photo absorption cross section, is usually compared to the Thomas-Reiche-Kuhn (TRK) sum rule (Levinger, 1960)

$$
j \sigma_{\text {phot. }}(E) d E=\frac{60 \mathrm{NZ}}{A} \quad[\mathrm{MeV} \cdot \mathrm{mb}]
$$

In heavy nuclei, the cross section integrated up to $25 \mathrm{MeV}$ excitation energy exceeds the TRK sum rule by typically 10-20\%. Apparent E1 strength at higher energies, interpreted in terms of a quasi-deuteron absorption mechanism, appears structureless and remains nearly constant up to the pion threshold. The energy $E_{0}$ of maximum cross section follows a mass dependence (Berman and Fultz, 1975)

$$
E_{\mathrm{o}}=31.2 A^{-1 / 3}+20.6 A^{-1 / 6} \quad[\mathrm{MeV}] .
$$

Such a dependence indicates that a mixture of the Steinwedel-Jensen (SJ) and the Goldhaber-Teller (GT) modes (see subsection 2.1) contributes, the former one gaining weigth with increasing mass. A combination of $\mathrm{GT}$ and $\mathrm{SJ}$ modes was derived in a more rigorous treatment of the GDR in the hydrodynamical droplet model (Myers et al., 1977).

A splitting of the GDR strength can occur through isospin effects and due to coupling to the nuclear deformation. Photoabsorption on a nucleus $(N \neq Z)$ with isospin $\left(T, T_{z}\right)=\left(T_{0}, T_{0}\right)$ can populate states $T_{<}=\left(T_{0}, T_{0}\right)$ and $T_{\rangle}=\left(T_{0}+1, T_{0}\right)$ which are separated by several $\mathrm{MeV}$ in excitation energy. Estimates for the energy difference $E_{>}-E_{<}$ (Akyüz and Fallieros, 1971) and for the relative strengths $S_{>} / S_{<}$(Fallieros and Goulard, 1970) are

$$
\begin{gathered}
E_{>}-E_{<}=60(T+1) / \mathrm{A}[\mathrm{MeV}] \\
\frac{S_{>}}{S_{>}}=\frac{1}{\mathrm{~T}} \frac{1-(3 / 2) \mathrm{TA} \mathrm{A}^{-2 / 3}}{1+(3 / 2) \mathrm{A}^{-2 / 3}}
\end{gathered}
$$

Correspondingly, in heavier neutron rich nuclei the $T_{<}$component is predominantly excited, e.g. for ${ }^{208} \mathrm{~Pb}$ one obtains $S_{>} / S_{<}=2.6 \cdot 10^{-3}$ and $E_{>}-E_{<}=6.6 \mathrm{MeV}$. In axially deformed nuclei, a splitting of the GDR is observed, since vibrations parallel or perpendicular to the nuclear symmetry axis are possible. The coupling of the GDR to surface modes was considered in the dynamical collective model (Eisenberg and Greiner, 1970). 


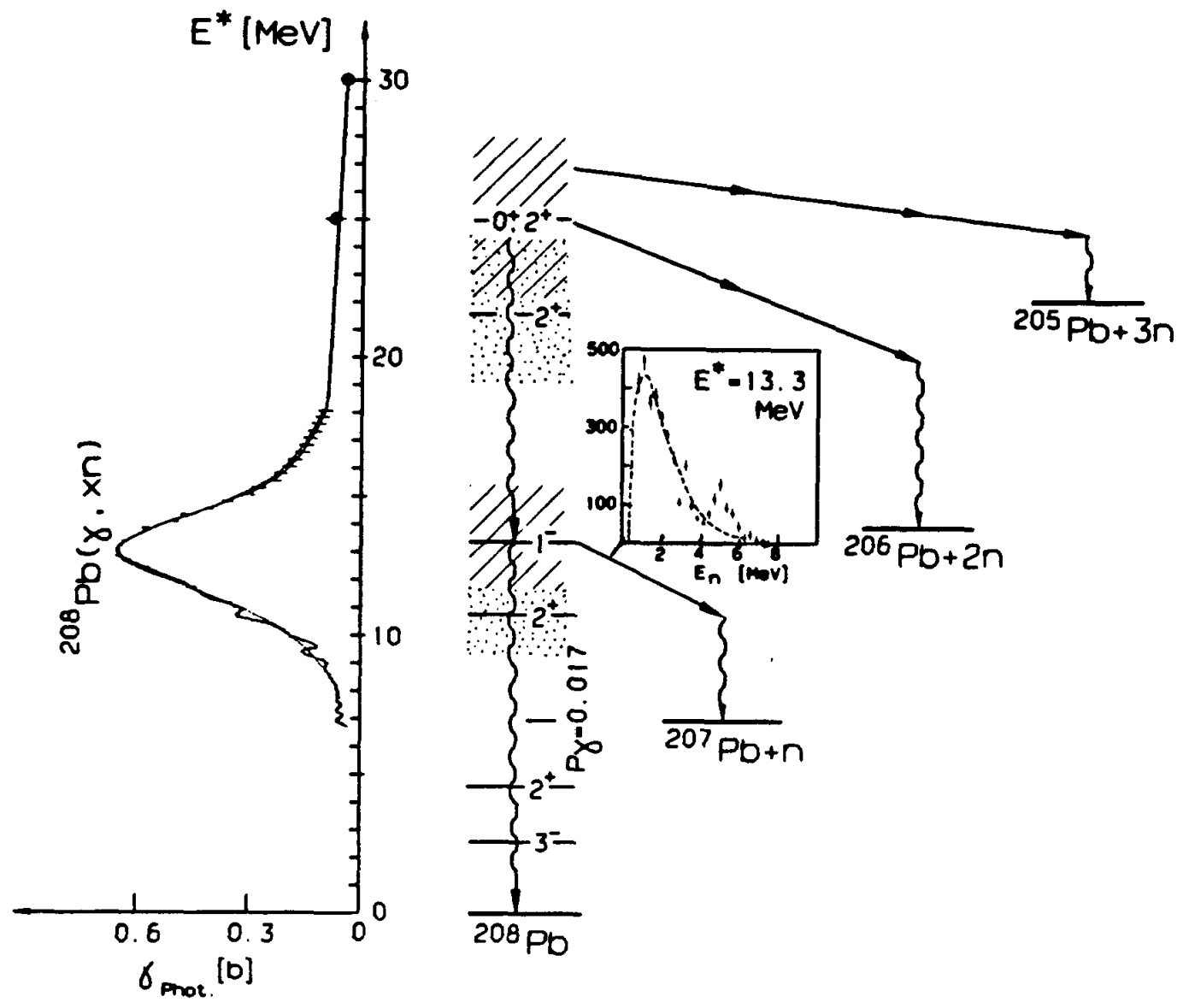

Fig. 2. Empirical information available for the GDR $\left(1^{-}\right)$in ${ }^{208} \mathrm{~Pb}$. The photoabsorption cross section $\sigma_{\text {phot }}$ is from (Veyssière et al., 1970; Schelhaas et al., 1988), the neutron-decay energy spectrum at an excitation energy of $13.3 \mathrm{MeV}$ from (Cardman, 1981), the $y$-decay branch from (Beene et al., 1990). Giant quadrupole resonances $\left(2^{+}\right)$taken from data systematics (Bertrand, 1976; van der Woude, 1991) and a few low-lying discrete states are also indicated. In addition, the two-phonon GDR $\left(0^{+}, 2^{+}\right)$and possible decay paths are tentatively shown.

For an axially deformed nucleus with a difference $\Delta R$ between major and minor axis, a GDR resonance energy splitting was deduced to $\Delta E=0.45 \Delta R / R$ (Jang, 1983).

The width of the GDR is rather independent of nuclear mass and in heavier nuclei, except for cases of resonance splittings discussed above, amounts to values around $\Gamma \approx$ 4-5 MeV where $\Gamma$ corresponds to the width parameter in a lorentzian par umetrization for the photoabsorption cross section. The width is romposed out of three contributions: (i) the fragmentation width resulting from the residual particle-hole interaction, (ii) the escape width $\Gamma^{\dagger}$, since the GDR is particle unstable and thus semi-direct decays into hole states of the $(A-1)$ nucleus may occur, and (iii) the spreading width $\Gamma^{t}$ arising from the mixing of the $1 \mathrm{p} 1 \mathrm{~h}$ states with $2 \mathrm{p} 2 \mathrm{~h}$ and more complicated states, finally reaching equilibrated compound states. $I t$ is generally believed that $\Gamma^{\dagger}$ is small compared to $\Gamma^{t}$. 
In this limit the particle decay of the GDR would exhibit statistical features only. A number of neutron decay studies were performed searching for semi-direct decay components. In heavy nuclei, however, such branches were found to be small and, if at all, amount to $\leqslant 20 \%$ (Cardman, 1981; Alarcon et al., 1991). The electromagnetic $\gamma$-decay branch is also almost negligible, in ${ }^{208} \mathrm{~Pb}$ for instance a $1.7 \%$ branch of direct $\gamma$ decay back to the ground state was observed (Beene et al.,1990). In Fig. 2 the empirical information available for the GDR (to some extent also for higher multipolarities) is presented for the specific case of ${ }^{208} \mathrm{~Pb}$. This double magic nucleus is probably the one studied at best, both on experimental and theoretical side.

\subsection{Multi-Phonon States - Preliminary Remarks}

Giant resonances are small amplitude motions, the GDR droplet model (Myers et al.,1977) for example yields amplitudes amounting to a few percent of the nuclear dimensions. To lowest order one thus might rely on the harmonic approximation. Therefore, the basic features of harmonic vibrations are shortly recalled.

The quantum mechanical treatment of a harmonic oscillator can be found in any textbook on quantum dynamics, for example (Schiff, 1968; Bohr and Mottelson, 1975). The spectrum of equidistant excitation energies $E_{n}$ is the characteristic feature of the linear harmonic oscillator, i.e.

$$
E_{n}=(n+1 / 2) h \omega_{o},
$$

where $n$ denotes the number of phonons and $\omega_{0}$ the eigen frequency. The dynamics of the system can be described by a wave packet whose center of gravity oscillates with the frequency $\omega_{0}$ which is equal to that of the corresponding classical harmonic vibration. In the limit of large $n$, moreover, the amplitude $A_{n}$ of the oscillating wave packet becomes also identical to that of the classical case, i.e.

$$
A_{n} \approx\left(\frac{2}{K} E_{n}\right)^{1 / 2} \quad ; \quad \frac{A_{n}}{A_{1}} \approx\left(\frac{2 n+1}{3}\right)^{1 / 2} .
$$

$K$ denotes the coefficient describing the restoring force. For the oscillator in three dimensions, the multipolarity of the vibrational mode and the spin coupling between the phonons has to be considered taking into account the Bose statistics. For example two phonons carrying spin $L$ can be coupled to total spins

$$
I=0,2, \ldots, 2 L \quad[h] .
$$

In the harmonic approximation, transitions between multi-phonon states obey the selection rule $\Delta n= \pm 1$. Since the phonons are considered to be independent, the electromagnetic decay rate is obtained by summing the decay rate of the individual phonons. With $I_{n}$ denoting the spin and $\xi_{n}$ denoting an additional quantum number needed to distinguish states of same $n$ and $I_{n}$ but resulting from different spin couplings, it was derived (Bohr and Mottelson, 1975) that 


\section{Hermonic Oscillator}

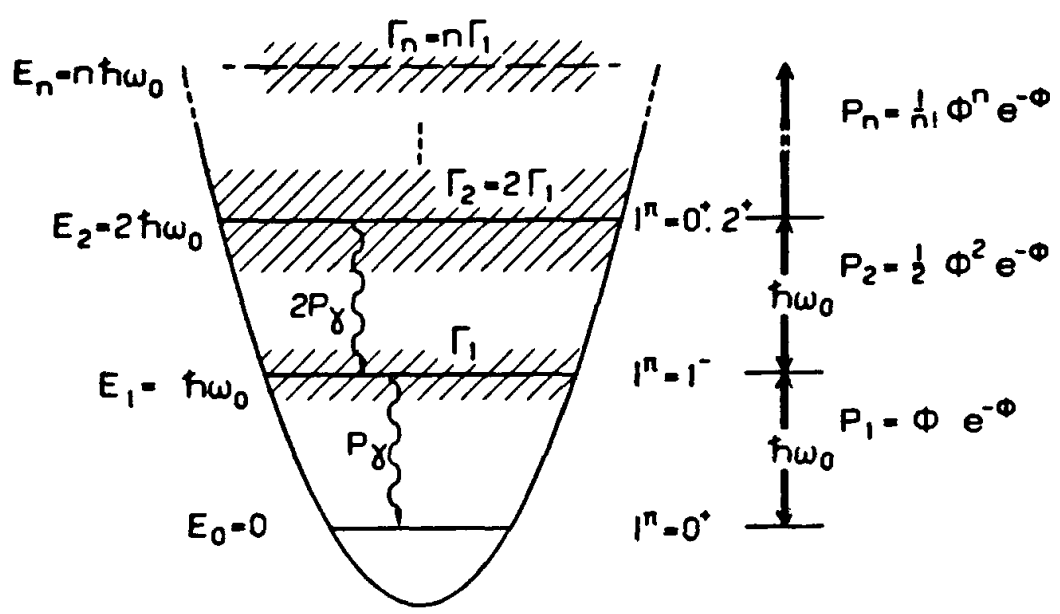

Fig. 3. Multi-phonon states of a linear harmonic oscillator. $P$, and $P_{n}$ denote $\gamma$-decay probabilities and excitation probabilities (e.g. for electromagnetic excitation), respectively. $E_{n}$ and $\Gamma_{n}$ denote excitation energy and damping width, respectively. Spin couplings for $L=1$ (GDR) phonons are also given.

$$
\sum_{\xi_{n-1}, I_{n-1}} B\left(E \lambda ; n, \xi_{n}, I_{n} \rightarrow n-1, \xi_{11-1}, I_{n-1}\right)=n B\left(E \lambda_{;} n=1 \rightarrow n=0\right)
$$

As dicussed above various damping mechanisms lead to a width $\Gamma$ for the giant resonance. If in an n-step excitation mechanism an n-phonor, state is excited and the assumption of independent phonons is valid, the observed width for the $n$-phonon state would be obtained by an n-time folding of the giant resonance shape. If the shape can be described by a lorentzian distribution, which is a good approximation for the GDR in heavy nuclei, this leads to

$$
\Gamma_{n}=n \Gamma_{1}
$$

The validity of above assumptions will be discussed later. Fig. 3 summarizes our expectations for multi-phonon vibrational states resulting from the simple approach of independent quanta of the harmonic oscillator.

The nuclear droplet model (Myers and Swiatecki, 1974) applied to giant dipole resonances ( Myers et. a!., 1977) can be extenoed to the higher-phonon states as outlined in (Gomes and Bertulani, 1990) and also applied in (Emling, 1985). Althougin a macroscopic picture, it may illustrate some of the basic features of multi-phonon GDR states. Fig. 4 displays proton and neutron densities for an $n=4$ phonon state in ${ }^{238} U$ and for an $n=6$ phonon state in ${ }^{40} \mathrm{Ca}$ at the turning point of the dipole vibration. 

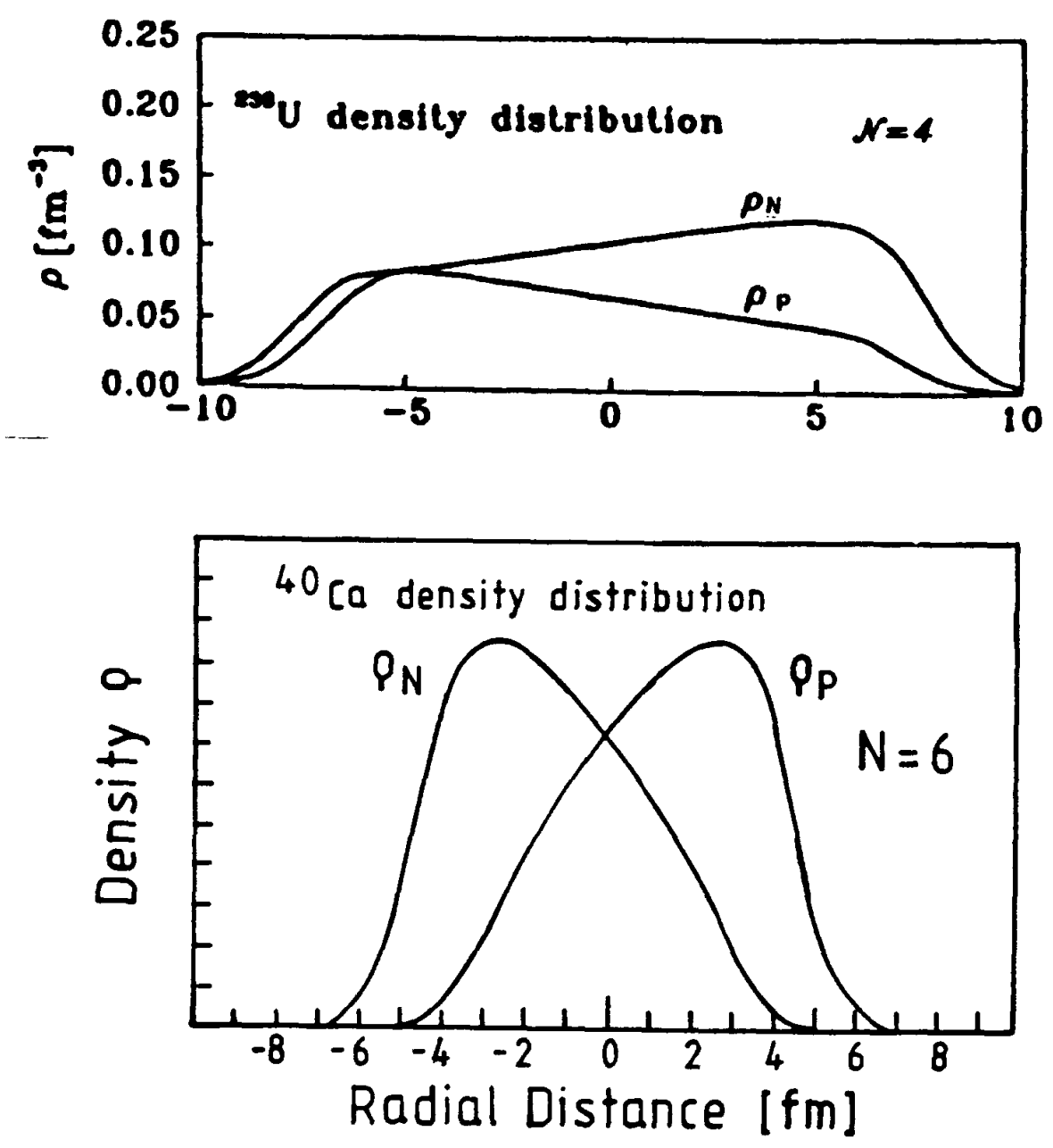

Fig. 4. Proton and neutron densities $\left(\rho_{\rho, n}\right)$ at the instant of their largest separation calculated in the nuclear droplet model. Upper panel is for a $n=4$ phonon GDR state in ${ }^{238} \cup$ (Gomes and Bertulani,1990), lower panel for $n=6$ state in ${ }^{40} \mathrm{Ca}$ (Emling, 1985).

Because of the large instantaneous neutron excess, it was speculated if exotic decays, e.g. neutron cluster emissions, may occur (Gomes and Bertulani, 1990; Emling, 1990). In the extreme case, a 'Coulomb explosion' of nuclei may take place, i.e. two clusters of proton and neutron matter are formed and the nuclear restoring forces cannot counterbalance their separation under the influence of the electromagnetic boost. Conditions under which such a situation may occur were studied in a relativistic $m$ əan field Walecka model (Vretenar et al., 1993).

A final remark of this introduction will be devoted to low-lying surface-vibrational states and to non-linear effects in the nuclear response. 
Table 1. Partial results of the calculation of (Ponomarev and Voronov, 1992a; 1992b) for twophonon giant resonances of configurations as indicated. GDR and ISGQR denote giant dipole and isoscalar giant quadrupole phonons, respectively. $E_{\text {harm }}$ denotes the unperturbed harmonic centroid energy of the two-phonon state, $\Delta E$ denotes the difference between $E_{\text {harm }}$ and the centroid energy resulting from the calculated strength distribution.

\begin{tabular}{|c|c|c|c|}
\hline Nucleus & Configuration & $E_{\text {harm }}[\mathrm{MeV}]$ & $\Delta \mathrm{E}[\mathrm{MeV}]$ \\
\hline${ }^{208} \mathrm{~Pb}$ & $\begin{array}{l}{[\mathrm{GDR} \times \mathrm{GDR}] 2^{+}} \\
{[\mathrm{GDR} \times 1 \mathrm{SGQR}] 1^{-}}\end{array}$ & $\begin{array}{l}26.7 \\
24.0\end{array}$ & $\begin{array}{r}-1.2 \\
1.1 \\
\end{array}$ \\
\hline${ }^{136} \mathrm{Xe}$ & $\begin{array}{l}{[\text { GDR } \times \text { GDR }] 2^{+}} \\
{\left[\text {GDR } \times \text { ISGQR } 1^{-}\right.} \\
{[\text {ISGQR }}\end{array}$ & $\begin{array}{l}30.6 \\
25.6 \\
20.6\end{array}$ & $\begin{array}{r}-0.4 \\
1.6 \\
0.7\end{array}$ \\
\hline
\end{tabular}

Although studied over many years, no clear and no systematic pattern for higher-phonon surface vibrations could be observed. A quantitative consideration of energies and eletromagnetic transition rates for the corresponding spin-multiplets shows that large deviations from harmonicity arise through deformation coupling and admixtures of single particle excitations. Many attempts were made, for example, to localize the twophonon octupole state in ${ }^{208} \mathrm{~Pb}$. Only rather recently, such a double octupole phonon state with spin assignment $l^{\pi}=4^{+}$or $6^{+}$could be identified (Wollersheim et al., 1992) in a Coulomb excitation measurement. The deviation from the harmonic value in excitation energy amounts to about $20 \%$ and it is also noticed, that the other members of the $1^{\pi}=0^{+}, 2^{+}, 4^{+}, 6^{+}$spin multiplet are not observed. In a similar study also a two-phonon state of the quadrupole $\gamma$-vibration in the deformed nucleus ${ }^{232}$ Th was identified (Korten et al., 1993). In the case of the two-phonon octupole state in ${ }^{208} \mathrm{~Pb}$, the coupling between octupole and quadrupole motions was considered. Quantitative calculations (Blomquist, 1970) inferred anharmonicities and an energy splitting of the spin multiplet of the order of $1 \mathrm{MeV}$, qualitatively in accord with the experimental result.

In general, for giant resonances a much closer resemblance to harmonicity is expected (Bohr and Mottelson, 1975). Extended RPA calculations aiming on an estimate of anharmonicity effects for two-phonon giant resonance states were performed for lighter ions such as ${ }^{40} \mathrm{Ca}$ (Catara et al., 1989). In that case, energy shifts and energy splittings of the spin multiplets were found to be below $500 \mathrm{keV}$. Similar siudies were performed for the heavy isotopes ${ }^{136} \mathrm{Xe}$ and ${ }^{208} \mathrm{~Pb}$ (Ponomarev and Voronov, 1992a; 1992b). Twophonon couplings to states of spin $1^{\pi}=1^{-}, 2^{+}$were calculated, taking into account phonons of multipolarity $\lambda=1$ (isovector) and $\lambda=2$ (isoscalar). Their results are presented in Table 1. For the coupling of two GDR $(\lambda=1)$ phonons, which is particular interest here, energy shifts of -0.4 and $-1.2 \mathrm{MeV}$ relative to the unperturbed harmonic value are calculated for ${ }^{136} \mathrm{Xe}$ and ${ }^{208} \mathrm{~Pb}$, respectively. This result shows that anharmonicity effects in two-phonon giant resonance states are expected to be of the order of a few per cent only. 


\section{ELECTROMAGNETIC EXCITATION IN RELATIVISTIC HEAVY ION COLLISIONS}

The great success of low-energy Coulomb excitation studies certainly resulted from the installation of accelerators for even the heaviest elements, but it was of equal importance that a well developed analysis technique based on semi-classical methods was available (Alder and Winther, 1966; 1975). The semiclassical method allows to calculate differential cross sections to high accuracy, even in the case of complex excitation patterns, involving many intermediate steps and various interfering pathways. This can be accomplished, for instance by using the famous Winther-de Boer computer code (Winther and de Boer, 1966). In order to deduce nuclear structure information in terms of electromagnetic matrix elements by comparing calculated and experimental cross sections, supplemental procedures have been developed independently by groups at GSI (Grein et al., 1983) and at Rochester (Czosnyka et al., 1983), which can handle matrix elements as numerous as hundred or more.

The semiclassical method is applicable equally well to electromagnetic excitations in the (near) relativistic regime of bombarding energies, and it will be shown that the treatment in some sense becomes even more simplified at high velocities. In this chapter, semiclassical methods for relativistic energies are discussed and some predictions of cross sections for giant resonances and their multi-phonon states are presented.. Nuclear processes are also discussed, to the extent that they might interfere with electromagnetic excitations. For a more comprehensive review of the various dynamical theories, applicable to electromagnetic processes at large velocities, and of related problems, we refer to (Bertulani and Baur, 1988; and references therein).

\subsection{Semi-Classical Methods}

The basic principle of the semi-classical method is to assume that the ion moves along a classical trajectory, while the excitation process is treated quantum mechanically by integrating the time-dependent Hamiltonian along the trajectory. The assumption of a classical trajectory is well justified, if the De Broglie wavelength

$$
\hbar=\frac{\hbar}{m_{0} \beta \gamma}
$$

is small in comparison to the dimension of the scattered objects. For typical cases of interest here, i.e. a reduced mass $m_{0}=10-100 \mathrm{GeV} / \mathrm{c}^{2}$ and a Lorentz factor $\gamma=\left(1-\beta^{2}\right)^{-1 / 2} \approx 2$, corresponding to an bombarding energy of about $1 \mathrm{~A} \cdot \mathrm{GeV}$, one obtains $\lambda \approx 10^{-2}-10^{-3} \mathrm{fm}$. Quantal diffraction effects, therefore, should be negligible. $A$ major simplification in high--energy Coulomb scattering arises from the fact that the momentum transfer is extremely small in comparison to the total momentum and consequently, projectile scattering angles observed in the laboratory frame are rather close to zero. This permits the use of so-called straight-line approximation. Because of the low momentum transfer, the laboratory coordinate frame and the target-rest frame nearly coincide. For same reasons, the acceleration (radiation) fields in the Lienhard-Wiechert potentials can be neglected, and the electromagnetic fields mutually seen by the two 
ions are obtained by a Lorentz transformation between two inertial systems moving with relative velocity $\vec{\beta}$.

The solution for the time-dependent transversal and longitudinal electromagnetic fields $E_{\mathrm{t}, 1}(t)$ and $B_{\mathrm{t}, 1}(t)$ can be found for example in the textbook on 'Classical Electrodynamics' (Jackson, 1975):

$$
\begin{aligned}
& E_{l}(t)=-Z_{1} e \gamma \beta t /\left(b^{2}+\gamma^{2} \beta^{2} t^{2}\right)^{3 / 2} \\
& \vec{E}_{l}(t)=Z_{1} e \gamma \vec{b} /\left(b^{2}+\gamma^{2} \beta^{2} t^{2}\right)^{3 / 2} \\
& B_{l}(t)=0 ; \vec{B}_{l}(t)=\vec{\beta} \times \vec{E}_{t}
\end{aligned}
$$

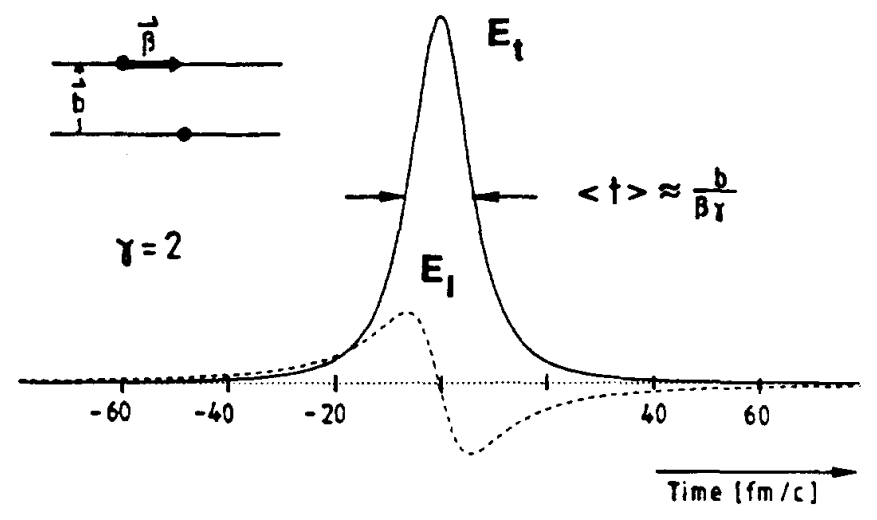

Fig. 5. Time $d \varepsilon$ sendence of the longitudinal and transversal electric field components, induced by a heavy ion passing a target at a distance of $b=15 \mathrm{fm}$ and with a velocity $\beta=0.86$ corresponding to $\gamma=2$. The mean interaction time $\langle t\rangle$ is indicated.

The notation' should become clear from Fig. 5 where the electric fields are evaluated for the particular case of $\gamma=2$ and an internuclear distance $b=15 \mathrm{fm}$. The retardation effects enter via the Lorentz factor $\gamma$, essentially yielding a 'Lorentz contraction' of the electromagnetic field in the direction of the relative velocity $\vec{\beta}$. These retardation effects are usually ignored in calculations at low energy since $\gamma \rightarrow 1$.

Before carrying on, the validity of the straight-line approximation is inspected. For that purpose the Coulomb deflection function has to be evaluated, which provides the link between the classical quantity 'impact parameter' $b$ to the scattering angle $\vartheta$ as an observable. The inelasticity of the processes under consideration is small, since excitation energies are of the order of $10 \mathrm{MeV}$, while the total kinetic energies are of the order of $100 \mathrm{GeV}$. It is thus sufficient to evaluate the elastic deflection function $\vartheta(\mathrm{b})$.

A full relativistic treatment using the exact electromagnetic potential was performed numerically (Matzdorf et al., 1987; 1990); an analytical expression for $\vartheta($ b) cannot be derived. Approximately, however, the transverse momentum transfer $p_{t}$, can be obtained by integrating the action of the transverse electric field over time (Jackson, 1975), i.e.

$$
p_{t}(\mathrm{~b})=\int_{-\infty}^{\infty} z_{2} \in E_{t}(\mathrm{t}) \mathrm{dt}=\frac{2 z_{1} z_{2} e^{2}}{\mathrm{~b} \beta} .
$$
1 Velocity $\vec{\beta}$, time t, momentum $\vec{\rho}$, and mass are measured in units of $c, t / c$, MeV/c, and MeV/c$c^{2}$, respectively, if not stated other-
wise. 
Fig. 6. Deflection function $\vartheta(b)$ evaluated with the approximation given in the text (solid lines) and with the full treatment (symbois) of (Matzdorf et al., 1987; 1990) for the $\mathrm{Pb}+\mathrm{Pb}$ system at different bombarding energies.

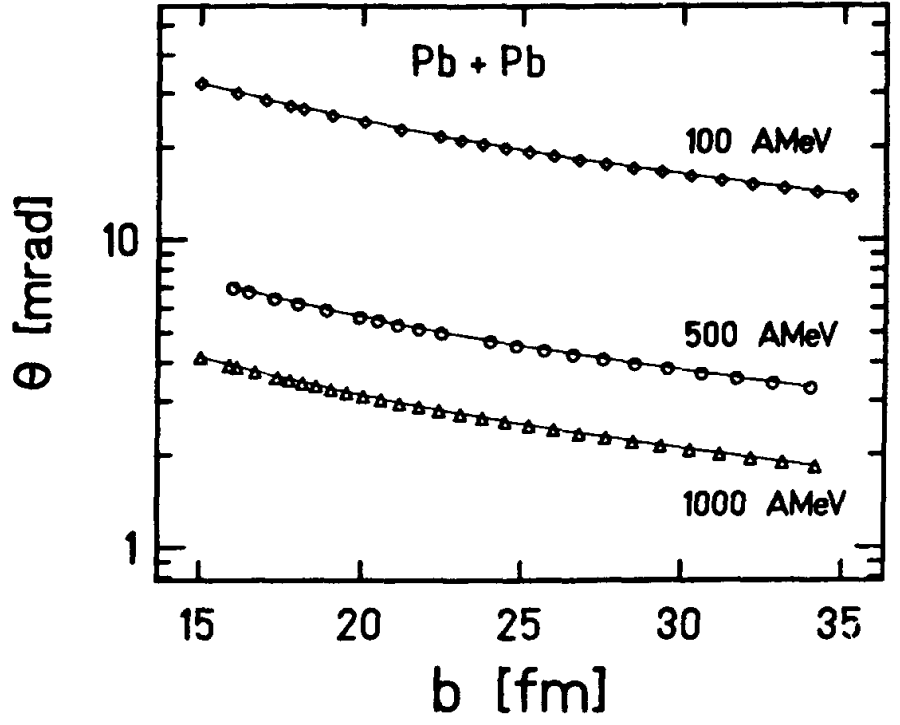

The inverse proportionality to $\beta$ should be noted. The deflection function is formed by comparing $p_{t}$ to the parallel momentum, which is equal to the projectile momentum and thus in the laboratory frame

$$
\vartheta_{\text {Proj. }}(b)=\frac{2 z_{1} z_{2} e^{2}}{b \beta^{2} \gamma m} .
$$

In the limit of small angles, this expression is identical to the classical formula, except that the 'relativistic mass' of the projectile $\gamma m$ replaces $m$. With this modification, also the Rutherford cross section formula should be valid for elastic scattering. In Fig. 6, this approximate deflection function is compared to the full relativistic treatment (Matzdorf et al., 1987; 1990) for a few systems. The differences are obviously negligible for practical cases. Even for grazing collisions with $b=R_{1}+R_{2}$ (sum of the two nuclear radii), scattering angles are of the order of a few milliradian and the straight-line approximation seems to be well justified.

The semiclassical method in the straight-line approximation has first been employed (Winther and Alder, 1979) in a first-order perturbation treatment. Utilizing multipole expansions, closed expressions were derived for differential and total cross sections. Most generally, the excitation probability $\mathrm{P}(\mathrm{b})$ for a transition of multipolarity $\lambda$ between an initial state of spin $l_{1}$ and a final state of spin $l_{\text {, }}$ summed over magrietic substates, can be written as

$$
P(b)=\left(z_{1} \alpha\right)^{2} \quad B\left(\pi \lambda ; \quad l_{i} \rightarrow I_{f}\right) / e^{2} \quad F(\lambda, \beta, \xi)
$$

with

$$
\xi=\frac{\left(E_{\mathrm{f}}-E_{i}\right)}{\hbar} \frac{\boldsymbol{b}}{\beta \gamma} .
$$


The product of nuclear charge $Z_{1}$ and the fine structure constant $\alpha$ governs the strength of the electromagnetic field. $B\left(\pi \lambda, I_{1} \rightarrow I_{f}\right)$ represents the electromagnetic multipole transition moment (for electric transitions $\pi=E$, for magnetic transitions $\pi=M$ ), and the dimensionless function $F$ depends on multipolarity and the dynamical quantitites $\beta$ and the 'adiabaticity parameter' $\xi$, which also involves the nuclear transition energy $E_{f}-E_{i}$ as given above. The function $\mathrm{F}$ drops rather suddenly to zero near $\xi=1$, reflecting the well-known adiabatic cut-off in low-energy Coulomb excitation experiments. The maximum energy which can be transferred in a single step excitation is obtained replacing the impact parameter $b$ by $b_{\min } \approx R_{1}+R_{2}$, below which nuclear absorption becomes predominant. Thus one obtains a cut-off energy

$$
E^{\max }=\left(E_{f}-E_{i}\right)^{\max } \approx \frac{\hbar \beta \gamma}{R_{1}+R_{2}} .
$$

This cut-off energy is simply understood from the reaction dynamics. A Fourier transformation of the electromagnetic fields (see above) yields maximum collisional pulse frequencies which are determined by the mean interaction time $\langle t\rangle \approx\left(R_{1}+R_{2}\right) / \beta \gamma$ which readily leads to the above relation (see also Fig. 5). It is obvious that an excitation of giant resonances with energies around 10-20 MeV requires bombarding energies close to one A.GeV.

Total cross sections are obtained by integrating the differential cross section with respect to impact parameter b

$$
\sigma_{\text {total }}(\pi \lambda)=2 \pi \int_{b_{\min }}^{\infty} P(b) b d b
$$

Closed expressions are found in (Winther and Alder, 1979). A problem arises with the choice of the minimal impact parameter $b_{\min }$, which is introduced in order to account for the strong absorption, occuring as soon as the colliding nuclei come within reach of their nuclear forces. In low-energy Coulomb excitation one easily avoids that problem with a bombarding energy well below the Coulomb barrier. At high energies, such 'safety' conditions cannot be fulfilled, and appropriate values for $b_{\min }$ have to be extracted by comparison to experimental data. Various parametrizations of $b_{\min }$ with regard to the nuclear system have been proposed. Most widely used are those of (Benesh et al., 1989):

$$
b_{\min }=1.34\left(A_{1}^{1 / 3}+A_{2}^{1 / 3}-0.75\left(A_{1}^{-1 / 3}+A_{2}^{-1 / 3}\right)\right)[\mathrm{fm}]
$$

and of (Kox et al., 1987):

$$
b_{\min }=1.1\left(A_{1}^{1 / 3}+A_{2}^{1 / 3}+\frac{A_{1}^{1 / 3} A_{2}^{1 / 3}}{A_{1}^{1 / 3}+A_{2}^{1 / 3}}-1.9\right)[\mathrm{fm}] .
$$

It should be noticed that interaction radii deduced from low-energy scattering data cannot be applied in a straightforward manner, since the nucleon-nucleon cross section reduces at higher energies and thus the nuclear surface might become more transparent. The assumption of a sharp cut-off at $b_{\min }$ is also questionable. Based on data (Mercier 
et al., 1986) for the one-neutron removal channel, which is the dominant Coulombinduced fragmentation channel (see below), a surface ring zone of $1.1 \pm 0.1 \mathrm{fm}$ width was deduced (Bertulani and Baur, 1988), within which nuclear and electromagnetic processes both contribute. Competitive nuclear processes will be discussed in somewhat more detail in subsection 3 .

Another theoretical concept widely used in the relativistic regime is the method of equivalent (virtual) photons, originally introduced in (Fermi, 1924) and developed independently by (Weizsäcker, 1934) and (Williams, 1934; 1935); it may also be found in (Jackson, 1975). Its main virtue is that it relates directly to real photon processes, such as photoabsorption or photon-photon scattering phenomena. This can be accomplished noticing that the electromagnetic fields (see above) are equivalent to pulses of plane polarized radiation. This is strictly true for the transversal part in the limit $\beta \rightarrow 1$. For the longitudinal part the missing magnetic field is arbitrarily added using the arguments that firstly, magnetic forces under most circumstances can be neglected and secondly, the photon intensity of the longitudinal part is suppressed by a factor $1 / \gamma^{2}$ anyway (see below).

The original ansatz of the method of equivalent photons, often refered to as the Weizsäcker-Williams (WW) method, gives photon numbers irrespective of multipolarity. Later on, equivalent photon numbers for different multipolarities were given by (Jaeckle and Pilkuhn, 1975) and (Bertulani and Baur, 1988; and references therein), where it is also shown that there is a formal equivalence between the WW method and the semiclassical perturbation approach. As far as giant dipole resonance excitations are concerned, the beauty of the WW method is that heavy ion cross sections are directly related to the photoabsorption cross section $\sigma_{\text {phot }}$, for which a large amount of data is available, via

$$
P_{\mathrm{GDR}}(b)=\int_{\mathrm{E}_{\gamma}} N\left(\mathrm{~b}, \mathrm{E}_{\gamma}\right) \sigma_{\text {phot. }}\left(\mathrm{E}_{\gamma}\right) \frac{d \mathrm{E}_{\gamma}}{\mathrm{E}_{\gamma}} .
$$

$N\left(b, E_{y}\right)$ denotes the number of equivalent photons of a frequency $E_{y} / \hbar$ and at an impact parameter $b$. The spectrum of equivalent photons for the case of multipolarity $E 1$ is of the form

$$
\mathrm{N}\left(\mathrm{b}, \mathrm{E}_{\gamma}\right)=\left(\frac{Z_{1} \alpha}{\pi}\right)^{2}\left(\frac{\xi}{\mathrm{b} \beta}\right)^{2}\left(K_{1}^{2}(\xi)+\frac{1}{\gamma_{2}} K_{\mathrm{o}}^{2}(\xi)\right)
$$

the modified Bessel functions of order zero (one) result from contributions of the longitudinal (transversal) electromagnetic pulses.

In the literature, discussions can be found on various effects, which possibly affect the results of a semiclassical calculation. Among those are quantal effects, effects due to the slight bending of the Coulomb trajectory, or effects due to the damping width of giant resonances. We also refer to (Bertulani and Baur, 1988) for detailed discussions. It turns out that these effects are barely visible within the accuracy of the data presently 
Fig. 7. Schematic calculations of total cross sections for the isovector giant dipole resonance (GDR) and isoscalar (IS) and isovector (IV) giant quadrupole resonances (GQR) in ${ }^{136} \mathrm{Xe}$. Cross sections are shown for collisions with $\mathrm{Pb}$ nuclei in a range of bombarding energies. Cross sections for higher-phonon states of the GDR are also shown (2ph, 3ph). For comparison, the geometrical cross section (G.C.) is indicated.

Fig. 8. Differential cross sections with regard to impact parameter $\mathrm{b}$ for the system $\mathrm{Pb}+\mathrm{Pb}$ at 550 A.MeV bombarding energy. Shown are cross sections for 1 to 4 phonon states (ph) of the GDR and for the isoscalar (dashed curve) and isovector (dot-dashed curve) GQR. The Rutherford cross section is also given. The data are normalized to unity at $b$ $=16 \mathrm{im}$.
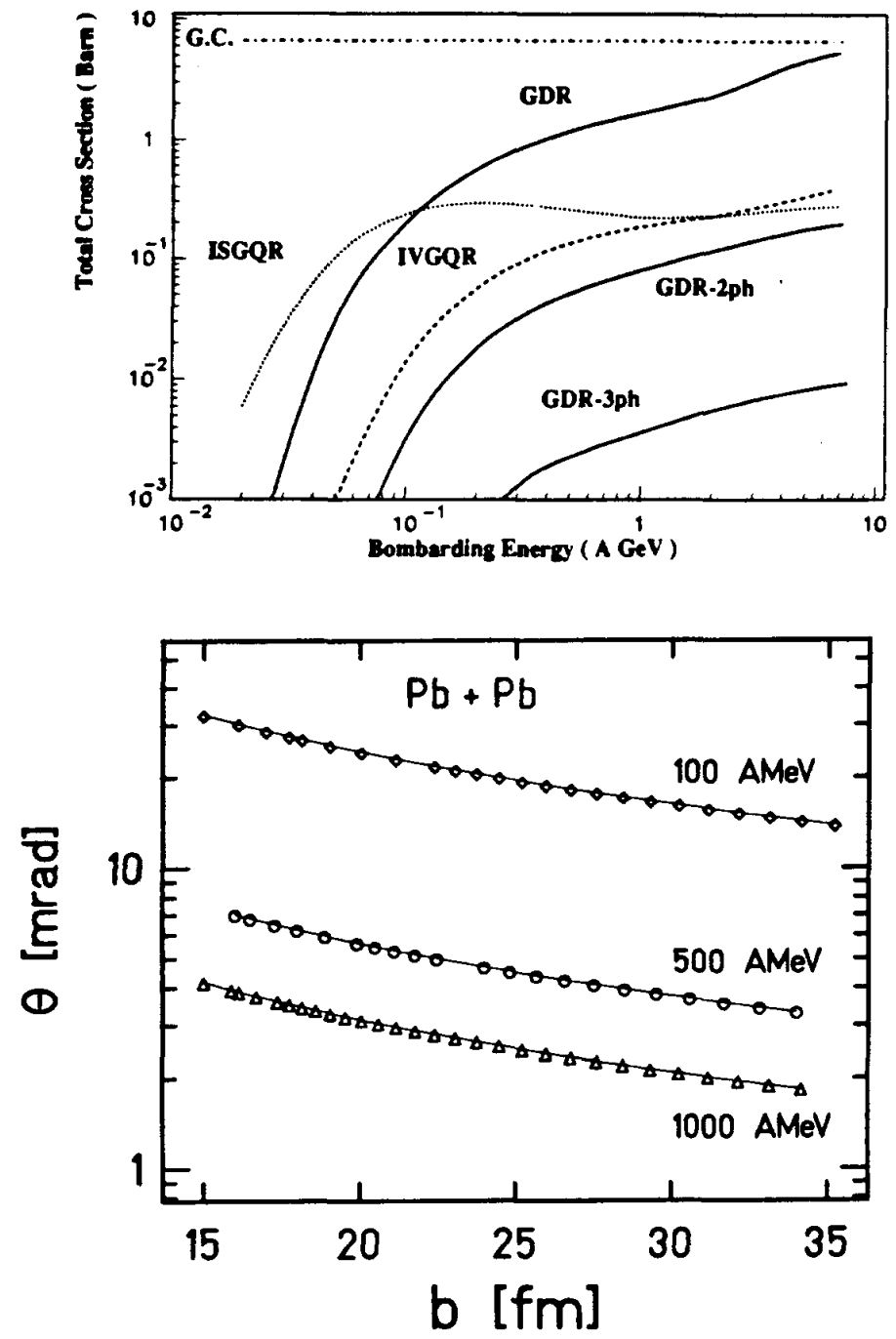

available, at least for measurements taken at sufficiently large bombarding energy ( $Z$ $500 \mathrm{~A} \cdot \mathrm{MeV})$. The validity, however, of the first order perturbation theory needs to be considered seriously. We shall return to that question.

Results of calculations based on the semi-classical first-order perturbation theory are presented in the following. It is the aim to illustrate the specific features of high-energy electromagnetic excitations and thus to provide a basic understanding for the experimental results presented in the next chapters. Most of these experiments are performed at bombarding energies close to one A.GeV and thus most emphasis is paid to that energy region. In Fig. 7, it is shown, how total cross sections for dipole and quadrupole giant resona ices evolve with bombarding energy. One observes for all giant resonances a sort of threshold behaviour which results from the adiabadicity limit. At energies $\gtrsim 200 \mathrm{~A} \cdot \mathrm{MeV}$ the dipole resonance becomes dominant, the cross section of which at the highest energies approximately exhibits a logarithmic energy dependence. At lower bombarding energies, the isoscalar giant quadrupole resonance is of similar 


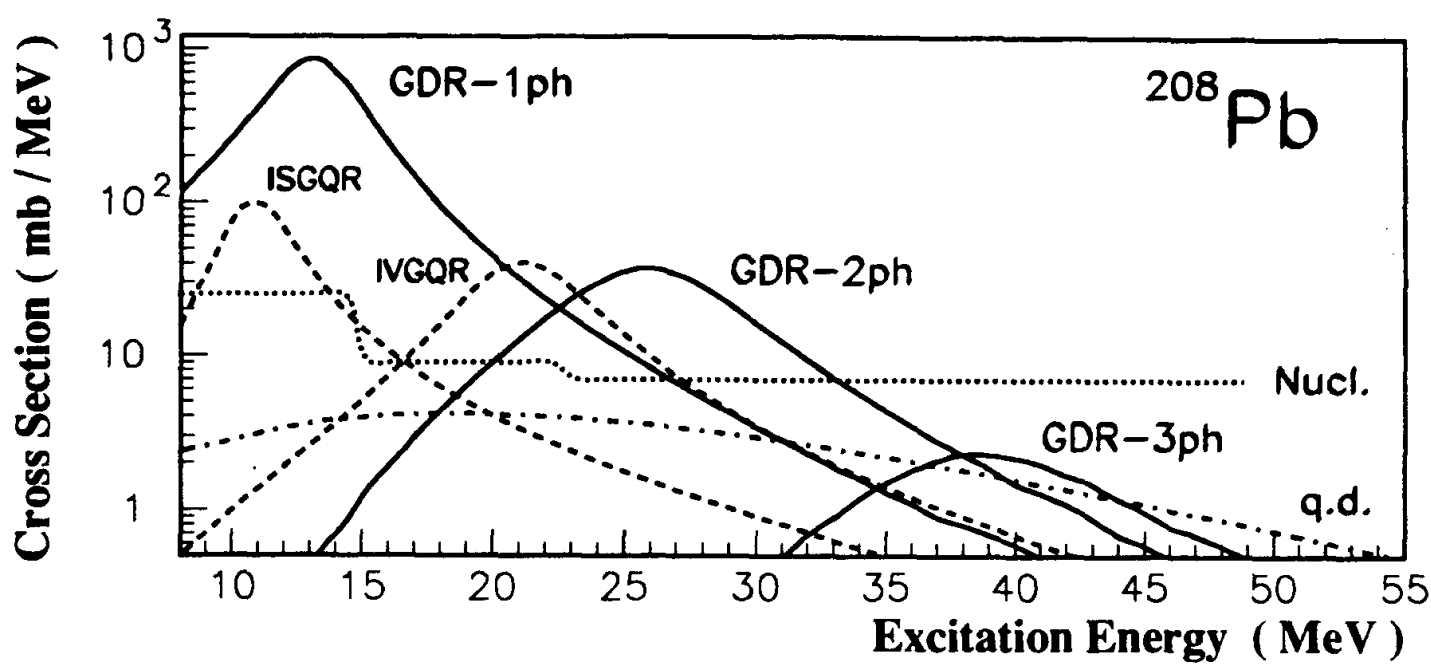

Fig. 9. Calculated differential cross sections with regard to excitation energy, integrated over impact parameter, for the electromagnetic excitation of 1 to 3 phonon states of ine GDR (solid lines) and for single-step excitations of giant quadrupole resonances (dashed lines). The notation corresponds to that of Fig. 7. Cross sections for the quasi-deuteron process are also shown (dashed-dotted line). For the strength distributions, which served as input into the calculation, see Fig. 2 and refences given there. In addition, nuclear cross sections for few-neutron removal reactions are incorporated (dotted line; see chapter 3.4). The calculations are for the excitation of ${ }^{208} \mathrm{~Pb}$ interacting with $\mathrm{U}$ at a bombarding energy of $1000 \mathrm{~A} \cdot \mathrm{MeV}$.

or even larger magnitude, because of its lower excitation energy, but also since at lower bombarding energies the E2 photon numbers - in the language of the WW method - exceeds that of the E1 photons. The summed cross section exceeds the geometrical cross section at bombarding energies $\gtrsim 10 \mathrm{~A} \cdot \mathrm{GeV}$ and thus Coulomb dissociation becomes the maior part of the total reaction cross section. Differential cross sections with regard to impacı parameter are shown in Fig. 8 for an intermediate energy of $550 \mathrm{~A} \cdot \mathrm{MeV}$. They exhibit no structures which would be characteristic of multipolarity, which thus might be difficult to disentangle in angular distribution measurements. Differential cross sections with regard to excitation energy (Fig. 9) are also evaluated. For each multipolarity, the calculated cross section distribution can be compared to the strength distribution which was used as input into the calculation. Only small distortions of the strength distribution due to the reaction dynamics are observed. It should be noted, however, that distortions might become relevant when going to lower bombarding energies as is discussed for example in (Suomijärvi et al., 1990). In all above calculations, first-order perturbation theory was applied. It is valid only in the limit that probabilities per collision are small compared to unity; this condition is not necessarily fulfilled. Excitation probabilities for giant resonances at different bombarding energies and for an impact parameter of $15 \mathrm{fm}$ are displayed in Fig. 10. For such close collisions, the condision of small probabilities obviously is not well fulfilled and at still higher bombarding energy even the unitarity limit can be violated. Large probabilities give rise to multi-step excitations, which are considered below. 
Fig. 10. Cumulative excitation probabilities for giant resonances (notation as in Fig. 7) in ${ }^{208} \mathrm{~Pb}$ at grazing impact $(b=15 \mathrm{fm})$ with $U$ ions of different bombarding energies.

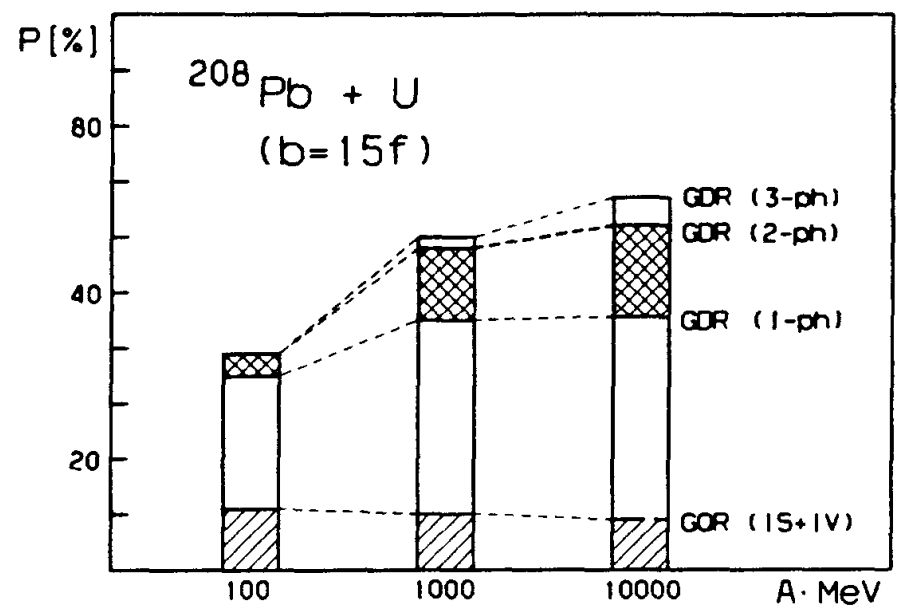

3.2 Multi-Step Excitations

As evident from Fig. 10, ii is likely that multi-step processes occur in electromagnetic heavy ion scattering. The question arises what a multiple excitation in the case of giant resonances does imply. Giant resonances are generally considered to manifest smallamplitude, harmonic density oscillations in the interior of nuclei. Multi-step excitations might thus be associated to the excitations of higher-phonon states of the harmonic oscillator as outlined in chapter 2.4. In the microscopic model, this corresponds to the independent excitation of $n$ coherent $1 \mathrm{p} 1 \mathrm{~h}$ states, which do not interact with each other. Thus one phonon is built on top of another one without interfering. This would be a very specific manifestation of Brink's hypothesis (Brink, 1955) that a giant resonance can be built on top of any excited state. A schematic model was developed (Bertulani and Baur, 1988), where it is presumed that an indefinite number of oscillator quanta can be excited in a nucleus, each one of same characteristics. The giant dipole resonance strength was concentrated into one single state at the GDR mean energy, thus ignoring damping. With these assumptions, an analytical solution can be derived (Merzbacher, 1970) for the excitation of higher phonon states. The $n$-phonon excitation probabilities $P_{n}$ obey simply a Poisson distribution,

$$
P_{n}=\frac{1}{n !} \phi^{n} e^{-\phi}
$$

where $\phi$ is the first-order perturbation probability that one phonon can be excited (to be distinguished from the probability $P_{1}$ that exactly one phonon is excited). This ansatz can easily be incorporated into the WW method or the semiclassical method. A somewhat more refined version based on the WW picture, which allows to take into account the width of the giant resonance was presented in (Llope and Braun-Munzinger, 1990). The basic idea is to calculate $P_{n}$ from above relation, but then to spread the strength for each 
phonon in excitation energy according to the strength distribution for the single phonon state as known from photoabsorption experiments. This leads to a 'folding model'. Naturally, except for distortions due to the adiabadicity as discussed above, the result is that the mean excitation energy $E_{n}$ and the width $\Gamma_{n}$ for the $n$-phonon state fulfill $E_{n}=n E_{1}$ and $\Gamma_{n}=n \Gamma_{1}$, if a lorentzian distributed strength for the single-phonon state is supposed. In Figs. 7-10 corresponding estimates for multi-phonon giant resonance states, arising from this model, are incorporated. At high energies and in heavy collisional systems, cross sections up to about 1 barn for the double GDR and up to about $100 \mathrm{mb}$ for the three-phonon GDR can be expected. Particularly from Fig. 9, it becomes evident that a clean signal at least for the two-phonon state should be observed above the 'background' arising from single-step excitations and nuclear fragmentation (see below). It is worthwhile noting that the $n$-phonon GDR cross sections show an approximate dependence

$$
\sigma_{n-\rho h} \sim\left(Z_{1} \alpha\right)^{2 n} \mathrm{~A}^{(4 / 3) n}
$$

which is derived from the strength of the electromagnetic field and the dipole sumrule, and utilizing above relation for $P_{n}$. A denotes the mass number of the excited nucleus, $Z_{1}$ the nuclear charge of the interacting partner. It is implied that favorabale experimental conditions for an observation of two- and higher phonon states are met in a system of two heavy ions.

An exact treatment of multi-step processes requires coupled channel calculations, which presently are not available. In a number of papers (Bertulani and Zelevinsky, 1993; Ponomarev et al., 1993; Typel and Baur, 1993) instead, second order perturbation theory was applied which is a straight forward extension of the first-order theory. For not too large values of $\phi$, it is a valid approximation. For near grazing collisions, which contribute most to the multi-phonon excitation (see Fig. 8), however, excitation probabilities may become large (see Fig. 10) and the validity of second-order perturbation theory becomes questionable.

In studying higher-phonon GDR states one has to consider the optimal choice of the bombarding energy. At one hand, increasing the bombarding energy yields larger cross sections, on the other hand, a higher bombarding energy gives rise to direct continuum excitations via the quasi-deuteron process and also enhances direct excitations of higher-lying resonances. In particular, the IVGQR may contribute considerably to the background underlying the two-phonon GDR. The optimal choice is that of using a bombarding energy such that the adiabatic cut-off becomes effective just above the GDR energy $E_{0}$. It was suggested to apply (Llope and Braun-Munzinger, 1992)

$$
E_{0}+\Gamma \lesssim \frac{\hbar \beta \gamma}{b_{\min }} \lesssim 2 E_{o}-\Gamma \text {. }
$$

This leads to values of 500-700 A.MeV bombarding energy for the heaviest systems and to slightly lower values for lighter systems. 


\subsection{Nuclear Fragmentation}

As already indicated, nuclear fragmentation might be a source of physical background in multi-phonon GDR studies, since it involves reaction channels identical to those observed from electromagnetic dissociation. For heavy systems the few-neutron removal channels have to be considered in particular, since for example the DGDR is expected to decay by emission of 2-4 neutrons in most cases (see Fig. 2). Numerous target fragmentation studies have been performed using projectiles ranging from protons up to the heaviest elements in a wide range of bombarding energies. As a result, the concepts of factorization and limiting fragmentation emerged. Factorization means that the production cross section for a specific isotope $\sigma_{\mathrm{F}}$ in heavy ion collisions relates to the production cross section in collisions with protons by a simple scaling factor $S_{H 1}$ which is independent from the produced fragment (Hill et al., 1988, and references therein):

$$
\sigma_{F}(H I+T)=S_{H I} \sigma_{F}(\text { proton }+T)
$$

$\mathrm{T}$ denotes the target nucleus, $\mathrm{HI}$ the heavy ion beam while targetlike fragments $\mathrm{F}$ are considered. The scaling factor can be deduced from fragments whose production is not influenced by electromagnetic processes, for example from charge-removal channels in heavy ions, and thus the nuclear contribution to the few-neutron removal channels of interest can be obtained using proton spallation data. Limiting fragmentation means that production cross sections are independent of the bombarding energy. The validity of limiting fragmentation was strictly shown only for proton energies above $\sim 10 \mathrm{GeV}$, but cross sections for few nucleon removal channels were found to remain essentially constant at proton energies $\gtrsim 200$ A.MeV (Kaufman et al., 1976, 1980a, 1980b). Using the above scaling, nuclear few-neutron removal cross sections were extracted for ${ }^{197} \mathrm{Au}$ bombarded with various ions. A compilation of data is provided in (Aumann et al., 1993a; see references therein) which is shown in Fig. 11 and which is representative for heavy nuclei. For the heaviest projectiles, cross sections for the one-neutron removal channels amount to $100-200 \mathrm{mb}$, for the two- and three-neutron removal channel to about $50 \mathrm{mb}$. In order to compare with the corresponding electromagnetic cross sections, we incorporate such values into Fig. 9, where the nuclear cross sections are spread homogenously between the relevant thresholds for neutron emission. Obviously, this nuclear background is small in the case of one- and two-phonon GDR, but cannot be neglected for higher-phonon states. It is evident from Fig. 11 that the nuclear target fragmentation cross sections obey an approximate $A_{p}^{1 / 3}$ dependence, because the fewnucleon removal reactions occur in a rather narrow surface zone. Such a dependence is inferred from calculations in the Glauber model (Benesh et al., 1989) or from intranuclear cascade calculations (Aumann et al., 1993a), on the basis of which also reliable cross section estimates may be derived. In most of the publications, it is assumed that nuclear and electromagnetic fragmentation cross sections add incoherently, and interference terms are neglected. Interferences through the isovector giant dipole resonance are of most interest here. Estimates were given in (Benesh et al., 1989) for the oneneutron removal channel in ${ }^{197} \mathrm{Au}$ bombarded with heavy ions, and values of $\leq 20 \mathrm{mb}$ are quoted, corresponding to less than 0.5 per cent of the expected electromagnetic dissociation cross section. To summarize, competing nuclear fragmentation processes 
Fig. 11. Nuclear in to 3n-removal cross sections for ${ }^{197} \mathrm{Au}$ targets as a function of projectile nuclear mass, $A_{\text {proj. }}$. The full dots represent experimentally determined cross sections, the compilation of data is due to (Aumann et al., 1993a). Open circles result from an intra-nuclear cascade calculation, the solid lines represent an interpolating $A_{p r o j}^{1 / 3}$ dependence. The dotted lines indicate an estimate of errors attributed to calculated nuclear cross sections.

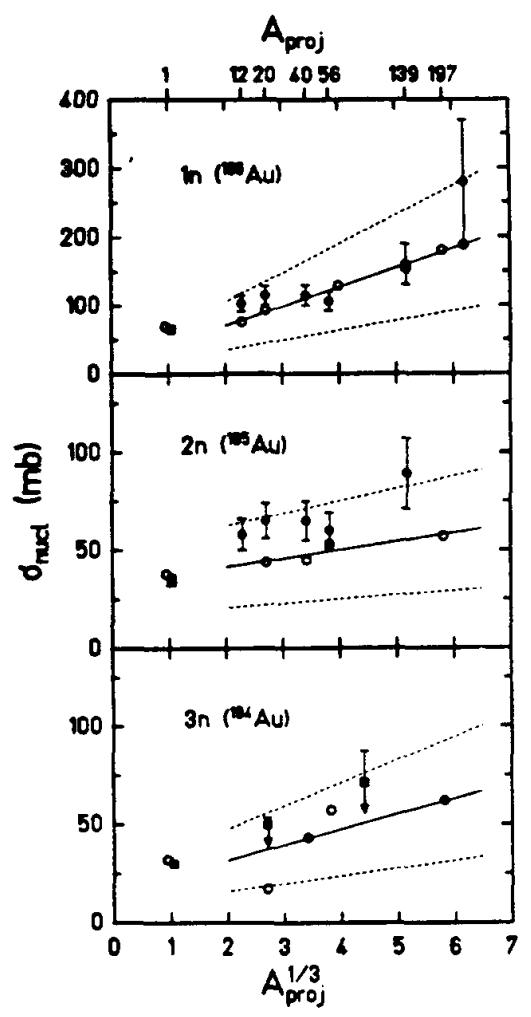

are expected to contribute only small cross sections in heavy systems as far as reaction channels relevant for the two-phonon GDR are concerned, but may form a considerable background for higher-phonon states. The nuclear and electromagnetic fragmentation cross sections, however, depend in a characteristic manner on charge and mass of the interacting partner, and thus systematic measurements should allow to disentangle their respective contributions.

\section{EXPERIMENTAL STUDIES OF THE DOUBLE GIANT DIPOLE RESONANCE}

According to the discussions in the previous chapters, the following scenario emerges, which is relevant for experimental studies of the double isovector giant dipole resonance. Collisional systems involving two partners of highest mass and charge yield the largest cross section, the most suitable bombarding energies are slightly below $1 \mathrm{~A} . \mathrm{GeV}$. The double giant dipole resonance should preferentially decay via the em ssion of two to four neutrons (see Fig. 3), a direct double $\gamma$-decay branch of the ord $\epsilon_{\text {: }}$ of $\sim 10^{-4}$ is expected for example for the case ${ }^{208} \mathrm{~Pb}$. Based on this information, different experiments aiming at a study of the electromagnetic DGDR excitation were designed and carried out at the heavy ion synchrotron facility SIS at GSI, Darmstadt. Short reviews on the GSI data were presented earlier (Emling, 1993a; 1993b). Experiments concentrate on ${ }^{208} \mathrm{~Pb}$, but results were also obtained for ${ }^{136} \mathrm{Xe}$ and ${ }^{197} \mathrm{Au}$. Similar experiments, but at considerably 
lower energy were performed meanwhile at the GANIL facility, Caen, and for light ions at much higher energy at the AGS facility, Brookhaven.

The conventional approach in studying inelastic scattering is that of using high resolution magnetic spectrometers. An application of this technique is hardly feasible at (near) relativistic bombarding energies, since resolutions of the order of one $\mathrm{MeV}$ - in comparison to total kinetic energies of the order of $100 \mathrm{GeV}$ - are required. Therefore, a resolving power of the order of $10^{5}$ with regard to magnetic rigidity would be to achieved. Moreover, the relevant laboratory scattering angles are of the order of a few milliradians and thus beams of extraordinary emittance are needed. Ray-tracing techniques also can hardly be applied because of angular straggling effects in the detectors. For such reasons, new instrumental methods had to be developed which will be described briefly.

Different experimental techniques were applied: Activation measurements of inclusive type, fully exclusive measurements, and observations of the weak direct $\gamma$ decay. A short description of these experiments is given in subsections 1-3 including their results. The information on the DGDR, available from a physically different process, i.e. the pion double charge exchange reaction, is summarized in subsection 4. Evidence for twophonon states of the isoscalar giant quadrupole resonance was reported recently from scattering data of lighter ions at relatively low energy, which will be commented on in subsection 5 .

\subsection{Activation Measurements}

The strongest decay channel of the giant dipole resonance in heavy nuclei, as known from photoabsortion measurements, is the one-neutron decay. Consequently, the heavy ion induced electromagnetic dissociation process was studied extensively by measuring the $1 \mathrm{n}$ removal cross section in target activation measurements. The experimental procedure is rather simple and consists of irradiating the target $(Z, A)$ with energetic heavy ion beams and observing the $\gamma$-ray activity following $\beta$ decay of the product nucleus $(Z$, A-1). ${ }^{197} \mathrm{Au}$ targets suit particularly well for such studies because of the favorable decay characteristics of ${ }^{196} \mathrm{Au}$. Fig. 12 (left panel) summarizes data obtained for this nucleus (Aumann et al., 1993b). It exemplifies the basic features of the heavy ion induced electromagnetic dissociation, for instance the approximate $Z_{\text {oroj }}^{2}$ dependence and large cross sections up to several barns. Nuclear contributions, derived from procedures discussed in chapter 3.3 and included in Fig. 12, are obviously small.

It was pointed out (Aumann et al., 1993a), that information on multi-step excitations, in particular for the DGDR, can be derived from analogous measurements of the two- and three-neutron removal reactions, since the DGDR should decay preferentially through these channels. They performed a measurement irradiating a ${ }^{197} \mathrm{Au}$ target with ${ }^{197} \mathrm{Au}$ and ${ }^{209} \mathrm{Bi}$ beams of $1.0 \mathrm{~A} \cdot \mathrm{GeV}$ energy at the SIS facility and determined the total cross sections for $1 \mathrm{n}, 2 \mathrm{n}$ and $3 \mathrm{n}$ removal. The measured cross sections, after correcting for nuclear contributions, were then compared to calculations for the electromagnetic excitation-dissociation process taking into account E1 and E2 excitations and, in particular, the two-phonon E1 excitation employing the folding model (see section 3.2). 

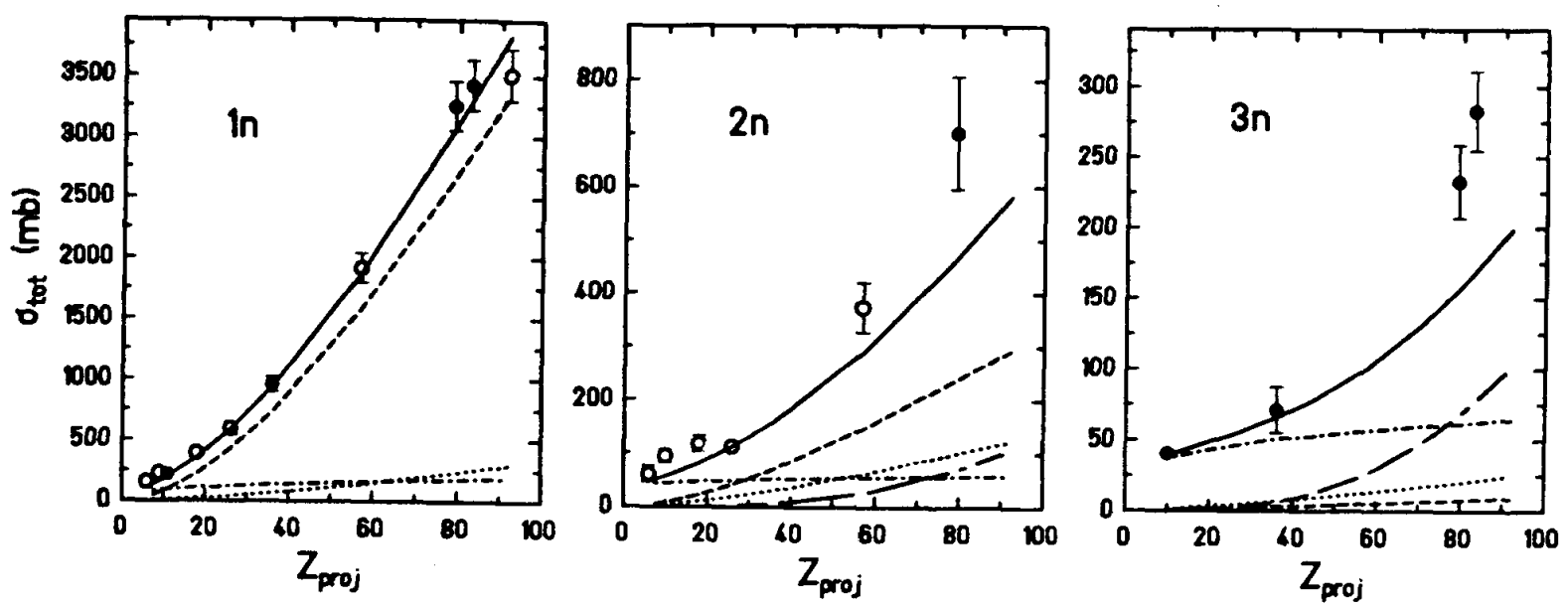

Fig. 12. Experimental $1 n$ to $3 n$ removal cross sections from ${ }^{197} \mathrm{Au}$ bombarded with heavy ions at $1 \mathrm{~A} \cdot \mathrm{GeV}$ (data taken at different incident energies have been scaled to $1 \mathrm{~A} \cdot \mathrm{GeV}$ using the energy dependence of the WW theory). The full curve denotes a theoretical calculation including nuclear cross sections (dot-dashed curve), and electromagnetic cross sections for the excitation of the GDR (dashed curve), the sum of isoscalar and isovector GQR (dotted curve) and the twophonon GDR (long dashed curve) (Aumann et al., 1993b).

Their experimental results and calculations are shown in Fig. 12. It becomes evident that the cross sections for the $2 n$ and $3 n$ removal channel cannot be explained by electromagnetic single-step excitations only. But even after adding the calculated DGDR cross section, the calculation underestimates the experimental $2 n$ and $3 n$ cross sections considerably, while it has no effect on the $1 n$ cross section. The summed $2 n+3 n$ cross sections exceed the calculation by about $300 \mathrm{mb}$. In order to bring the calculation in line with this value, the calculated cross section for the DGDR would have to be raised by a factor of 2 at least.

Of course, from such inclusive data one cannot deduce resonance parameters such as width and excitation energy of the DGDR. Also the cross sections for the DGDR are derived only in an indirect way, since the procedure heavily relies on the accuracy of the theoretical description of the electromagnetic excitation process and of the subsequent de-excitation. On the other hand, it was shown (Aumann et al., 1993a; 1993b) that, in changing free parameters of the calculation such as the $b_{\min }$ parameter, which is of most influence, one cannot achieve a consistent description for all measured neutron-removal channels.

\subsection{Exclusive Measurements and Neutron Decay}

Exclusive measurements aiming to find evidence for the double giant dipole resonance were carried out by the LAND collaboration at GSI, Darmstadt. Their experimental method allows to determine differential cross sections with regard to excitation energy, 


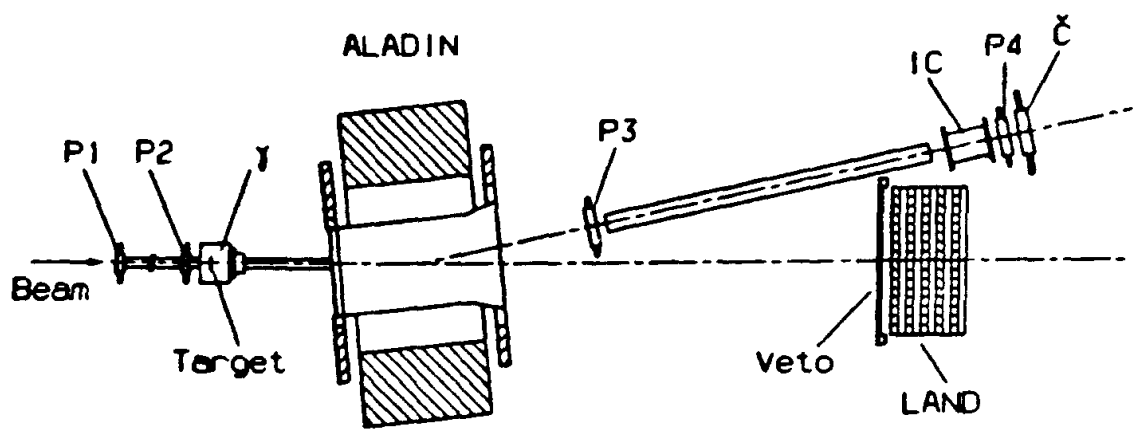

Fig. 13. Experimental set-up used by the LAND collaboration (Schmidt et al., 1993). Shown are beam and fragment detectors (P1-P4, IC, C), the dipole magnet (ALADIN), the $\gamma$-detector array ( $\gamma$ ) and the neutron detector (LAND, Veto).

i.e. to obtain the strength distributions, to measure the neutron decay patterns and, in addition, to check the reaction dynamics of the process of electromagnetic excitation. The method relies on projectile excitation rather than target excitation, which is of no principal restriction since the SIS facility is capable of delivering beams of almost any stable isotope and even of unstable nuclei. The main advantage of employing projectile dissociation is the fact, that the projectile-like fragments and ejectiles are kinematically focussed into a narrow forward cone. Thus, the relevant solid angle can easily be subtended and detection systems with efficiencies close to unity can be built. A schematic view of the experimental set-up is given in Fig. 13. The trajectories of the incoming projectile and that of the heavy fragment emerging from the target are traced by means of position-sensitive multiwire-gas counters and plastic detectors, which also deliver accurate time-of-flight information. The nuclear charge of the heavy fragment is determined by means of an ionization chamber and a Cherenkov detector. The fragments (or projectiles) are deflected in a dipole magnet in order to allow an unperturbed measurement of neutrons around $0^{\circ}$ with regard to the beam axis. The dipole magnet has a sufficiently wide gap of $1.6 \mathrm{~m} \times 0.48 \mathrm{~m}$ to allow for a free passage of neutrons emitted from the excited fragments. The neutrons impinge on a detector of $2 \mathrm{~m} \times 2 \mathrm{~m}$ active area after a travel distance of about $10 \mathrm{~m}$. This Large Area Neutron Detector (LAND) (Blaich et al., 1992) is a special development for neutrons of intermediate energy. It utilises a technique developed for neutral particle calorimeters, i.e. the conversion into charged particle showers in Fe layers with interspersed organic scintillation detectors. The special design, however, allows for accurate position and time-of-flight measurements of inci- 
dent neutrons, and in addition, has a certain capability of resolving multiple hits. This was achieved by modularizing the detector into 200 independently operating elements. Neutrons are distinguished from incident charged particles by means of a thin plastic wall in front of the detector. The detection efficiency increases with neutron energy and ranges from $50 \%$ at $100 \mathrm{MeV}$ to $95 \%$ at $1 \mathrm{GeV}$ or higher neutron energies.

Differential cross sections with respect to excitation energy of the projectile are obtained by utilising the lorentz-invariant quantity (invariant mass)

$$
M^{2}=\left(m_{\text {proj }}+E^{\star}\right)^{2}=\left(\sum_{j} P_{j}\right)^{2}
$$

where $P_{i}$ denotes the 4-momenta of the fragment and the neutrons, $m_{\text {proj }}$ and $E^{\star}$ denote the groundstate mass and excitation energy of the projectile, respectively. The residual fragment is not necessarily in its goundstate after neutron emission, and its remaining excitation energy will be released by $\gamma$ de-excitation cascades. Above relation can conveniently be rewritten as

$$
M=\left(\sum_{j} m_{j}^{2}+\sum_{i \neq j} \gamma_{i} \gamma_{j} m_{i} m_{i}\left(1-\beta_{j} \beta_{j} \cos \left(\vartheta_{i j}\right)\right)\right)^{1 / 2}+E_{F}^{*}
$$

involving groundstate masses $m_{i}$, velocities $\beta_{i}$ and relative angles $\vartheta_{11}$ between neutrons and between neutrons and fragment. The residual excitation energy $E_{F}^{*}$ of the fragment can be identified with the total $\gamma$-ray energy released by the fragment, if evaluated in the projectile rest frame; small recoil terms are neglected. With the apparatus shown in Fig. 13 the excitation energy $E^{\star}$ can be extracted with an accuracy of typically $1 \mathrm{MeV}$. The total $\gamma$-ray energy, measured in an array of $\mathrm{BaF}_{2}$ scintillation counters surrounding the target (see Fig. 13), however, is less precise, resulting in an overall resolution of $\sigma_{\mathrm{E}^{*}}$ $\approx 2 \mathrm{MeV}$.

A necessary prerequisite for the method of invariant mass reconstruction is that the fragment and ejectiles emerging from the disintegrating projectile are well separated in momentum space with regard to particles emitted by any other source, e.g. from the target. This condition is well fulfilled in peripheral energetic heavy ion collisions, an illustration will be given below. This occurs because of the low momentum transfer (see chapter 3.1) which is negligible in comparison to the initial projectile momentum. For emitted $\gamma$ rays this is only partially true, since the Lorentz boost is less effective than for massive particles. This problem can partially be circumvented with a $\gamma$ detector covering only the forward cone, into which $\gamma$ rays from projectile fragments are boosted, while $\gamma$ rays from target fragments subtend the full laboratory solid angle. Arranging the $\gamma$ detector in such a way, it was estimated that for the experiments discussed here, simultaneous target excitation, if occurring, would contribute $2 \mathrm{MeV}$ at most to the reconstructed excitation energy and such effects, therefore, are within the experimental resolution. Experiments of this type were performed by the LAND collaboration for the 

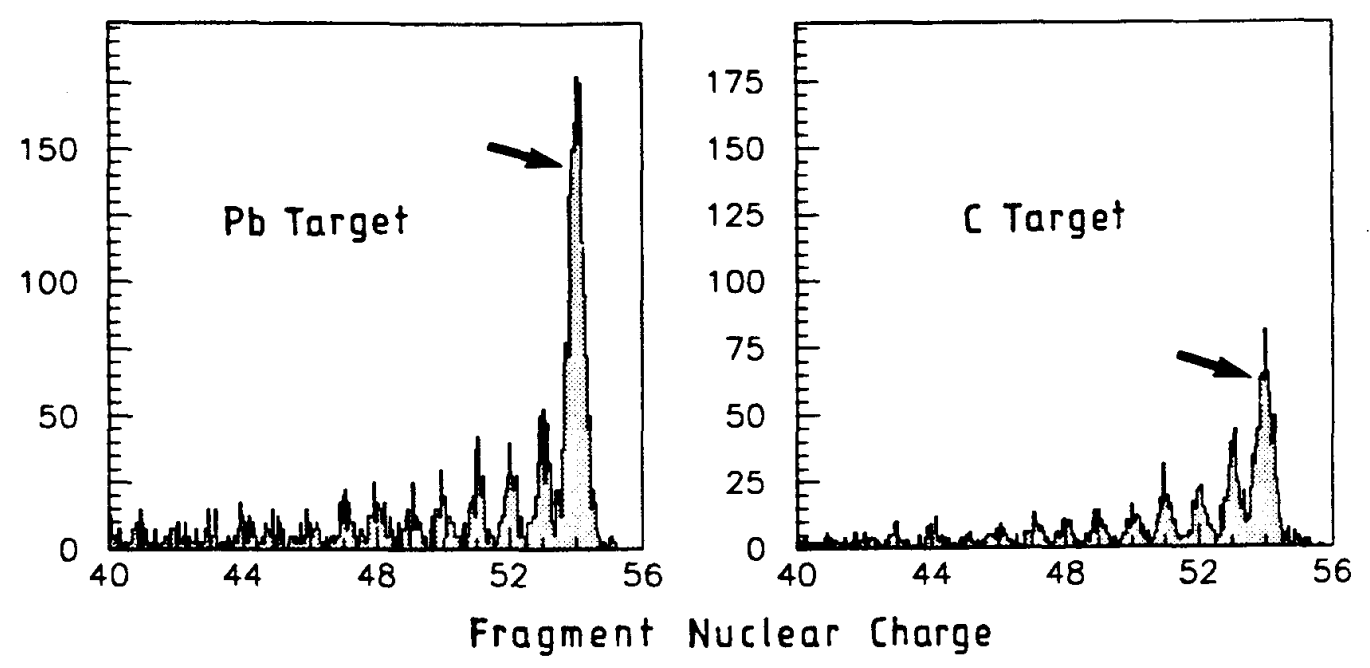

Fig. 14. Nuclear charge distribuıion of projectile $\left({ }^{136} \mathrm{Xe}, 700 \mathrm{~A} \cdot \mathrm{MeV}\right)$ fragments, observed with a $\mathrm{Pb}$ target (left) and a $\mathrm{C}$ target (right) (Spies, 1992). The pure neutron-removal reactions are indicated by arrows.

semi-magic nucleus ${ }_{54}^{16} X e$ (Schmidt et al., 1993) and the double-magic nucleus ${ }_{82}^{208 b}$ (Wajda et al., 1993) at bombarding energies of 700 and $650 \mathrm{~A} \cdot \mathrm{MeV}$, respectively. With these projectiles heavy targets such as $\mathrm{Pb}$ or $\mathrm{U}$ were bombarded for studies of the electromagnetic processes, and light targets were used in order to determine background from nuclear interactions. In Fig. 14 the fragment nuclear charge distribution for ${ }^{136} \mathrm{Xe}$ projectiles on $\mathrm{Pb}$ und $\mathrm{C}$ targets are displayed (Spies, 1992), which were measured under the constraint that a fast neutron was hitting the neutron detector. For the $C$ target a smooth trend in the nuclear charge distribution is found, including the pure neutronremoval channels $(Z=54)$. In contrast, when using the $\mathrm{Pb}$ target, the pure neutron removal channels exhibit a large excess yield in comparison to charge-removal channels. This is a clear manifestation of inelastic electromagnetic excitation followed by neutron decay. Selecting the pure neutron-removal reactions, one can illuminate the reaction dynamics of the electromagnetic process. The transverse momentum distribution of such fragments for the ${ }^{208} \mathrm{~Pb}+\mathrm{Pb}$ system at $650 \mathrm{~A} \cdot \mathrm{MeV}$ is shown in Fig. 15, and reflects an extremely low momentum transfer. In fact, the width of the distribution is basically determined by instrumental effects such as straggling in the target or detector material. The exclusive measurements thus provide convincing evidence that in heavy ion systems the pure neutron-removal channels indeed arise from a very soft excitation mode, whose reaction dynamics is consistent with what is expected for inelastic electromagnetic excitations into the giant resonance domain. For the $1 \mathrm{n}$ to $3 \mathrm{n}$ removal channels the invariant mass reconstruction was performed for the case of ${ }^{136} \mathrm{Xe}$ projectile excitation (Schmidt et al., 1993). The resulting cross section, differential in excitation energy, is given in Fig. 16 for a $\mathrm{C}$ target and a $\mathrm{Pb}$ target. A comparison of the two spectra immediately proofs that nuclear contributions are small. By scaling the cross section measured with the $C$ target following methods discussed in chapter 3.3 , it was concluded that nuclear interactions contribute only $\sim 100 \mathrm{mb}$ to the total cross section measured with the $\mathrm{Pb}$ target, which amounts to 1.85 (10) barn. Consequently, the spec- 
Fig. 15. Fragment transverse momentum distribution observed for the pure neutron removal channels in the system ${ }^{208} \mathrm{~Pb}(650 \mathrm{~A} \cdot \mathrm{MeV})+\mathrm{Pb}$ (Stroth and Wajda, 1993). Instrumental effects are not deconvoluted, contributions from nuclear reactions are not subtracted. The arrow indicates the transverse momentum expected for grazing collisions.

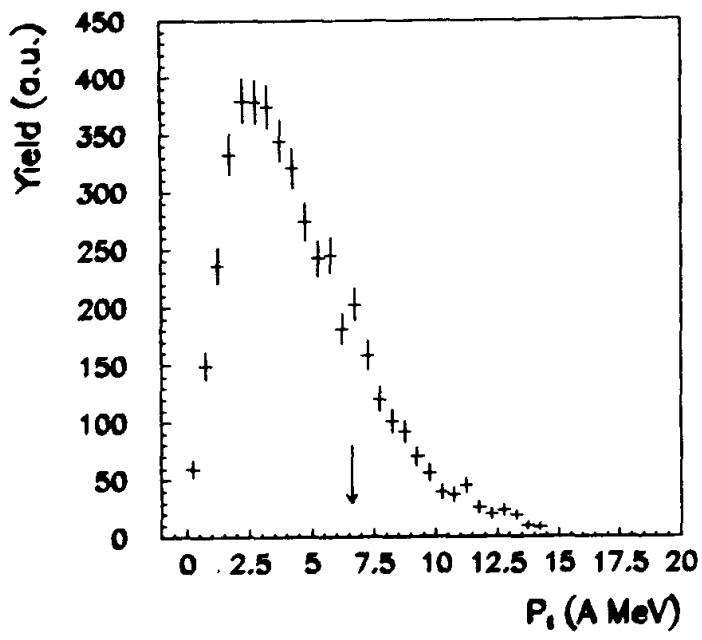

trum obtained for the $\mathrm{Pb}$ target was analyzed in terms of electromagnetic excitations. Using the method of equivalent photons, but in a formulation which is fully equivalent to the semiclassical straight line approximation in first-order perturbation theory, the spectrum below $25 \mathrm{MeV}$ excitation energy can be well reproduced, if excitations of the isovector GDR and isoscalar and isovector GQR's are taken into account (compare Fig. 16). Higher multipolarities were estimated to be negligible. Lorentzian parametrizations as obtained from photoabsorption measurements for the GDR and from data systematics in the case of giant quadrupole resonances entered as input into this calculation. Parameters describing the giant resonances, i.e. their shape and transition strength, essentially need not to be modified, except for the transition strength of the GDR, which had to be reduced by $35(4) \%$ with regard to the photoabsorption data. The total cross section comprised in the GDR and GQR's amounts to $1460(80) \mathrm{mb}$.

Centered around $28 \mathrm{MeV}$, a prominent additional structure is observed, which was identified as the double excitation of the giant dipole resonance. The corresponding part of the excitation energy spectrum after subtracting the calculated contributions from single step excitations of GDR and GQR's is also shown in Fig. 16. For this resonance a mean energy of 28.3(7) MeV, a width (FWHM) of $6.3(1.6) \mathrm{MeV}$ and an integrated cross section of $215(50) \mathrm{mb}$ was deduced. Due to the adiabaticity limit (see chapter 2), which becomes effective around $\sim 20 \mathrm{MeV}$ excitation energy, it could be excluded that a single step excitation can account for the observed cross section. The most likely two-step process is that of a double excitation of the GDR, two-step processes involving other multipolarities were calculated to be negligible. Observing that in the respective energy region almost no cross section is found when using the $C$ target, effects due to nuclear excitations were also excluded. 

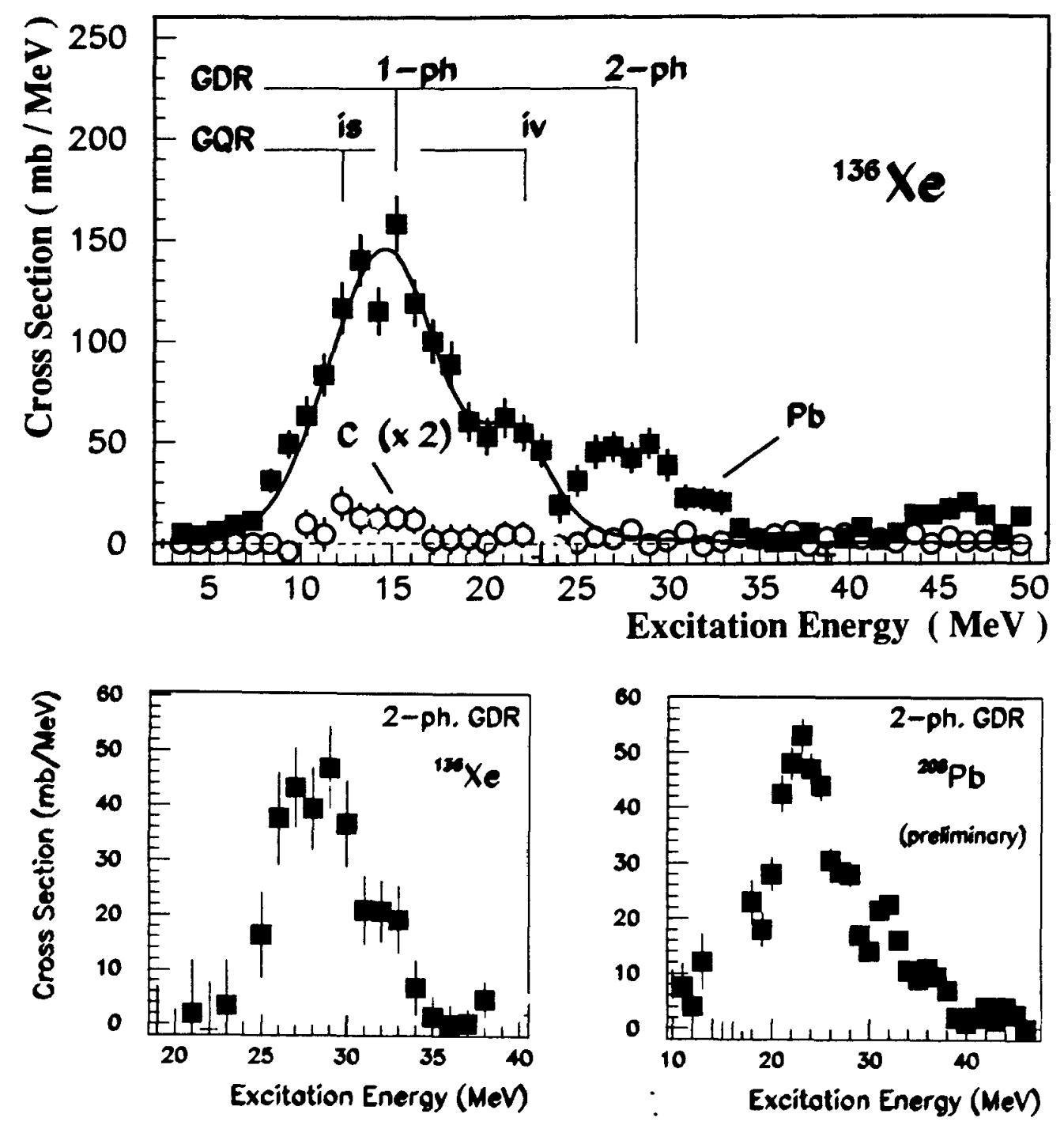

Fig. 16. (upper part) Spectrum of ${ }^{136} \mathrm{Xe}$ (projectile) excitation on a $\mathrm{Pb}$ taryet (squares) and on a $C$ target (circles); the latter one is scaled by a factor two. The resonance energies for the oneand two-phonon giant dipole resonance (GDR-1ph,2ph) and for the isoscalar (is) and isovector (iv) quadrupole resonances(GQR) are indicated. The solid curve reflects the result of a first-order semiclassical calculation for the Pb target (Schmidt et al., 1993).

(lower left part) Same as upper part, however, after subtracting the first-order calculation (solid curve in upr 2 r part) from the spectrum measured with the $\mathrm{Pb}$ target and displayed in the energy range reli , ant for the DGDR.

(lower right part) Same as left part, however, for the excitation of ${ }^{208} \mathrm{~Pb}(650 \mathrm{~A} \cdot \mathrm{MeV})$ on a $\mathrm{Pb}$ target (Wajda et al., 1993). The same procedure of subtracting single step excitations was applied as in the case of ${ }^{136} \mathrm{Xe}$. In addition, contributions from nuclear interactions determined by means of a measurement with a $C$ target are subtracted. 
An analogous experiment was performed by the LAND collaboration for the magic nucleus ${ }^{208} \mathrm{~Pb}$ and a similar analysis was performed. Data were taken for ${ }^{208} \mathrm{~Pb}$ projectiles $(650 \mathrm{~A} \cdot \mathrm{MeV})$ and a number of targets ranging from $\mathrm{C}$ to $\mathrm{U}$. Only preliminary results were published (Wajda et al., 1993). The excitation energy spectrum for the DGDR in ${ }^{208} \mathrm{~Pb}$ is shown in Fig. 16. Again, nuclear contributions as determined from a measurement with a C target were subtracted as well as the contribution from calculated single step electromagnetic excitations. For that purpose, the same procedure was applied as was described above for the case of ${ }^{136} \mathrm{Xe}$. The preliminary values for the double giant dipole resonance in ${ }^{208} \mathrm{~Pb}$ are a mean energy of 24.(1.5) MeV, a width (FWHM) of 6.3 (1) MeV and a cross section of $350(60) \mathrm{mb}$. The implications of the experimental results for the double giant dipole resonance in ${ }^{136} \mathrm{Xe}$ and ${ }^{208} \mathrm{~Pb}$ will be discussed in chapter 5 .

In the spectra shown in Fig. 16, additional cross section is observed around an excitation energy, which is expected for 3-phonon GDR states, and is of the order of magnitude expected for such a state. For reasons discussed in (Schmidt et al; 1993), however, such an assignment yet remains to be firmly established.

As a side remark, it is worth mentioning that the data of the LAND collaboration were obtained with beam intensities of typically $10^{4}$ particles per second only, limited by the capability of the data aquisition system. Applications with secondary radioactive beams of low intensity are thus feasible giving access to giant resonance studies over a wide range in isospin.

Neutron decay studies for the GDR were performed in the past aiming on an identification of pre-equilibrium components (Alarcon et al., 1991). In heavy nuclei, however, statistical decay was found to be predominant, possibly with direct decay compunents of less than $20 \%$. The question can be raised, if the decay of the two-phonon GDR is a also governed by the coupling to compound nucleus states or if signatures of preequilibrium decays show up. Having the hydrodynamical picture in mind, the neutron excess at the turning point of the collective neutron-proton vibration should increase with phonon number. It could be imagined that the increased amplitude of the multiphonon states thus leads to an enhanced pre-equilibrium emission of neutrons or even to emission of neutron clusters from the nuclear surface. Studies of that type for the two-phonon GDR in ${ }^{136} \mathrm{Xe}$ were performed (Schmidt, 1991). As an example, Fig. 17 displays the distribution in neutron kinetic energy, analyzed in the projectile rest frame, for the $1 \mathrm{n}$ and $2 \mathrm{n}$ emission channels. The $1 \mathrm{n}$-removal channel basically spreads in a range of excitation energies between 8-18 MeV and thus covers single step excitations of the GDR with smaller contributions from the giant quadrupole resonances. The $2 n$ removal channel comprises excitation energies in an interval of $\sim 18-30 \mathrm{MeV}$ and thus contains the largest fraction of the DGDR cross section. Below $10 \mathrm{MeV}$ both spectra are well fitted by Maxwell distributions and thus exhibit basically statistical features. A component of energetic neutrons above $\sim 10 \mathrm{MeV}$ is nevertheless observed in both spectra with an intensity of about $10 \%$. For the $1 \mathrm{n}$ channel, this observation is in qualitative agreement to corresponding results deduced from neutron decay spectra after photoabsorption (Alarcon et al., 1991). Since the spectra for the $1 \mathrm{n}$ and $2 \mathrm{n}$ channel essentially show identical features, an enhanced non-statistical component in the decay of the DGDR cannot be concluded. A more quantitative analysis and a better statistical accuracy, however, seems to be required. 

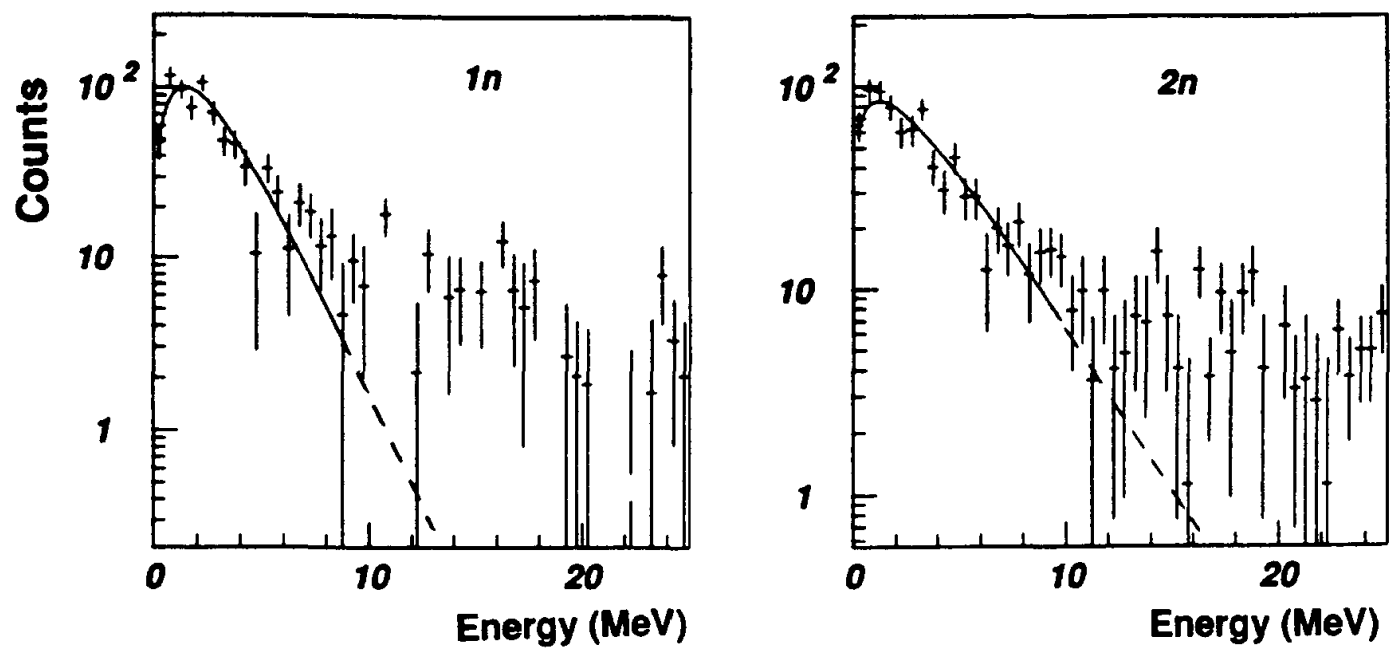

Fig. 17. (left) Spectrum of neutron kinetic energies in the projectile rest frame observed in the $\mathrm{Pb}\left({ }^{136} \mathrm{Xe},{ }^{135} \mathrm{Xe}+1 \mathrm{n}\right)$ reaction at $700 \mathrm{~A} \cdot \mathrm{MeV}$ bombarding energy (Schmidt, 1991). The experimental response function is not deconvoluted. The solid line represents a Maxwell distribution.

(right) same as left panel, however, for the $\mathrm{Pb}\left({ }^{136} \mathrm{Xe},{ }^{134} \mathrm{Xe}+2 \mathrm{n}\right)$ reaction.

The E814 collaboration searched for multi-phonon giant resonances in light nuclei (Barrette et al., 199.0; Sonnadara et al., 1993). The experimental concept is rather similar to that discussed above, i.e. projectile excitation is considered and a kinematically complete measurement is performed for projectile fragments and ejectiles. Since giant resonances in light nuclei decay as well by charged particle emission, experimental provisions were taken for such decay channels. The experiments were performed at the AGS facility, Brookhaven, with ${ }^{28} \mathrm{Si}$ beams of $14.6 \mathrm{~A} \cdot \mathrm{GeV}$ energy on $\mathrm{Al}, \mathrm{Sn}$ and $\mathrm{Pb}$ targets. Evidence for two-phonon giant resonances was not observed. The expected experimental signal, however, is very weak, we estimate cross sections of about $5 \mathrm{mb}$ for the DGDR with the $\mathrm{Pb}$ target. Processes of nuclear interactions and direct continuum excitations leading to the same reaction channels are likely to obscure the small contribution from the DGDR decay.

\section{$4.3 \gamma$-Decay Studies}

The direct $\gamma$ decay of the GDR is a weak branch where a $\gamma$ transition into the groundstate of the nucleus occurs without accompanying particle emission. This branch was measured for the GDR in ${ }^{208} \mathrm{~Pb}$ with a relative Jecay probability $\Gamma_{y} / \Gamma_{\text {tatal }}=0.017$ (Beene et al., 1990). Assuming a DGDR cross section ficically a few hundred $\mathrm{mb}$, one thus expects the double photen decay of the DGDR back to groundstate via the GDR as intermediate state with a cross section of the order of $0.1 \mathrm{mb}$.

A successful observation of this double- $\gamma$ decay from the DGDR in ${ }^{208} \mathrm{~Pb}$ was reported (Ritman et al., 1993a; Kühn, 1993). The DGDR was excited by bombarding a ${ }^{208} \mathrm{~Pb}$ target with ${ }^{209} \mathrm{Bi}$ beams of $1 \mathrm{~A} \cdot \mathrm{GeV}$ energy at the SIS of GSI, Darmstadt. Two $\gamma$ rays in coincidence were observed using the detector system TAPS (two-arm photon spectrometer; 
Fig. 18. Sum energy $E_{1}+E_{2}$ of coincident photon pairs from ${ }^{200} \mathrm{~Pb}$ excited with ${ }^{209} \mathrm{Bi}$ projectiles (1 A.GeV). The structure centered around $26 \mathrm{MeV}$ is assigned to the double $\gamma$ decay of the DGDR. The experimental spectrum is reproduced by a sum of a lorentzian and two exponential distributions (solid line), the latter ones (dashed line) are used to describe statistical $\gamma$ decays and background (Ritmann, 1993a). The inset shows a lorentzian distribution fitted to the difference between data and background.

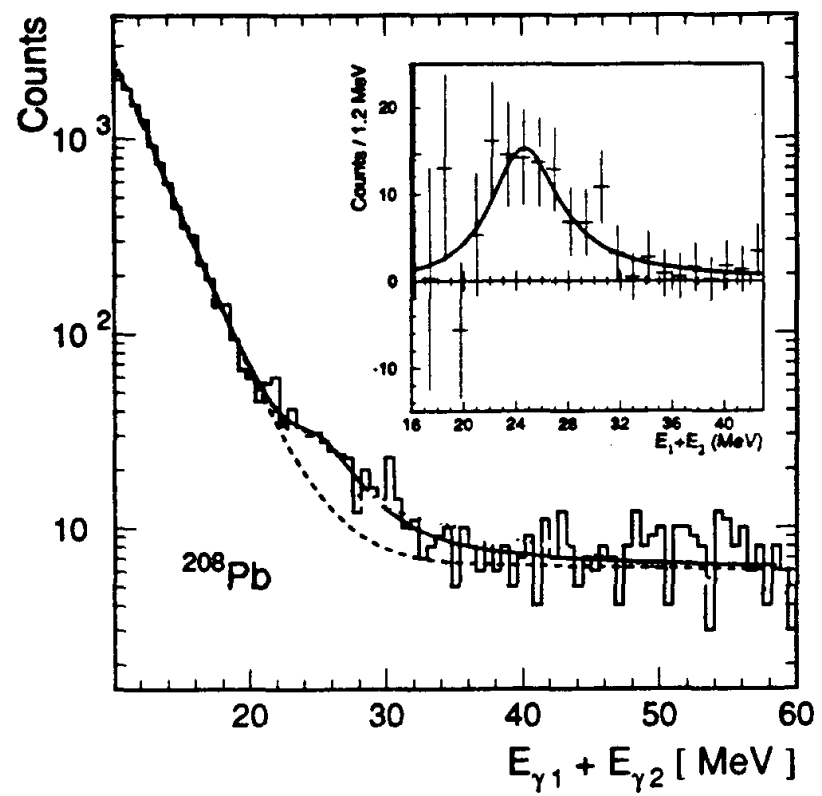

Novotny, 1991) comprising $256 \mathrm{BaF}_{2}$ scintillation counters which were grouped into four blocks. The experiment was semi-exclusive in a sense, that reactions accompanied by charged particle emission were strongly suppressed by means of a large array of plastic scintillators subtending a cone of $\pm 30^{\circ}$ of the laboratory forward hemisphere. In this way, peripheral interactions of the two heavy ions were selected. The Doppler shift affecting $\gamma$ rays emitted from a moving source was utilized in a two-fold way: The $\gamma$ detectors were placed at relatively large angles $\left(73^{\circ}-123^{\circ}\right)$ with regard to the beam axis and thus the effective solid angle for detection of $\gamma$ rays emitted from projectile-like fragments is strongly reduced. Moreover, because of the finite solid angle of the detector blocks, $\gamma$ rays from projectile fragments are Doppler smeared in their energy and thus appear as smooth background in the $\gamma$-energy spectra. $\gamma$ Rays emitted from target-like fragments appear unshifted in energy, since the momentum transfer in case of electromagnetic interactions (see chapter 3.1 ) is negligible. Two-fold $\gamma$-ray coincidences were analyzed under the constraint that the difference in photon energy of the two $\gamma$ rays is less than $6 \mathrm{MeV}$. This condition was chosen in order to enhance contributions from the twofold direct $\gamma$ decay of the DGDR. It was shown (Ritman, 1993b) that this condition is a bias of minor importance with respect to the deduced results. The distribution in sum energy of the coincident $\gamma$-ray pair as presented in (Ritman, 1993a) is shown in Fig. 18. The distribution could be described in terms of three components, i.e. a lorentzian distribution assigned io the DGDR double $\gamma$ decay in ${ }^{208} \mathrm{~Pb}$, a component exponentially rising towards lower energies ascribed to statistical $\gamma$ decays, and another exponential tail at higher energies. The latter one was ascribed to an incomplete suppression of more complex reactions or to one-step excitations into the quasideuteron region. A similar analysis was performed for the energy spectra obtained for the detection of single $\gamma$ 
rays. Applying Doppler-shift corrections adequate for $\gamma$ rays emitted from target-like fragments and alternatively for $\gamma$ rays from projectile fragments, spectra were obtained which resemble the direct groundstate $\gamma$ decay from the single giant dipole resonance in the ${ }^{208} \mathrm{~Pb}$ target and ${ }^{209} \mathrm{Bi}$ projectile. The deduced resonance parameters are in good agreement to those from photoabsorption measurements. The results for the DGDR are discussed in chapter 4.

An additional feature concerning the dynamics of the electromagnetic excitation process could be checked in this $\gamma$-decay study. Since the electromagnetic field is preferantially of transverse nature, the population of the $m= \pm 1$ magnetic substates of a giant dipole resonance with spin $\mathrm{I}^{\pi}=1^{-}$should be much stronger than that of the $\mathrm{m}=0$ substate. This leads to a characteristic $\gamma$-ray angular distribution. The angular distribution of $\gamma$ rays from GDR-to-groundstate transitions in ${ }^{208} \mathrm{~Pb}$ was found in good agreement to the one expected for electromagnetic excitation.

Another technique in studying the DGDR in ${ }^{208} \mathrm{~Pb}$, exploiting the direct $\gamma$-decay branch, was applied in a measurement performed at GANIL, Caen, by the Oak Ridge and GANIL groups. Inelastic scattering of ${ }^{17} \mathrm{O}(84 \mathrm{~A} \cdot \mathrm{MeV}),{ }^{36} \mathrm{Ar}(95 \mathrm{~A} \cdot \mathrm{MeV})$, and ${ }^{86} \mathrm{Kr}(60 \mathrm{~A} \cdot \mathrm{MeV})$, projectiles on ${ }^{208} \mathrm{~Pb}$ targets and of ${ }^{64} \mathrm{Zn}(80 \mathrm{~A} \cdot \mathrm{MeV})$ projectiles on ${ }^{209} \mathrm{Bi}$ targets was measured. Only preliminary data are available (Beene, 1993). The excitation energy was determined in an conventional way with the magnetic spectrometer SPEG. Since relatively low bombarding energies and low- $Z$ projectiles are used, only small DGDR cross sections are expected. In order to enhance the yield of the DGDR with regard to competing processes, the spectrometer was operated in coincidence to the photon spectrometer TAPS, selecting $\gamma$ rays from the decay of one of the DGDR phonons. Projectile scattering angles around the grazing angle were accepted by the magnetic spectrometer. Differential cross sections $d \sigma / d \Omega d E^{\star}$ as function of excitation energy $E^{\star}$, obtained by requiring a coincident $\gamma$ ray of energy $>9 \mathrm{MeV}$, are shown in Fig. 19. Adiabaticity effects at these low bombarding energies severely distort the DGDR response (see chapter 3 ) and non-negligible corrections have to be applied in extracting resonance parameters such as width and energy. Applying such corrections, preliminary values of 27.4(6) MeV and 6.5(1) MeV were deduced for the DGDR resonance energy and width in ${ }^{208} \mathrm{~Pb}$, respectively. The experimental spectra were compared to calculations with the folding model (Llope and Braun-Munzinger, 1990; see chapter 3.2), which are also shown in Fig. 19. In Fig. 20, the experimental cross sections integrated over excitation energies relevant for the DGDR are given in comparison to the calculated ones. It was assumed that the $\gamma$-branching ratio for DGDR and GDR are identical. Obviously the measured cross sections exceed the calculated ones by a factor 2 , an effect which will be discussed in the following chapter. 

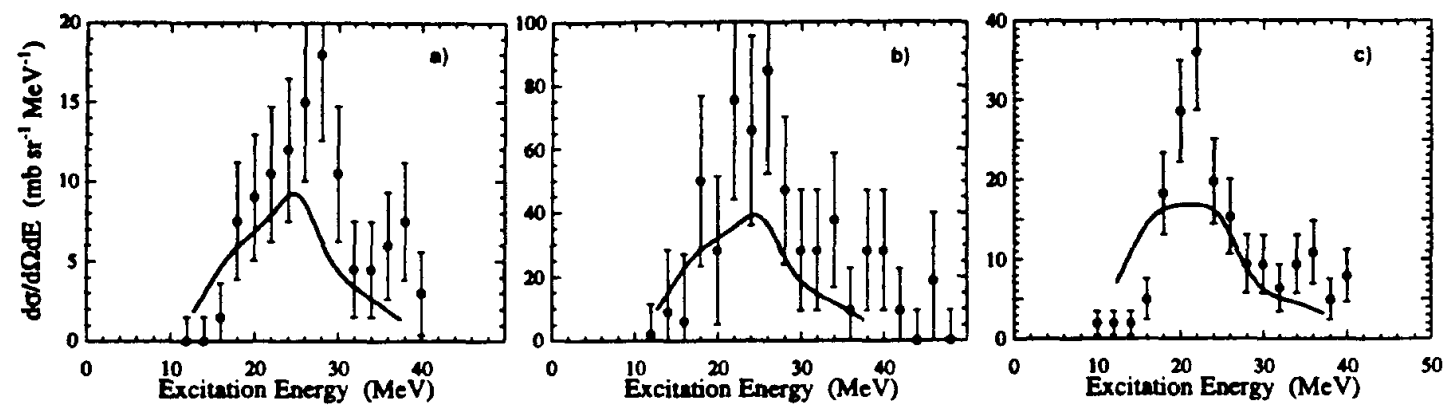

Fig. 19. Excitation spectrum for the two-phonon GDR state: a) ${ }^{36} \mathrm{Ar}(95 \mathrm{~A} \cdot \mathrm{MeV})$ on $\left.{ }^{208} \mathrm{~Pb}, \mathrm{~b}\right){ }^{64} \mathrm{Zn}$ $(80 \mathrm{~A} \cdot \mathrm{MeV})$ on ${ }^{208} \mathrm{Bi}$, and $\left.\mathrm{c}\right){ }^{86} \mathrm{Kr}(60 \mathrm{~A} \cdot \mathrm{MeV})$ on ${ }^{208} \mathrm{~Pb}$. Note the different cross section scales. The solid curves are essentially parameter-free calculations folding the photoabsorption cross section for the GDR with itself and the virtual photon spectrum to obtain the two-phonon excitation (Beene, 1993).
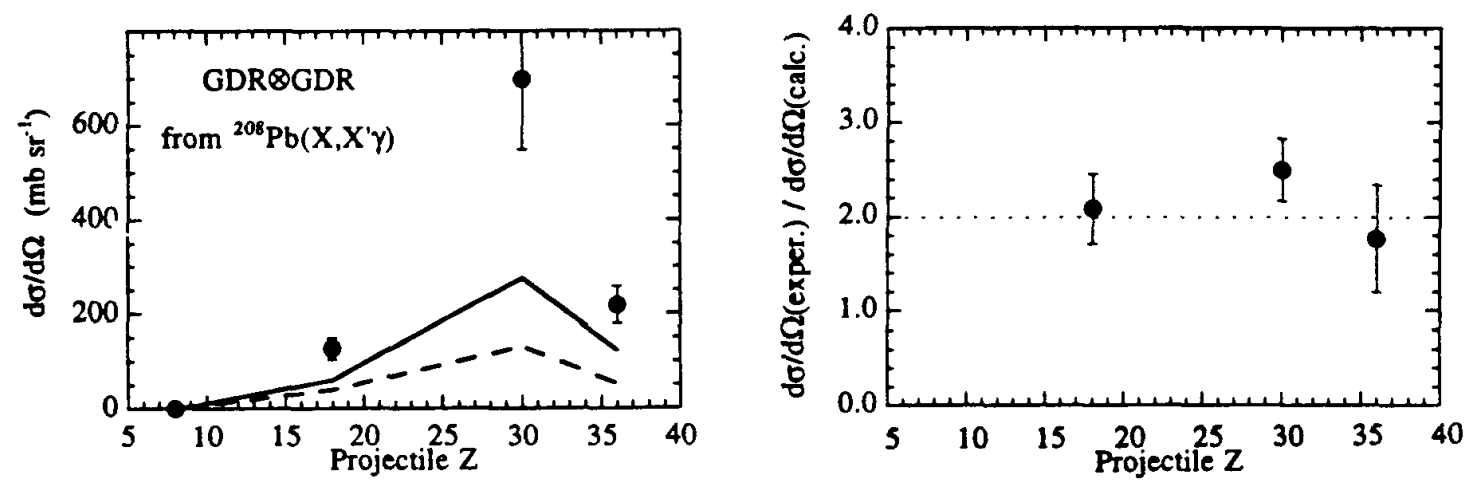

Fig. 20. Cross sections for DGDR excitation in ${ }^{208} \mathrm{~Pb}$. The left hand panel shows the energyintegrated cross sections from the data in Fig. 19 as a function of projectile charge $Z$. The solid line corresponds to the theoretical curves in Figure 19. The right hand panel is the ratio of experimental data to theory. The choice of $Z$ for an abscissa is for convenience. Since different projectile velocities are involved, the variation in cross section with $Z$ is not simply a $Z$ effect.

\subsection{Pion Double Charge Exchange Reactions}

First evidence for a two-phonon giant dipole resonance was reported from $\left(\pi^{+}, \pi^{-}\right)$double charge exchange reactions on a nals target (Mordechai et al., 1988). Fror the microscopic structure of the DGDR which is of two particle - two hole type, it is conceivable that the process of exchanging two protons and two neutrons can give rise to excitations of the DGDR. Moreover, the pion is a spinless $T=1$ particle and, therefore, no spin-flip, isospin-flip transitions are likely to be induced. Similar observations were made meanwhile on a number of targets ranging from ${ }^{12} \mathrm{C}$ to ${ }^{197} \mathrm{Au}$ (Mordechai et al., 1990; Grof et al., 1993). The data were taken at the Los Alamos Meson Physics Facility (LAMPF) using the EPICS set-up at pion energies of $295 \mathrm{MeV}$. For a ${ }^{56} \mathrm{Fe}$ target a measurement at a 


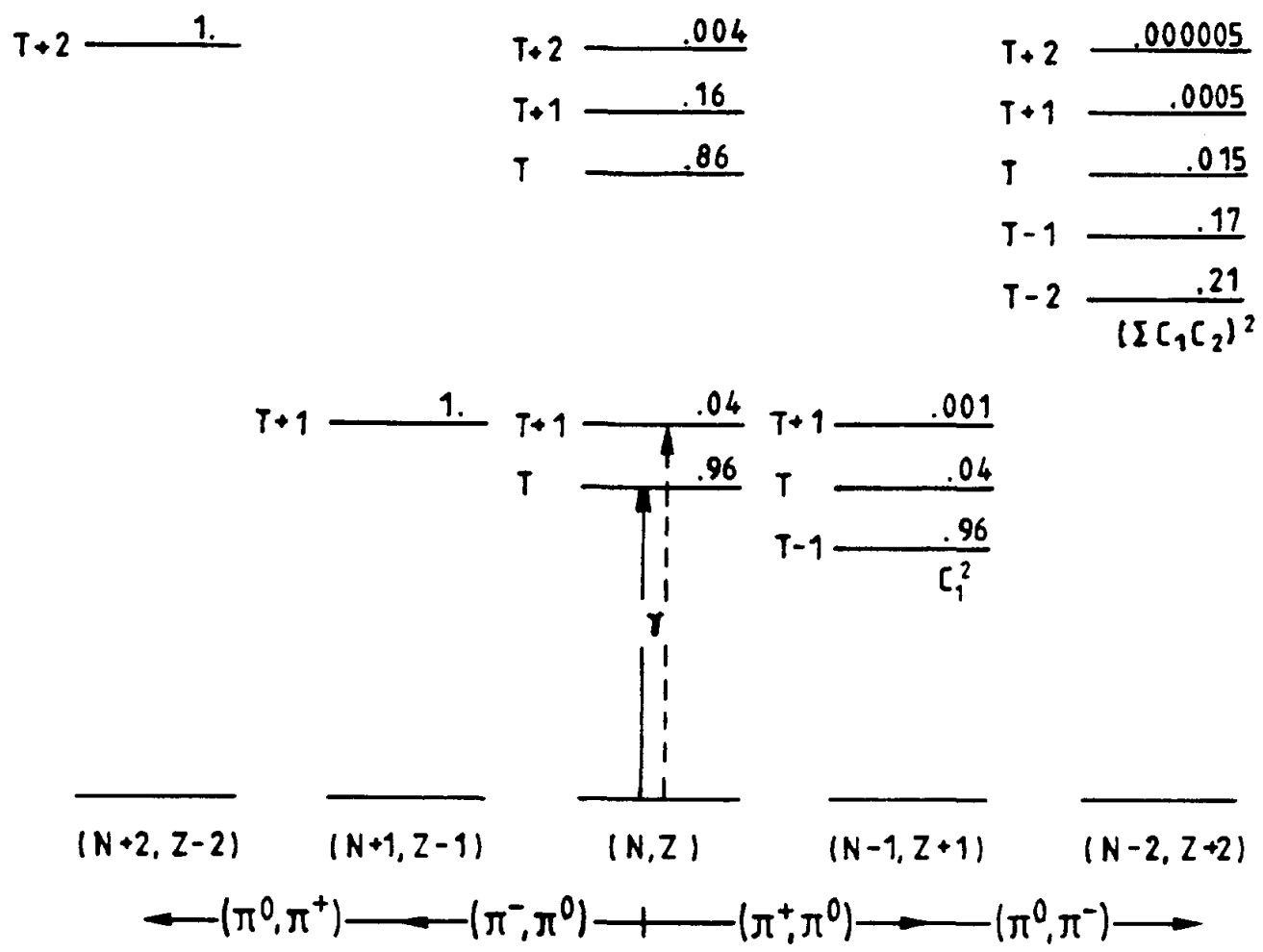

Fig. 21: Transition probabilities for inelastic excitations and charge exchange reactions on a nucleus with isospin $T=T_{2}=T_{0}$. For all transitions, a constant matrix element is assumed, the transition probabilities are then determined by the Clebsch-Gordan coefficients $C$, which are evaluated for the case of ${ }^{200} \mathrm{~Pb}$.

pion energy of $400 \mathrm{MeV}$ was performed, and for the ${ }^{40} \mathrm{Ca}$ target also the inverse reaction $\left(\pi^{-}, \pi^{+}\right)$was studied. Recent presentations in summary form are found in (Mordechai and Moore, 1991; 1993; Fortune, 1991).

A discussion of the two-phonon giant dipole resonance observed in heavy ion electromagnetic scattering cannot be complete without contrasting the results with those available from the pion measurements. One has to be aware, however, that a comparison is not straight forward, since double charge exchange reactions populate giant resonances in a neighbouring nucleus with the isospin component $T_{2}$ differing by 2 units from that of the target nucleus. This effect is illustrated in Fig. 21. Transition probabilities between the various members of isospin multiplets are governed by the isospin Clebsch-Gordan coefficients (Satchler, 1983) and the corresponding matrix elements. Transition probabilities shown in Fig. 21 are evaluated for ${ }^{208} \mathrm{~Pb}$, assuming a constant matrix element for the various transitions. Evidently, in heavy nuclei with large neutron excess and thus $T_{z} \gg 1$, preferentially the lowest member of the isospin multiplett should be excited, i.e. $\left(T, T_{2}\right)=\left(T_{0}-2, T_{0}-2\right)$ in a $\left(\pi^{+}, \pi^{-}\right)$reaction. For lighter nuclei, however, isospin splitting effects can strongly affect the width observed for the DGDR. In that case, a comparison with data from Coulomb excitation, where predominantly $T=T_{0}$ is excited, might be misleading. For this reason, the comparison is restricted to heavy nuclei of $A \gtrsim 100$. We include, however, the result for ${ }^{40} \mathrm{Ca}$, which is a self-conjugate nucleus $\left(T_{o}=0\right)$, and thus only transitions to $T=2$ states are possible. 
Fig. 22. Doubly differential cross section spectra for the ${ }^{197} \mathrm{Au}\left(\pi^{+}, \pi^{-}\right)$reaction, $Q$ denotes the reaction $Q$-value. The solid line reflects a fit to the data involving the DIAS and GDRxIAS (see text) and the double giant dipole resonance (GDR $\times$ GDR) and a parametrized background (dashed line).

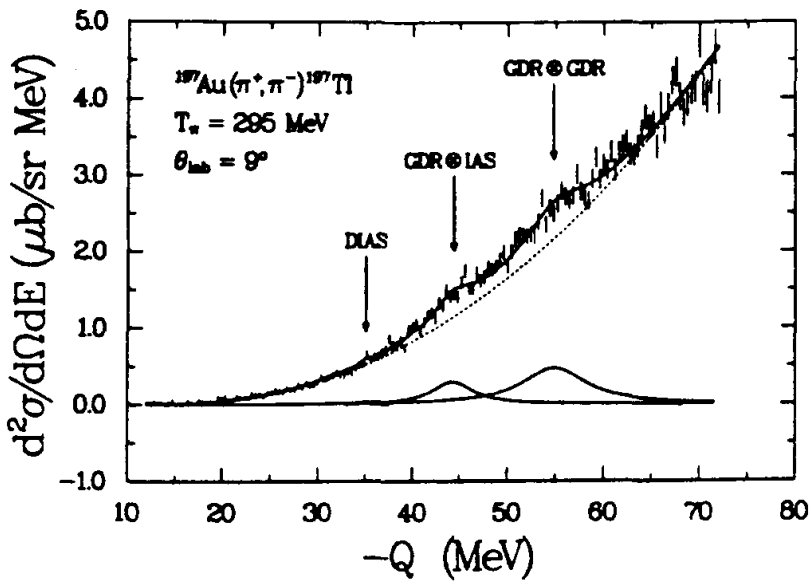

The DGDR data presently available from $\left(\pi^{+}, \pi^{-}\right)$reactions are summarized in Table 2. A spectrum representative for the quality of the data is shown in Fig. 22 for the heaviest nucleus ${ }^{197} \mathrm{Au}$. It is noticed that the giant resonances appear superimposed on a considerable background, which is not understood quantitatively and has to be parametrized for subtraction. Other structures showing up in $\left(\pi^{+}, \pi^{-}\right)$spectra are the double isobar analog state (DIAS) and the GDR built onto the isobar analog state (GDR $\times$ IAS), for a discussion of which we refer to (Mordechai and Moors, 1991; 1993: Clement, 1992).

Spectra obtained from $\left(\pi^{+}, \pi^{-}\right)$and the inverse $\left(\pi^{-}, \pi^{+}\right)$reaction on ${ }^{40} \mathrm{Ca}$ are shown in Fig. 23. Since ${ }^{40} \mathrm{Ca}$ has isospin $\mathrm{T}=0$ both the DIAS and IASXGDR states must be absent and the $T=2$ DGDR state are populated in both reactions. The two spectra in fact can be described with consistent assumptions on the DGDR width and on the background (solid curve in Fig. 23). The observed difference in reaction Q-value is consistent with the expected value resulting from the Coulomb displacement energy and proton-neutron mass difference (see below).

Fig. 23. Double differential cross section for the reaction ${ }^{40} \mathrm{Ca}\left(\pi^{-}, \pi^{+}\right)$(upper panel) and the ${ }^{40} \mathrm{Ca}\left(\pi^{+}, \pi^{-}\right)$ reaction (lower panel). Data are taken from (Mordechai and Moore, 1991). The position of the DGDR states and the ground states are indicated.

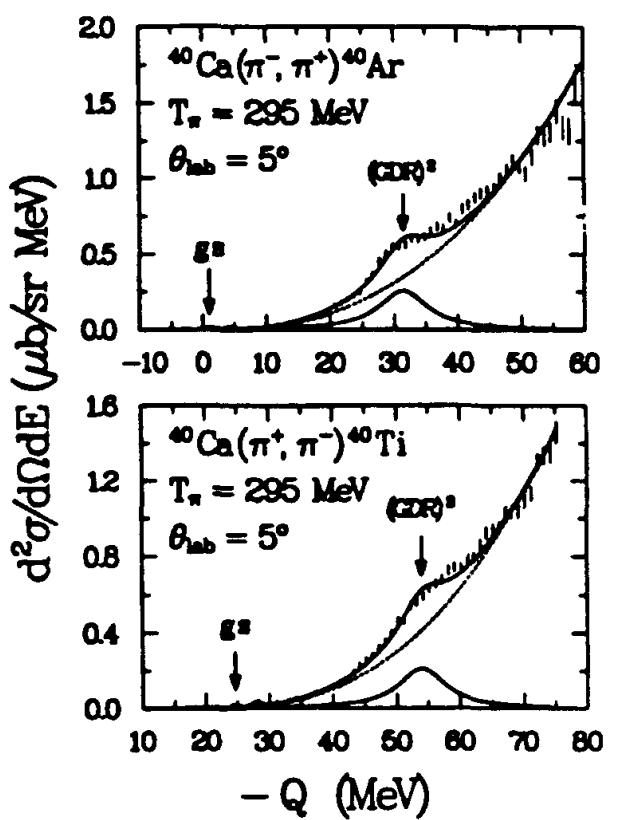


Table 2: Reaction Q-values, width $\Gamma$ and approximate total cross sections $\sigma_{t}$ as measured for the DGDR in $\left(\pi^{+}, \pi^{-}\right)$reactions. Resonance energies $E$ and ratios $R$ of experimental to calculated cross sections, measured at $5^{\circ}$, are given as well (see text for details).

\begin{tabular}{lccccc} 
Target Nucleus & $-\mathrm{Q}[\mathrm{MeV}]$ & $\Gamma[\mathrm{MeV}]$ & $\sigma_{\mathrm{t}}[\mu \mathrm{b}]^{\mathrm{a}}$ & $\mathrm{E}[\mathrm{MeV}]$ & $\mathrm{R}^{\mathrm{b}}$ \\
${ }^{40} \mathrm{Ca}^{\mathrm{C}}$ & $51.1(5)$ & $9.0(1.4)$ & 12.9 & $37.2(5)$ & $3.5(3)$ \\
${ }^{93} \mathrm{Nb}^{\mathrm{C}}$ & $49.9(0.8)$ & $8.8(2.6)$ & 17.8 & $31.8(8)$ & $3.9(5)$ \\
${ }^{115} \mathrm{n}^{\mathrm{d}}$ & $49.6(8)$ & $8.5(3.0)$ & 66.3 & $31.8(8)$ & - \\
${ }^{138} \mathrm{Ba}^{\mathrm{C}}$ & $49.8(8)$ & $8.5(2.6)$ & 23.6 & $30.4(8)$ & $5.1(6)$ \\
${ }^{197} \mathrm{Au}^{\mathrm{d}}$ & $54.9(8)$ & $9.0(3.0)$ & 41.2 & $25.2(8)$ & $4.7(1.6)$ \\
\hline
\end{tabular}

a (Bar-Touv and Mordechai, 1992)

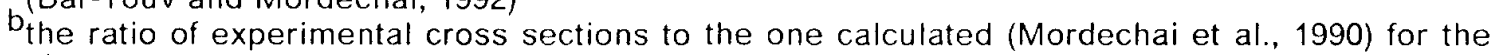
$2^{+}$member is given

C(Mordechai et al., 1990)

d(Grof et al., 1993)

In order to be able to compare DGDR data from pion reactions to those from heavy ion scattering, as attempted in the next chapter, one has to correct the observed resonance energy for the Coulomb displacement energy due to the successive transfer of two charges, for the proton-neutron mass difference, and for shifts caused by the isospin splitting. Such a procedure was proposed in (Bar-Touv and Mordechai, 1992) to which we refer for details. Therein, empirical relations (Anderson et al., 1965; Antony et al., $1985)$ are used. Adopting this method, corrected resonance energies are included in Table 2. Since only heavy nuclei ar considered for reasons discussed above, isospin splitting should not affect very much the wicth of the DGDR state. It is, however, an open question, to which extent the reaction dynamics modifies the strength distribution. The width, quoted in Table 2, was extracted adopting lorentzian shapes in analyzing the experimental spectra. Estimated total cross sections for the DGDR are also quoted in Table 2. Obviously, these cross sections are about 5 orders of magnitude smaller than those obtained with heavy ions.

Several questions remain open which concern, in particular, the observed transition strength. Usually, measured differential cross sections $\mathrm{d} \sigma / \mathrm{d} \Omega$ are compared to calculations on the basis of a coupled-channel impulse-approximation (CCIA) and using collective Tassie form factors for the transition density (Tassie, 1978). The differential cross sections essentially are consistent with an angular momentum transfer $\Delta \mathrm{L}=2$ with rather small admixtures of $\Delta L=0$ (Mordechai et al., 1988; 1990; Grof. et al., 1993). The DGDR couples to spins $\mathrm{I}^{\pi}=0^{+}, 2^{+}$, however, and the calculation (Mordechai et al., 1990; performed for an observation angle of $5^{\circ}$ ) predict a $\Delta L=0$ component stronger than the $\Delta \mathrm{L}=2$ component. The mechanism which suppresses the $\Delta \mathrm{L}=0$ component, is unknown. The ratio of measured to calculated cross sections at an angle of $5^{0}$, taking into account the $\Delta L=2$ component, which represents most of the experimental cross, is included in Table 2. Under such assumptions, the experimental values exceed those of the calculation by a factor of about 4 to 5 . A similar factor of 5.9 is quoted (Smith et al., 1993) for 
Fig. 24. Differential cross sections at $5^{\circ}$ scattering angle, normalized to $(N Z)^{2}$ (see text) for the double isovector giant dipole resonance (GDR $\times$ GDR) excited in $\left(\pi^{+}, \pi^{-}\right)$reactions at $295 \mathrm{MeV}$ (Grof et al., 1993).

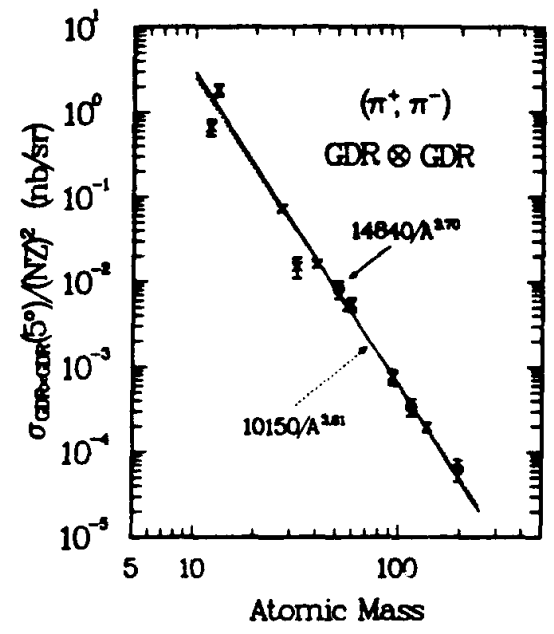

a measurement on ${ }^{56} \mathrm{Fe}$ at a higher pion energy of $400 \mathrm{MeV}$. Therein, it is also observed that the dependence of the DGDR cross section on bombarding energy is not well reproduced, although the CCIA calculation predicts reasonably well the energy dependence of the DIAS and DIAS $x$ GDR two-phonon states. These observations are summarized (Fortune, 1991), stating that for the $0^{+}$and the $2^{+}$DGDR members in pion double charge exchange reactions one obstrves

$$
\sigma_{\exp }\left(0^{+}\right) \lesssim \sigma_{c a l c}\left(0^{+}\right) / 3 ; \sigma_{\exp }\left(2^{+}\right)=(2-3) \sigma_{c a l c}\left(2^{+}\right) .
$$

The lack of $L=0$ strength can hardly be checked in heavy ion induced reactions since the excitation of the $2^{+}$member is predominant (see chapter 5.4 ).

Nevertheless, a global dependence of the differential cross section on target mass and charge was observed (Mordechai et al., 1930; Grof, et al., 1993), which can be written as

$$
d \sigma / d \Omega=N^{2} Z^{2} / A^{\alpha}
$$

and which is very well obeyed, as shown in Fig. 24. This global dependence can be qualitatively understood if the cross section is factorized into strength, governed by the E1 sum rule (see chapter 2.3), times a mass dependent attenuation factor due to distortions of the pion waves inside the nucleus. With the present poor knowledge on the pion propagation in the nuclear medium, however, one might still debate if the transition strength can be extracted reliably from measured pion double charge exchange cross sections.

\subsection{Two-Phonon Isoscalar Giant Quadrupole Resonance}

Aside from the DGDR, evidence for two-phonon states has been reported so far only for the isoscalar quadrupole resonance. Using medium heavy ions at moderate bombarding energies of several tens of A.MeV, nuclear excitations are stronger or at least comparable to electromagnetic giant resonance excitations. Nuclear isoscalar excitations in general interfere constructively with the electromagnetic excitation while the interference 
Fig. 25. Excitation energy spectrum obtained in the reaction ${ }^{10} \mathrm{Ca}\left({ }^{40} \mathrm{Ca}\right.$, ${ }^{40} \mathrm{Ca}$ ) at $44 \mathrm{~A} \cdot \mathrm{MeV}$ in coincidence to protons emitted at backward angles. The structure arising around $34 \mathrm{MeV}$ excitation energy is assigned to the double isoscalar giant quadrupole resonance (Scarpaci et al., 1993; Frascaria, 1993).

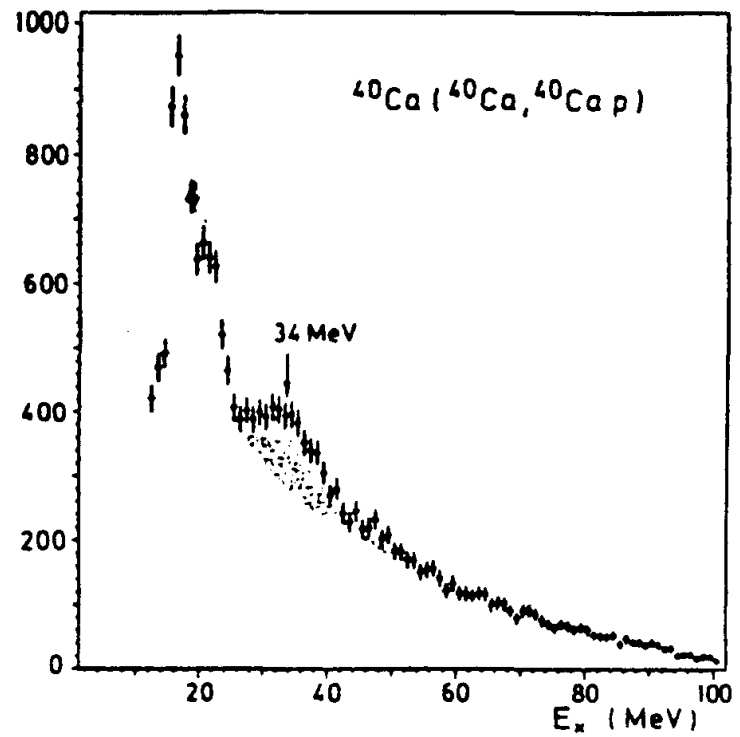

the interference is destructive for isovector excitations $\left(T_{z} \geq 1\right)$ (Satchler, 1983). Since isovector giant quadrupole resonances are higher in excitation energy, their excitation is suppressed in comparison to the isocalar quadrupole resonance. Medium-mass ions at bombarding energies of several tens of A.MeV thus suit well for studying multi-phonon states of isoscalar quadrupoie resonances. In measurements using $\mathrm{Ar}$ and $\mathrm{Ne}$ projectiles on ${ }^{208} \mathrm{~Pb}$ targets (Chomaz et al., 1984a) weak structures on top of a large background were observed which were claimed to be consistent with predictions for multi-phonon GQR excitations (Chomaz et al., 1984b). These structures were not reproduced in subsequent measurements (Bertrand et al., 1987) giving rise to some contraversies. For an extended discussion we refer to (Frascaria, 1988 and refs. therein).

Here we concentrate on a recent experiment which gave a clean experimental signature, obtained in a semi-exclusive measurement for the $\mathrm{Ca}+\mathrm{Ca}$ system at $44 \mathrm{~A} \cdot \mathrm{MeV}$ bombarding energy performed at GANIL. Inelastically scattered Ca projectiles, analyzed with a magnetic spectrometer, are registered in coincidence to protons emitted at backward angles (Scarpaci et al., 1993; Frascaria, 1993). The resulting spectrum as shown in Fig. 25 exhibits a prominent structure, centered around $34 \mathrm{MeV}$ excitation energy. In the inclusive spectrum not shown here, a peak at $17.5 \mathrm{MeV}$ and a weaker component at 14 . $\mathrm{MeV}$ are observed, both being ascribed to an excitation of the single isoscalar GQR. As appearing at about twice the GQR excitation energy, the peak at $34 \mathrm{MeV}$ in the proton coincident spectrum is assigned to the two-phonon state. The assignment is supported by a detailed study of the proton decay characteristics. The parameters quoted for the double isoscalar GQR are a mean energy of $34 \mathrm{MeV}$ and a width of $9 \pm 2 \mathrm{MeV}$. The cross section of the double relative to the single resonance is given to be $\sim 0.125$ while the reaction model of (Blumenfeld and Chomaz, 1988) predicts a value near 0.07 , thus underestimating the relative cross section by a factor of about two. An accurate determination of cross sections, however, is hampered by the fact, that the threshold for emission of two protons is near to the energy of the double GQR. 
Table 3: Parameters of the DGDR obtained from heavy ion induced electromagnetic excitation and from $\left(\pi^{+}, \pi^{-}\right)$reactions. $E(D G D R), \Gamma(D G D R)$ and $\sigma(D G D R)$ denote the energy of maximum cross section, the width (FWHM) and total cross section, respectively.

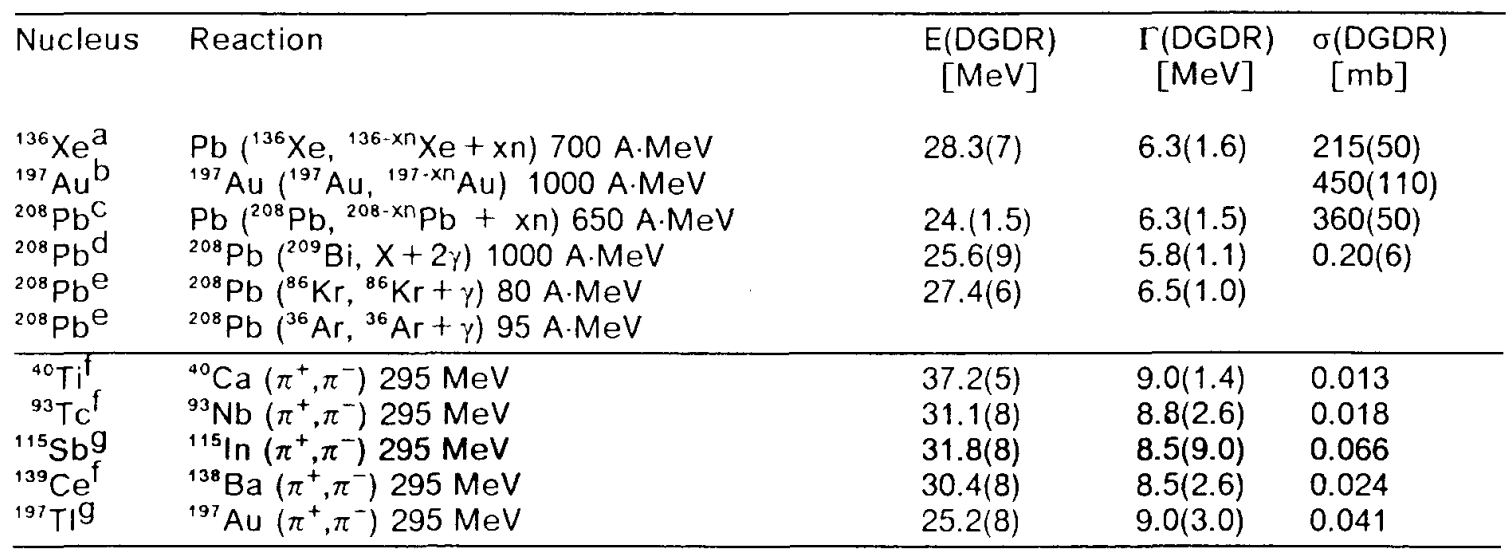

a (Schmidt et al., 1993)

b (Aumann et al., 1993); the cross section was extracted as discussed in chapter 4.1

$\mathrm{C}_{\text {(Wajda et al., 1993); preliminary values }}$

d(Ritmann et al., 1993); the cross section is that for the double $y$ decay

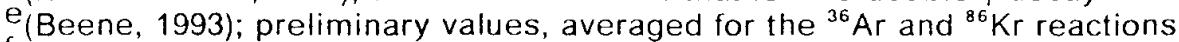

(Mordechai et al., 1990)

(Smith et al., 1993)

\section{DISCUSSION}

\subsection{Summary of the DGDR Data}

After having discussed the various experiments which yielded evidence for the heavy ion induced electromagnetic excitation of the two-phonon isovector giant dipole resonance, the experimental results are summarized. These data are combined with the information deduced from pion double charge exchange experiments. In the following three subsections, first the parameters describing the strength distributions are discussed, then the results from neutron and $\gamma$ decay, and finally the observed cross sections, whose understanding is still a puzzle. Simultaneously, theoretical approaches relevant for a description of the DGDR and contrasting the experimental data are presented.

A survey of the experimental results which are presently available from heavy ion scattering and $\left(\pi^{+}, \pi^{-}\right)$reactions is given in Table 3 . The resonance energy (i.e. energy of maximum cross section), width (FWHM) and total cross sections are quoted. It should be noted, that these numbers, in most cases were extracted adopting a lorentzian or gaussian distribution, since the data in general are not sensitive to weak components or extended wings. Resonance energies from $\left(\pi^{+}, \pi^{-}\right)$reactions were extracted as discussed in chapter 4.4. For reasons also discussed there, we consider $\left(\pi^{+}, \pi^{-}\right)$data only for heavy nuclei and ${ }^{40} \mathrm{Ca}$. 


\subsection{Strength distribution}

In Table 3 the lowest moments of the DGDR strength distribution are listed as observed experimentally. Within the statistical accuracy, the measured distributions are compatible with a single lorentzian distribution, but in most cases would also be consistent with a gaussian distribution. We recall that the simple model of independent harmonic oscillator quanta (see chapter 2.4) implies that the energy of the two quanta is identical and, because both quanta are excited and decay independently, the width of a twophonon state should be twice that of the one-phonon state. Thus one expects

$$
\frac{E(D G D R)-E(G D R)}{E(G D R)}=1 \text { and } \frac{\Gamma(D G D R)}{\Gamma(G D R)}=2
$$

The validity of these simple relations are checked in Fig. 26 . It is observed that both values, that for the energy as well as for the width, are smaller than those expected for harmonic vibrations. The values, averaged over all nuclei, for the relative energy of the second phonon and the relative width of the DGDR as shown in Fig. 26 amount to $0.91 \pm 0.02$ and $1.60 \pm 0.11$, respectively. If these numbers were evaluated separately for the heavy ion data and the pion reactions one derives for the relative energy a mean value of $0.92 \pm 0.03$ and $0.91 \pm 0.02$, and for the relative width $1.50 \pm 0.15$ and $1.67 \pm 0.20$, respectively. There is thus no significant difference between the results obtained from two different experimental methods. Considering the relative energy of the second phonon one is led to the conclusion that anharmonicities are of the order of $10 \%$, reducing the amplitude of the collective vibration by about $5 \%$.

Before discussing the DGDR width, we emphasize again that the following effects in principle could broaden the DGDR strength distribution:

a) due to anharmonicities the DGDR spin multiplet $\mathrm{I}^{\pi}=0^{+}, 2^{+}$could be non-degenerate,

b) isospin-splitting effects as discussed earlier,

c) static deformations could lead to strength fragmentation as known for the GDR.

The selection of data (see chapter 4.4) was aiming on discarding nuclei which could be influenced from isospin effects, and furthermore, the nuclei considered here are (semi) magic or are known to have small static deformations. As deduced from the DGDR energy, anharmonicities obviously are very small and therefore, the $0^{+}$and $2^{+}$spin states should not much differ in energy. Consequently, it is supposed that energies and width as shown in Fig. 26 reflect those of a strength distribution, which is basically not affected by fragmentation due to the effects mentioned above.

It is common believe that the apparent width of the giant dipole is determined by its mixing with non-resonant states of more complex structure (spreading width). It is conceivable that the width of the double giant dipote resonance is as well dominated by coupling to non-resonant continuum states. More precisely, strongest coupling is expected to states consisting of one collective phonon plus a non-collective phonon, the latter one representing the same background states which are responsible for the GDR spreading width. Some fundamental arguments were used (Bertulani and Zelevinsky, 1993) to estimate the spreading width of two- and higher-phonon states. They note that a distribution equivalent to a lorentzian one is obtained assuming an equidistant level 

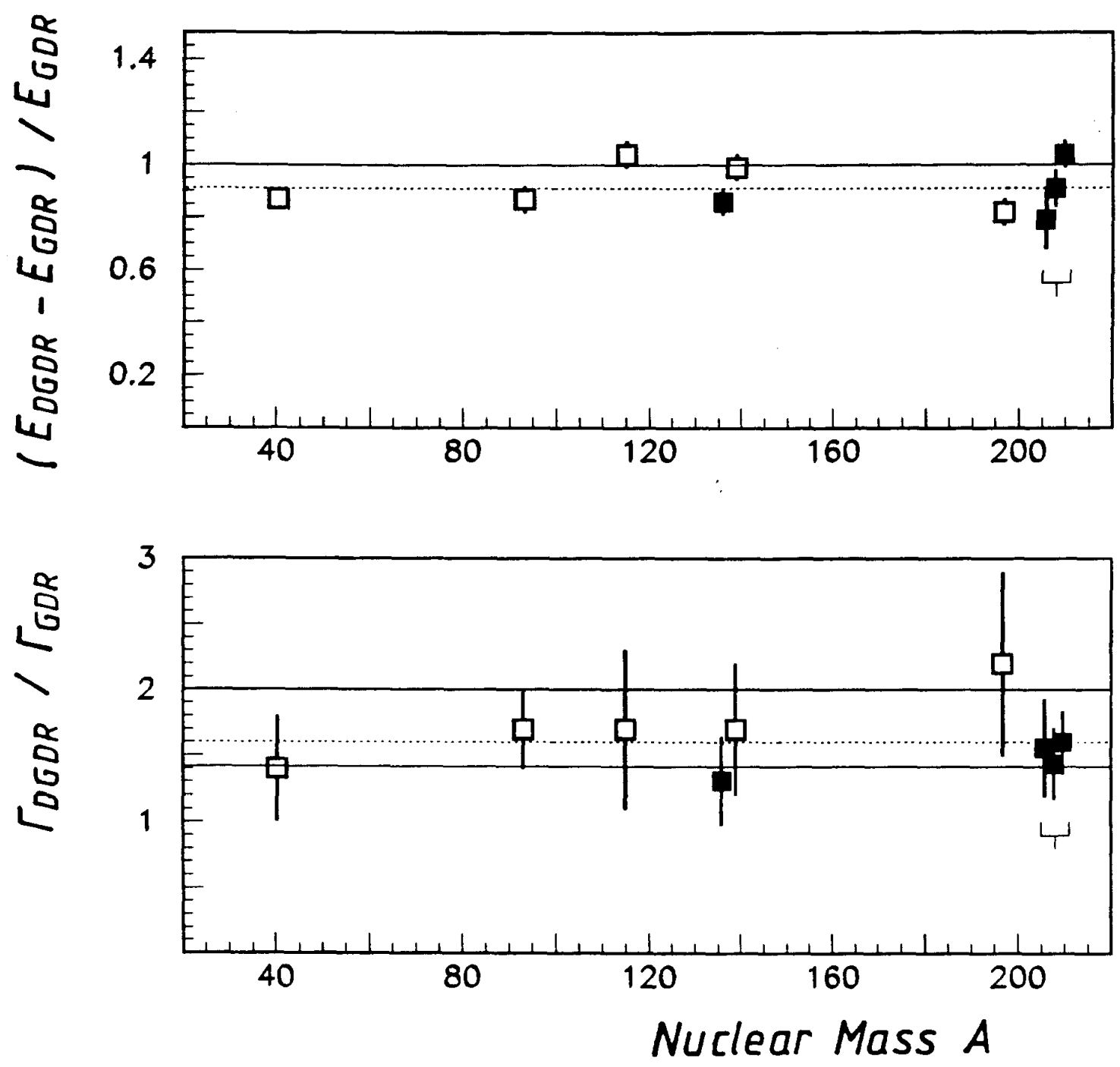

Fig. 26. (upper panel) Energy of the second phonon of the DGDR, relative to the energy of the single-phonon GDR. Open symbols denote data from $\left(\pi^{+}, \pi^{-}\right)$reactions, full symbols denote data from heavy ion induced electromagnetic excitation. The dashed line represents the mean value of all data, the solid line indicates the harmonic value.

(lower panel) Same as above, however, for the DGDR width relative to the GDR width. The solid lines indicate the theoretical limits $\Gamma(D G D R) / \Gamma(G D R)=2$ and $\Gamma(D G D R) / \Gamma(G D R)=\sqrt{2}$.

spacing $d$ between the background states and a constant mean matrix element $V$ coupling these states to the collective mode (Bohr and Mottelson, 1975); the spreading width is then

$$
\Gamma=2 \pi \frac{<v^{2}>}{d}
$$


Each of the collective phonons can be 'destroyed' by coupling to the background states, which are the same as for the one-phonon state and of same level spacing $d$, but the coupling matrix element $V$ has to be multiplied by the Bose factor $\sqrt{n}$, and in consequence one obtains again $\Gamma_{n}=n \Gamma_{1}$. In the same reference, arguments were also provided for a dependence of the form $\Gamma_{n}=\sqrt{n} \Gamma_{1}$. Considering a distribution which contrary to a lorentzian one - has a finite second moment, for example a gaussian distribution, one obtains the $\sqrt{n}$ dependence by convoluting $\mathrm{n}$ uncorrelated excitation steps. The basic difference to the lorentzian distribution is that the far remote state have a weaker coupling. In this context, one should also notice that the Coulomb excitation process truncates the high energy wings of the strength distribution due to the adiabatic cut-off. In addition, a lorentzian distribution fails to reproduce photoabsorption data on the low energy side and must be multiplied with a gaussian cut-off in order to fit the data. Such cut-off factors result in finite moments for the strength distribution and allows the use of the variance as a measure of the width. Such arguments were also presented in (Chomaz and Van Giai, 1992) and the authors conclude that for the width of a two-phonon state one might expect

$$
\Gamma(D G D R)=\sqrt{2} \Gamma(G D R)+\Delta .
$$

The correction $\triangle$ arises from small anharmonicities of the collective states, which on the basis of RPA calculations is expected to be of the order of $1 \mathrm{MeV}$ as discussed in chapter 2.4. The experimentally observed averaged value $\Gamma(D G D R) / \Gamma(G D R)=1.60 \pm 0.11$ is consistent with above relation.

The standard theoretical method for a description of single giant resonances in a miscroscopic basis is the random phase approximation (RPA) where residual interactions are diagonalized in the space of $1 \mathrm{p} 1 \mathrm{~h}$ excitations. For double giant resonances an extension of the RPA is required, where the Hamiltonian has to be diagonalized in the space of $2 \mathrm{p} 2 \mathrm{~h}$ states in addition to the $1 \mathrm{p} 1 \mathrm{~h}$ configurations (second RPA). Because of the complexity of the basis, however, various approximations and truncation schemes have to be applied. Calculations within the quasi-particle phonon model were performed for ${ }^{136} \mathrm{Xe}$ (Ponomarev et al., 1993). The phonon basis states are obtained solving the RPA equations with an effective Hamiltonian (Soloviev et al., 1977) which includes a WoodSaxon mean field, monopole pairing interaction and residual interaction in separable form. The diagonalization in the one- and two-phonon space leads to the mixed states, whose strength distributions fit rather well the empirical parameters of giant dipole and quadrupole resonances. The calculated strength distribution which naturally is of discrete type, was somewhat smoothed using an energy averaging parameter of $0.5 \mathrm{MeV}$. These distributions served as input into a semiclassical first- and second-order perturbation calculation for the electromagnetic excitation process in the system ${ }^{136} \mathrm{Xe}(700$ $\mathrm{A} \cdot \mathrm{MeV})+\mathrm{Pb}$ as measured by (Schmidt et al., 1993). The cross section for the double excitation of the isovector giant dipole resonance was determined in second-order perturbation theory. The result of the calculation is shown in Fig. 27. 


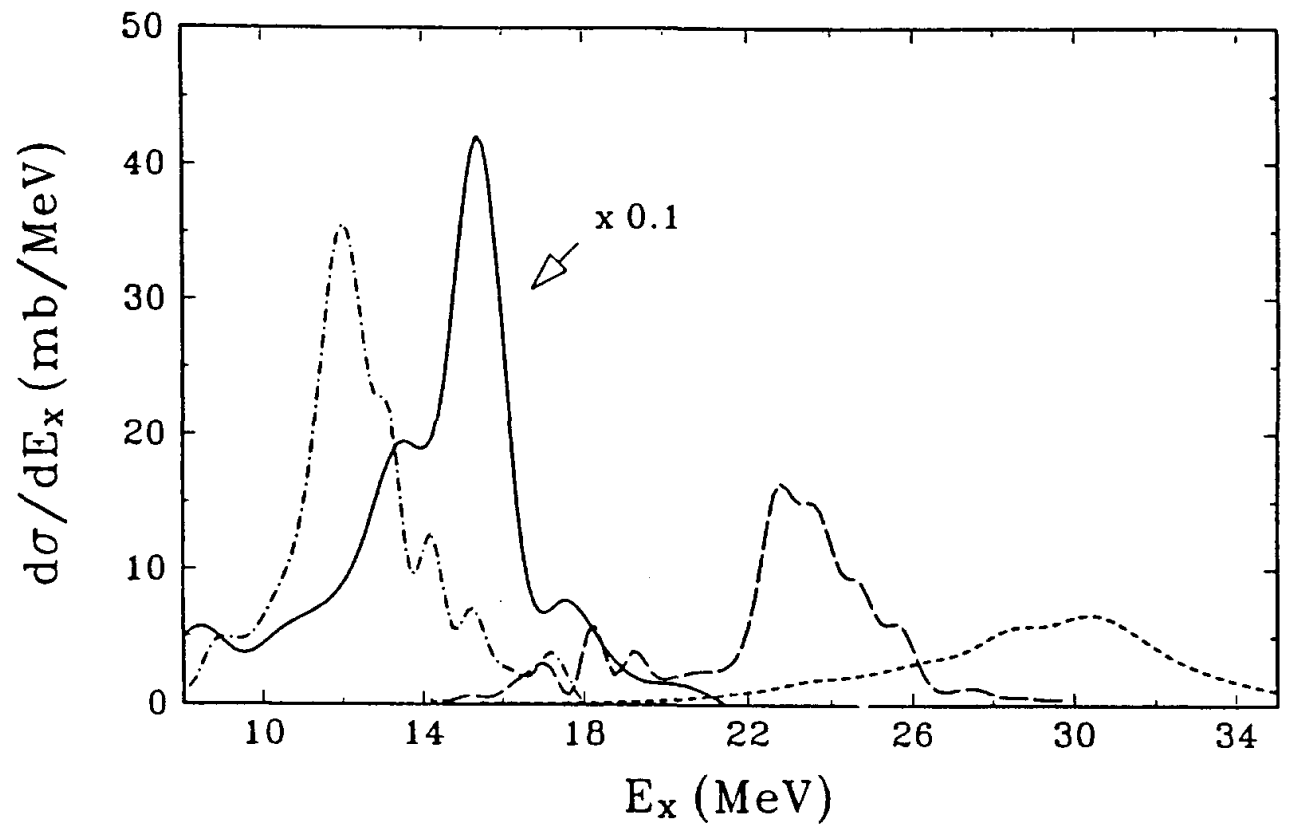

Fig. 27. Differential cross section with respect to excitation energy for Coulomb excitation of ${ }^{136} \mathrm{Xe}$ (see text), including the giant dipole resonance (solid line), and the isoscalar (dashed dotted) and isovector (long dashed) giant quadrupole resonance, calculated in first-order perturbation theory. The double excitation of the giant dipole resonance (short dashed) is obtained from second-order perturbation theory. The strength distribution of the one-phonon states is calculated within the quasi-particle phonon model (Ponomarev et al., 1993).

The distribution in cross section for the DGDR is of particular interest. It aquires a maximum cross section around $\sim 30 \mathrm{MeV}$ and the apparent width (FWHM) amounts to $6 \mathrm{MeV}$, being well in agreement with the experimental values quoted in Table 3 . However, again the assumption of an independent excitation of the two phonons is underlying, and thus the result of the second-order perturbation calculation is very similar to what one would obtain from the folding model (chapter 3.2). The quasi-phonon model was applied (Ponomarev and Voronov, 1992) also to the case of ${ }^{208} \mathrm{~Pb}$, however, using a more truncated basis. A Coulomb excitation calculation is not performed. Instead, the $B(E 2)$ strength distribution for two GDR phonons coupled to a $2^{+}$state was calculated, which not necessarily is representative of the cross section distribution measured in a two-step Coulomb excitation, since it applies to a single-step E2 excitation. The B(E2) distribution is shown in Fig. 28. A value of $25.5 \mathrm{MeV}$ for the centroid energy and of 4.4 MeV for the width (FWHM) of the DGDR in ${ }^{208} \mathrm{~Pb}$ is obtained (see also chapter 2.4), still compatible witl the experimental results of (Wajda et al., 1993) and (Ritmann et al., 1993). As seen from Fig. 28, the $B(E 2)$ values are rather small, and thus an electromagnetic single-step E2 excitation process will yield cross sections, much smaller than obtained for the two-step E1 excitation. The quasi-particle phonon approach applied by Ponomarev and collaborators does not include couplings of the two-phonon GDR to $3 \mathrm{p} 3 \mathrm{~h}$ and more complex configurations. Since the calculated width, however, is in reasonable agreement with the experimentally observed one, one might conclude, that such couplings do not widen considerably the DGDR strength distribution. 
Fig. 28. E2 strength distribution for transitions from groundstate into the DGDR $\left(1^{*}=2^{+}\right)$in ${ }^{208} \mathrm{~Pb}$ (Ponomarev and Voronov, 1992).

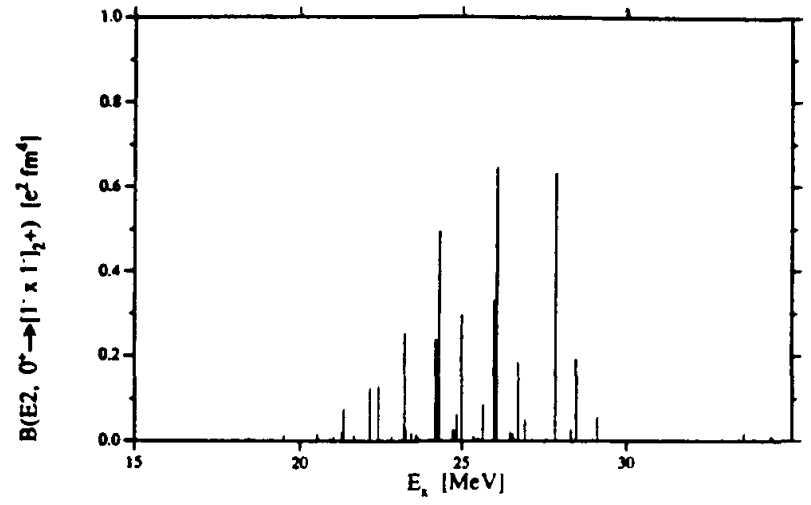

A second RPA calculation for ${ }^{208} \mathrm{~Pb}$ and light nuclei was carried out (Lauritsch and Reinhard, 1990) using a Skyrme-Hartree-Foc: model (Reinhard and Friedrich, 1985) and separable residual interactions. They calculate the strength distribution, connecting the two-phonon giant dipole state with its one-phonon states, and find it centered around $24 \mathrm{MeV}$ in the case ${ }^{208} \mathrm{~Pb}$, in good agreement with experimental data. For the fragmentation width of the DGDR in ${ }^{208} \mathrm{~Pb}$ they observe a relation $\Gamma(D G D R) \approx 1.4-1.6 \Gamma(G D R)$. They also make an empirical estimate of the spreading and escape width, however, overestimating the measured width. The temperature-dependent random-phase approximation (TDRPA) was employed for a calculation of the DGDR parameters (Bar-Touv and Mordechai, 1992). In the TDRPA, the DGDR is treated as a giant dipole resonance built on top of an excited state which is of same energy as the GDR, but of non-collective nature. Such an approach appears to us inappropriate since it misses the basic feature of a two-phonon giant resonance, i.e. the fact that all of the available energy is consumed into collective motion rather than in single particle degrees of freedom. Double giant dipole excitations were also treated in the SU(3) limit of the interacting boson model (Scholtz and Hahne, 1990), which is not discussed here since it applies to strongly deformed nuclei.

Summarizing the theoretical efforts, one realizes that the present miscroscopic methods, basically by construction, resemble a close analogy to the case of two independent harmonic oscillator quanta. Surprisingly, the calculated fragmentation width seems to exhaust the experimentally observed width to a large extent, indicating that mixing with more complex configurations $(3 \mathrm{p} 3 \mathrm{~h} .$.$) is relatively weak, and by comparing the RPA$ calculations with the experimentally observed width, one might estimate an upper limit of about $2 \mathrm{MeV}$ for the respective spreading width.

\subsection{Neutron and $\gamma$ Decay}

As discussed in chapter 4.2 for ${ }^{136} \mathrm{Xe}$, the neutron decay of the DGDR does not exhibit any particular feature and seems to be governed by statistical decay properties. Detailed studies, however, are still required and presently carried out by the LAND collaboration. 
The DGDR $\gamma$-decay probability, in the case of ${ }^{208} \mathrm{~Pb}$, can be obtained combining the results of (Wajda et al., 1993) and (Ritmann et al., 1993a). In the former study, a DGDR total excitation cross section $\sigma_{\text {total }}$ of $360 \pm 50 \mathrm{mb}$ (preliminary) was measured in the system ${ }^{208} \mathrm{~Pb}+\mathrm{Pb}$ at $650 \mathrm{~A} \cdot \mathrm{MeV}$ bombarding energy, in the latter case a cross section $\sigma_{y y}$ associated to the DGDR double $\gamma$ decay of $0.20(6) \mathrm{mb}$ was obtained for the system ${ }^{208} \mathrm{~Pb}+\mathrm{Bi}$ at $1 \mathrm{~A} \cdot \mathrm{GeV}$ bombarding energy (see Table 3 ). The cross section of the ${ }^{208} \mathrm{~Pb}+\mathrm{Pb}(650 \mathrm{~A}$ $\mathrm{MeV}$ ) measurement can be scaled to the system ${ }^{208} \mathrm{~Pb}+\mathrm{Bi}(1 \mathrm{~A} \cdot \mathrm{GeV})$ without introducing major uncertainities, for example using the folding model (see chapter 3.2), which leads to $490 \pm 70 \mathrm{mb}$. One then obtains the probability $P_{y y}$ for the process of de-excitation via emission of two energetic $\gamma$ rays, presumably with the single GDR as intermediate state,

$$
\mathrm{P}_{y \gamma}=\frac{\sigma_{y \gamma}}{\sigma_{\text {total }}}=(4.1 \pm 1.4) 10^{-4}
$$

Having in mind that the electromagnetic decay probability of a two-phonon state should be that of two independently decaying phonons (see chapter 3 ), one might assume $P_{y y}=\left(P_{y}^{D G D R}\right)^{2}$ where $P_{y}^{D G D R}$ denotes the decay probability of each of the two phonons. From the experimental value of $P_{y y}$ one obtaines $P_{y}^{O G D R}=0.020 \pm 0.004$. This value should be compared to the $\gamma$-decay probability of the single-phonon GDR which experimentally is known to $P_{y}^{G O R}=0.017$ (Beene et al., 1990). Both numbers are well in agreement to each other thus being in favor of the hypothesis of two non-interacting phonons.

\subsection{Cross Sections}

The understanding of cross sections of the double giant dipole resonance, either observed in pion double charge exchange or in heavy ion induced reactions, at present remains as a puzzle. In principle, the semiclassical method for relativistic electromagnetic excitation is well suited to calculate cross sections, if the relevant nuclear structure information is available. Since excitation probabilities can be large in grazing collisions, the problem of coupled channels arises. Up to now, calculations using a coupled channel formalism have not yet been performed. Most of the calculations for DGDR cross sections employ either the folding model or second order perturbation theory. The folding model is restricted to the independent two-phonon excitation in the harmonic limit. This restriction is not present when using second order perturbation theory, but in all practical cases, the assumption of independent phonons was adopted too due to the lack of better knowledge of nuclear structure parameters. Problems related to flux conservation in case of large probabilities are ignored in perturbation theory, while the folding model takes it only into account to the extent that identical phonons are excited. An additional problem arises with the choice of the lower cut-off in impact parameter $b_{\min }$ as discussed in chapter 3 . The choice of $b_{\min }$ in fact is a rather severe source of uncertainities since in heavy ion collisions the excitation probabilities for giant resonances, and even more for two-phonon resonances, peak around $b_{\min }$. Exploring the large data systematics available for one-neutron removal cross sections in heavy ion reactions, in particular on ${ }^{197} \mathrm{Au}$, an attempt was made (Norbury and Baur, 1993) to derive a consistent description for the various systems. Electromagnetic and (small) nuclear contributions were calculated, the former ones involving photo-neutron data, 
Fig. 29. Ratio of experimental to calculated cross sections for the oneneutron removal in ${ }^{197} \mathrm{Au}$ bombarded with different ions (bombarding energies $\leq 2.1$ A.GeV). Results from the analysis of (Norbury and Baur, 1993) and (Aumann et al., 1993) are given by solid and open symbols, respectively. The analysis of (Norbury and Baur, 1993) includes experimental errors and estimates of uncertainities in the calculation, that of (Aumann et al., 1993) only the experimental errors. The data point for the $\mathrm{Pb}\left({ }^{136} \times \mathrm{Xe},{ }^{135} \mathrm{Xe}+1 \mathrm{n}\right)$ is added (see text).

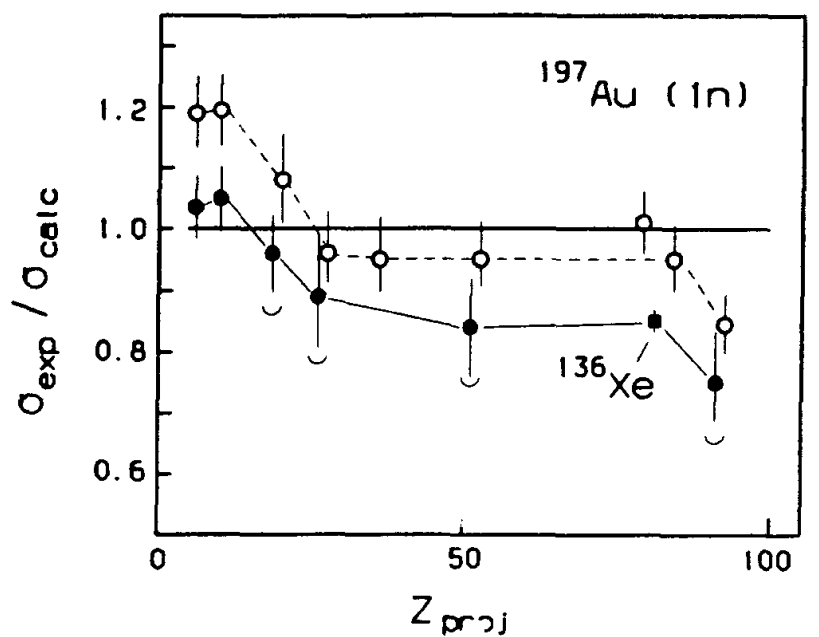

quadrupole excitations, and effects due to the multi-step excitations. A parametrization of $b_{\min }$ (Benesh et al., 1989) was adopted. The result of their analysis is shown in Fig. 29. A tendency of overpredicting the experimental data towards heavier systems is noticed. A parametrization for $b_{\min }$ due to (Kox et al., 1987), which leads to $\sim 10-15 \%$ larger $b_{\min }$ values for heavy systems, was used in an otherwise almost identical analysis due to (Aumann et al., 1993). Their results, also shown in Fig. 29, provide a better agreement for the heavy systems, but underpredict data of lighter systems. A systematic description obviously cannot be achieved, indicating that certain effects are missing in the analysis. A similar analysis for the system $\mathrm{Pb}\left({ }^{136} \mathrm{Xe},{ }^{135} \mathrm{Xe}+1 \mathrm{n}\right.$ ) was performed in (Llope and Braun-Munzinger, 1992) using a choice of $b_{\min }$ according to (Benesh et al., 1989). If combined with the experimental value of (Schmidt, 1991), a value is obtained which fits well into the systematics of (Norbury and Baur, 1993) as shown in Fig. 29.

In the following, experimental cross sections for the double giant dipole resonance are compared with various calculations, summarized in Table 4. A number of calculations was performed for ${ }^{136} \mathrm{Xe}$ aiming on a comparison to the data of (Schmidt et al., 1993). Llope and Braun-Munzinger (1993) used their folding model, while (Ponomarev et al., 1993) and (Bertulani and Zelevinsky, 1993) applied second-order perturbation theory. All calculations are based on the assumption that two phonons are excited independently. The single phonon E1 strength distribution was derived from photo-neutron data, except in (Ponomarev et al., 1993) who used their RPA calculation discussed in the previous section. The various approaches differ also in the choice of $b_{\min }$. Bertulani and Zelevinsky have analyzed the cross sections for the DGDR $1^{n}=0^{+}, 2^{+}$spin multiplett separately and find a ratio of $i: 5.4$ in favor of the $2^{+}$state; in Table 4 the sum of both components is quoted. They also pointed out that the E1 matrix elements are inversely proportional to the phonon energy. Because of the slight anharmonicity found for the DGDR states (see section 5.2 and Fig. 24), this leads to an increase of the DGDR cross 
Table 4. Experimental DGDR cross sections from heavy ion data for ${ }^{136} \mathrm{Xe},{ }^{197} \mathrm{Au}$ and ${ }^{208} \mathrm{~Pb}$ in comparison to various calculations. The values of $b_{\min }$ used in the calculations are also given.

\begin{tabular}{|c|c|c|c|c|}
\hline Nucleus & $\begin{array}{c}\sigma(\mathrm{DGDR}) \\
{[\mathrm{mb}]}\end{array}$ & $\begin{array}{l}b_{\min } /\left(A_{1}^{1 / 3}+A_{2}^{1 / 3}\right) \\
{[f \mathrm{~mm}]}\end{array}$ & Method & Reference \\
\hline${ }^{136} \mathrm{Xe}$ & $\begin{array}{l}215(50) \\
110 \\
182 / 90 \\
130 / 50\end{array}$ & $\begin{array}{l}1.3 \\
1.2 / 1.4 \\
1.2 / 1.5\end{array}$ & $\begin{array}{l}\text { Experimental } \\
\text { Folding Model } \\
\text { Pert. Theory } \\
\text { Pert. Theory }\end{array}$ & $\begin{array}{l}\text { Schmidt et al., } 1993 \\
\text { Llope and Braun-Munzinger, } 1992^{1} \\
\text { Bertulani and Zelevinsky, } 1993 \\
\text { Ponomarev et al., } 1993\end{array}$ \\
\hline${ }^{208} \mathrm{~Pb}$ & $\begin{array}{l}360(50) \\
390 / 200\end{array}$ & $1.2 / 1.4$ & $\begin{array}{l}\text { Experimental } \\
\text { Pert. Theory }\end{array}$ & $\begin{array}{l}\text { Wajda et al., } 1993 \text { (preliminary) } \\
\text { Bertulani, } 1993 \mathrm{~b}\end{array}$ \\
\hline${ }^{197} \mathrm{Au}$ & $\begin{array}{l}430(110) \\
131\end{array}$ & 1.43 & $\begin{array}{l}\text { Experimental } \\
\text { Folding Model }\end{array}$ & $\begin{array}{l}\text { Aumann et al., } 1993 \text { (see text) } \\
\text { Aumann et al., } 1993\end{array}$ \\
\hline
\end{tabular}

1 results were scaled to $700 \mathrm{~A} \cdot \mathrm{MeV}$

sections in the order of about $10 \%$. The single step E2 excitation of the $1^{\pi}=2^{+}$DGDR member was also considered (Bertulani and Zelevinsky, 1993), but was found to be negligible (see also chapter 5.2). Preliminary results for ${ }^{208} \mathrm{~Pb}$ (Wajda et al., 1993) are included in Table 4 as well as data for ${ }^{197} \mathrm{Au}$ obtained from the inclusive measurement of (Aumann et al., 1993). In their analysis they observe an excess yield in the two- and three-neutron removal channel (see chapter 4.1) which we have tentatively assigned as additional DGDR cross section.

From Table 4 it becomes evident that experimental and calculated DGDR cross section can be brought into reasonable agreement, if $b_{\min } \approx 1.2\left(A_{1}^{1 / 3}+A_{2}^{1 / 3}\right)$ is chosen. Such a choice, however, yields too large cross sections for the single-step excitations. The analysis of those, as seen for instance in Fig. 29 , favours a value of $b_{\min } \approx 1.4$ $\left(A_{1}^{1 / 3}+A_{2}^{1 / 3}\right)$ in heavy systems. Such a choice underestimates the DGDR cross sections by a factor $\sim 2-3$. Measurements for ${ }^{208} \mathrm{~Pb}$ performed at low bombarding energies (Beene, 1993; see chapter 4.3) report an excess in cross section by a factor 2 when compared to calculations using the folding model (see Fig. 20). In their analysis it was adopted that the $\gamma$-decay branch of the two-phonon state is identical to that of the GDR. As shown in chapter 5.3, this assumption can be verified. It is pointed out, that in contrast to experiments performed at higher bombarding energies, the excitation probabilities per collision are rather small in this measurement and therefore perturbation theory should be well applicable.

The question whether the discrepancies between calculated and measured cross sections result from an incomplete description of the reaction mechanism, cannot be answered satisfactorily at present. The guidelines for a clarification, however, are obvious: the description of the excitation process can be improved treating single- and multi-step excitations consistently in a coupled channel approach; one might also explore if Coulomb-nuclear interferences play a larger role than expected. On the experimental side, differential cross section measurements with regard to impact parameter are needed. Such experiments are difficult to perform because of the extremely small 
scattering angles involved (see Fig. 6). The LAND collaboration at GSI is presently preparing such experiments utilizing beams of high brilliance after electron cooling.

On the other hand, one can also find arguments that the cross section enhancement observed for the DGDR relative to the GDR has its origin in the nuclear structure of the two-phonon state. More or less, present day calculations rely on the independency of the two phonons. Both, the DGDR resonance energy and width deviate, although slightly, from this picture, indicative of non-linear effects. Moreover, an enhancement in DGDR cross section seems also to be present when the double-phonon state is excited in $\left(\pi^{+}\right.$, $\pi^{-}$) double charge exchange reactions as was already discussed in chapter 4.4. The CCIA calculations have to be renormalized by at least a factor two in order to reproduce the larger experimental cross sections (Fortune, 1991). The missing $\Delta \mathrm{L}=0$ strength is another puzzling question which awaits a solution. In the measurement of the twophonon isoscalar giant quadrupole resonance (Frascaria et al., 1993; see chapter 4.5), the experimental cross section exceeds that of the reaction model of (Blumenfeld and Chomaz, 1988) by approximately a factor 2 , although experimentally not firmly established. In this case the excitation is predominantly due to nuclear interaction.

One might argue that cross section enhancements are observed independent of the excitation mechanism and are thus related to the structure of the two-phonon states. In that case, it would imply that the excitation of the first phonon modifies the mean field or the residual interactions in a way that the second phonon is excited more strongly.

\section{SUMMARY AND OUTLOOK}

Heavy ion inelastic scattering at near relativistic energies is a promising new method in the field of giant resonance studies. Large cross sections giving rise to multi-step excitations and a favorable resonance signal to background ratio are the essential features. Novel experimental techniques were developed in order to cope with the new scenario at relativistic energies.

Until now, the major progress was achieved in exploring the structure of two-phonon states of the isovector giant dipole resonance. The results from heavy ion scattering are consistent with results from pion double charge exchange reactions. The two-phonon isoscalar giant quadrupole resonance was also found in a measurement with heavy ions at lower bombarding energy. The observation of multiple giant resonance excitations was a missing piece in the spectrum of collective nuclear excitations, the non-existence of which would have cast doubt upon our understanding of nuclear giant resonances.

As predicted theoretically for the highly collective small amplitude giant resonance motion, the energy of the observed two-phonon states exhibit only very small deviations from the harmonic limit, i.e.

$$
E(D G D R) \approx 2 E(G D R) .
$$

This observation is indicative of two phonons, which interact only weakly, a fact which is also supported by the apparent width of the two-phonon states. 
The width is observed experimentally at values between the two limits proposed theoretically

$$
\sqrt{2} \Gamma(G D R)<\Gamma(D G D R)<2 \Gamma(G D R),
$$

indicating a small spreading into non-resonant compound states.

Details remain to be understood, in particular the observed strength appears to be larger than expected. Since the electromagnetic reaction mechanism, in principle, can be described accurately, the discrepancies found for the heavy ion induced two-phonon giant dipole cross sections demand a solution. The methods of appropriate data analysis, i.e. extraction of transition strength, as well as the experimental methods can still be improved, giving hope that achieve a complete understanding will be achieved.

A cross section enhancement of the same order, however, is observed in pion reactions as well and is also indicated in nuclear excitation of the two-phonon isoscalar giant quadrupole resonance. One might thus argue, that the excess in cross section results from features related to the structure of the multi-phonon resonances, which are not covered in present day theories.

As the two-phonon GDR states show up as a clear signal in relativistic heavy scattering, one can hope that even higher-phonon states of the giant dipole resonance will be found experimentally. In one of the experiments, hints at a three-phonon state might be recognized, but this still needs an experimental confirmation. Higher-phonon states are the signposts in an exploration of the nuclear response to collective large amplitude motions, and their observation would allow for a detailed study of non-linear effects.

Experiments of the type described in this article are feasible even using secondary radioactive ion beams, now available at a number of heavy ion accelerator facilities. Studies of giant resonances, in particular of electric dipole and quadrupole excitations, can thus be carried out for nuclei of extreme isospin, and eventually new phenomena, non-existent in stable isotopes, could be o'sserved.

I am very much indebted to Mrs. A. Busch for preparing the manuscript, to $S$. Mojumder for a careful reading of this report, and to my colleagues $C$. A. Bertulani and J. Stroth at GSI for many valuable discussions.

I appreciate the support from my colleagues of the LAND collaboration from the Universities Bochum, Cracow, Frankfurt, Mainz, and from GSI.

My special thanks for providing their latest results and for clarifying discussions are due to J.R. Beene from Oak Ridge, N. Frascaria from Orsay, S. Mordechai from Beer-Sheva, T. Aumann and K. Sümmerer from GSI, W. Kühn and J.L. Ritman from Gießen. 


\section{REFERENCES}

Akyüz R.Ö and S. Fallieros, Phys. Rev. Lett. 22, 1016 (1971)

Alarcon R., P.L. Cole, D.S. Dale, P.T. Debevec, and L.J. Morford, Phys. Rev. C43, R2470 (1991)

Alder K. and A. Winther, Coulomb Excitation, Academic Press, New York and London (1966)

Alder K. and A. Winther, Electromagnetic Excitation, North-Holland, Amsterdam (1975)

Anderson J.D., C. Wong, and J.W. McClure, Phys. Rev. 138B, 615 (1965)

Antony M.S., J. Britz, J.B. Buch, and A. Pape, At. Data Nucl. Data Tables 33, 4471 (1985)

Aumann T., J.V. Kratz, E. Stiel, K. Sümmerer, W. Brüchle, M. Schädel, G. Wirth, M. Fauerbach, and J.C. Hill, Phys.Rev. C47, 1728 (1993)

Aumann T., J.V. Kratz, E. Stiel, K. Sümmerer, W. Brüchle, M. Schädel, G. Wirth, M. Fauerbach, and J.C. Hill, Nucl. Phys. Conf. on Giant Resonances, Gull Lake (1993), to be publ. in Nucl. Phys. A

Baldwin G.C. and G.S. Klaiber, Phys. Rev. 71, 3 (1947); Phys. Rev. 73, 1156 (1948)

Barrette J., N. Alamanos, F. Auger, B. Fernandez, A. Gillibert, D.J. Horen, J.R. Beene, F.E. Bertrand, R.L. Auble, B.L. Burks, J.G. Gomez del Campo, M.L. Halbert, R.O. Sayer, W. Mittig, Y. Schutz, B. Haas, and J.P. Vivien, Phys. Lett. 209B, 182 (1988)

Barrette J., P. Braun-Munzinger, W.E. Cleland, G. David, E. Duek, M. Fatyga, D. Fox, S.V. Greene, J.R. Hall, R. Heifetz, T.K. Hemmick, N. Herrmann, R.W. Hogue, G. Ingold, K. Jayananda, D. Kraus, A. Legault, D. Lissauer, W.J. Llope, T. Ludlam, R. Majka, D. Makowiecki, S.K. Mark, J.T. Mitchell, M. Muthuswamy, E. O'Brien, L.H. Olsen, V. Polychronakos, M. Rawool-Sullivan, R.S. Rotondo, J. Sandweiss, B. Shivakumar, J. Simon, U. Sonnadara, J. Stachel, J. Sunier, H. Takai, T.G. Throwe, H. Van Hecke, L. Waters, W.J. Willis, K. Wolf, D. Wolfe, and C.L. Woody, Phys. Rev. C41, 1512 (1990)

Bar-Tour J., and S. Mordechai, Phys. Rev. C45, 197 (1992)

Beene J.R., F.E. Bertrand, D.J. Horn, R.L. Auble, B.L. Burks, J. Gomez del Campo, M.L. Halbert, R.O. Sayer, W. Mittig, Y. Schutz, J. Barrette, N. Alamanos, F. Auger, B. Fernandez, A. Gillibert, B. Haas, and J.P. Vivien, Phys. Rev. C41, 920 (1990)

Beene J.R., Int. Nucl. Phys. Conf. on Giant Fesonances, Gull Lake (1993), to be published in Nucl. Phys. A

Benesh C.J., B.C. Cook, and J.P. Vary, Phys. Rev. C40, 1198 (1989)

Bermann B.L., At. Data Nucl. Data Tables 15, 319 (1975)

Berman B.L. and S.C. Fultz, Rev. Mod. Phys. 47. 7131 (1975)

Bertrand F.E., Annu. Rev. Nucl. Sci. 26, 457 (1976)

Bertrand F.E., R.O. Sayer, R.L. Auble, M. Beckermann, J.L. Blankenship, B.L. Burks, M.A.G. Fernandez, C.W. Glover, E.E. Gross, D.J. Horen, J. Gomez del Campo, and D. Shapira, Phys. Rev. C35, 111 (1987)

Bertulani C.A. and G. Baur, Phys. Rep. 163, 299 (1988)

Bertulani C.A. and V. Zelevinsky, Phys. Rev. Lett. 71, 967 (1993); Nucl. Phys. A, in press

Bertulani C.A., priv. comm. (1993)

Blaich Th., Th.W. Elze, H. Emling, H. Freiesleben, K. Grimm, W. Henning, R. Holzmann, G. Ickert, J.G. Keller, H. Klingler, W. Kneissl, R. Konig, R. Kulessa, J.V. Kratz, D. Lambrecht, J.S. Lange, Y. Leifels, E. Lubkiewicz, M. Proft, W. Prokopowicz, C. Schütter, R. Schmidt, H. Spies, K. Stelzer, J. Stroth, W. Walus, E. Wajda, H.J. Wollersheim, M. Zinser, and E. Zude, (LAND Collaboration), Nucl. Instr. and Meth. in Physics Research A314, 136 (1992)

Blomquist J., Phys. Lett. B33, 541 (1970)

Blumenfeld Y. and Ph. Chomaz, Phys. Rev. C38, 2157 (1988)

Bohr A. and B.R. Mottelson, Nuclear Structure Vol. II, W.A. Benjamin, Inc. (1975)

Bothe W. and W. Gentner, Z. Phys. 106, 236 (1937) 
Brink D., Thesis, Oxford University (1955)

Buenerd M., J. Chauvin, G. Duhamel, J.Y. Hostachy, D. Lebrun, P. Martin, P.O. Pellegrin, G. Perrin, and P. de Saintignon, Phys. Lett. 167B, 379 (1986)

Catara F., Ph. Chomaz, and N. Van Giai, Phys. Lett. B233, 6 (1989)

Cardman L.S., Nucl. Phys. A345, 173c (1981)

Chomaz Ph., N. Frascaria, Y. Blumenfeld, J.P. Garron, J.C. Jacmart, J.C. Poynette, W. Bohne, A. Gamp, W. von Oertzen, M. Buenerd, D. Lebrun, and Ph. Martin, Z. Phys. A318, 41 (1984)

Chomaz Ph., Y. Blumenfeld, N. Frascaria, J.P. Garron, J.C. Jacmart, J.C. Roynette, W. Bohne, A. Gamp, W. von Oertzen, Nguyen Van Giai, and D. Vautherin, Z. Phys. A319, 167 (1984)

Chomaz Ph. and N. Van Giai, Phys. Lett. B282, 13 (1992)

Clement H., Prog. Part. Nucl. Phys., 29, 175 (1992)

Czosnyka T., D. Cline, and. C.Y. Wu, Bull. Am. Phys. Soc. 28, 745 (1983)

Dowell D.H., G. Feldman, K.A. Snover, A.M. Sandorfi, and M.T. Collins, Phys. Rev. Lett. 50, 1191 (1983)

Eisenberg J.M. and W. Greiner, Nuclear Models, North-Holland, Amsterdam (1975)

Emling $H$., Workshop on experiments at external beam lines of SIS and ESR, GSI Report 85-24, Darmstadt, p. 85 (1985)

Emling H., Nucl. Phys. A520, 687c (1990)

Emling H., Nucl. Phys. A533, 493c (1993)

Emling H., Physikalische Blatter $\underline{3,} 201$ (1993)

Fallieros S., and B. Goulard, Nucl. Phys. A147, 593 (1970)

Fermi E., Z. Phys. 29, 315 (1924)

Fortune H.T., Nucl. Phys. A522, 241c (1991)

Frascaria N., Nucl. Phys. A482, 245a (1988)

Frascaria N., Int. Nucl. Phys. Conf. on Giant Resonances, Gull Lake (1993), to be published in Nucl. Phys. A

Gaardhoje J.J., Ann. Rev. Nucl. Part. Science 42, 483 (1992)

Grein H., H. Emling, and J. Stachel, Scientific Report, GSI Darmstadt (1983)

Goldhaber M. and E. Teller,Phys. Rev. 74, 1046 (1948)

Gomes A.C.V. and C.A. Bertulani, Nucl. Phys. 517, 639 (1990)

Grof Y., N. Auerbach, D. Benton, H.T. Fortune, K. Johnson, M.A. Kagarlis, G. Kahrimanis, S. Mordechai, C.L. Morris, J.M. O'Donnell, I. Orion, D. Saunders, D.A. Smith, H. Ward, and C. Fred Moore, Phys. Rev. C47, 1466 (1993)

Hill J.C., F.K. Wohn, J.A. Winger, M. Khayet, and K. Leininger, Phys. Rev. C38, 1722 (1988)

Jackson J.D., Classical Electrodynamics, Wiley, New York (1975)

Jäckle R. and H. Pilkuhn, Nucl. Phys. A247, 521 (1975)

Jang S., Nucl. Phys. A401, 303 (1983)

Kamermans R., J. van Driel, H.P. Morsch, J. Wilczynski, and A. van der Woude, Phys. Lett. B82, 221 (1979)

Kaufmann M.W. Weisfield, E.P. Steinberg, B.D. Wilkins, and D. Henderson, Phys. Rev. C14, 1121 (1976)

Kaufmann S.B. and E.P. Steinberg. Phys. Rev. C22, 167 (1980)

Kaufmann S.B., E.P. Steinberg, B.D. Wilkins, and D.J. Henderson, Phys. Rev. C22, 1897 (1980)

Korten W., T. Härtlein, J. Gerl, D. Habs, and D. Schwalm, Phys. Lett. B317, 19 (1993)

Kox S., A. Gamp. C. Perrin, J. Arvieux, R. Bertholet, J.F. Bruandet, M. Buernerd, R. Cherkaoui, A.J. Cole, Y. El-Masri, N. Longequeue, J. Menet, F. Merches, and J.B. Vivien, Phys. Rev. C35, 1678 (1987)

Kühn W., Int. Nucl. Phys. Conf. on Giant Resonances, Gull Lake, USA (1993), to be published in Nucl. Phys. A

LAND Collaboration, GSI Nachrichten 09-91, GSI Darmstadt (1991) 
Lauritsch G. and P.-G. Reinhard, Nucl. Phys. A509, 287 (1990)

Levinger J.S.,Nuclear Photo-Disintegration, Oxford University Press, Oxford (1960)

Lewis M.B. and F.E. Bertrand, Nucl. Phys. A196, 337 (1972)

Llope W. and P. Braun-Munzinger, Phys. Rev. C41, 644 (1990)

Llope W. and P. Braun-Munzinger, Phys. Rev. C45, 799 (1992)

Matzdorf R., G. Mehler, and G. Soff, Z. Phys. D6, 5 (1987)

Matzdorf R., B. Fricke, and G. Soff, Z. Phys. D17, 233 (1990)

Mercier M.T., J.C. Hill, F.K. Wohn, C.M. McCullough, M.E. Nieland, J.A. Winger, C.B. Howard, S. Renwick, D.K. Matheis, and A.R. Smith, Phys. Rev. C33, 1655 (1986)

Merzbacher E., Quantum Mechanics, 2nd Ed., Wiley, New York (1970)

Mordechai S., N. Auerbach, M. Burlein, H.I. Torune, S.J. Greene, C. Fred Moore, C.L. Morris, J.M. O'Donell, M.W. Rawool, J.D. Silk, D.L. Watson, S.H. Yoo, and J.D. Zumbroo, Phys. Rev. Lett. 61,531 (1988)

Mordechai S., H.T. Fortune, J.M. O'Donell, G. Liu, M. Burlein, A.H. Waosmaa, S. Greene, C.L. Morris, N. Auerbach, S.H. Yoo, and C. Fred Moore, Phys. Rev. C41, 202 (1990)

Mordechai, S. and C. Fred Moore, Nature 323, 393 (1991)

Mordechai S. and C. Fred Moore, Int. Nucl. Phys. Conf. on Giant Resonances, Gull Lake (1993), to be published in Nucl. Phys. A

Myers W.D. and W.J. Swiatecki, Ann. Phys. N.Y. 84, 186 (1974)

Myers W.D., W.J. Swiatecki, T.Kodama, L.J. El-Jaick, and E.R. Hilf, Phys.Rev. C15, 2032 (1977)

Newton J.O., B. Herskind, R.M. Diamond, E.L. Dines, J.E. Draper, K.-H. Lindenberger, C. Schuch, S. Shih, and F.S. Stephens, Phys. Rev. Lett. 46, 1383 (1981)

Novotny R., IEEE Trans. on Nucl. Sci. 38, 379 (1991)

Norbury J.W. and G. Baur, Phys. Rev. C48, 1915 (1993)

Ower H., Th. W. Elze, J. Idzko, K. Stelzer, H. Emling, P. Fuchs, D. Schwalm, H. J. Wollersheim, N. Kaffrell, and N. Trautmann A388 421 (1982)

Pitthan R. and Th. Walcher, Phys. Lett. 36B, $563(1971)$

Pitthan R., Nukleonika 24, 399 (1979)

Ponomarev V. Yu and V.V. Voronov, Phys. Lett. B279, 1 (1992)

Ponomarev V. Yu and V.V. Voronov, Int. Conf. on Nucl. Structure at low and intermediate energies, Dubna (1992)

Ponomarev V. YU, E. Vigezzi, P.F. Bortignon, R.A. Broglia, G. Coläo, G. Lazzari, V.V. Voronov, and G. Baur, preprint (1993), subm. to Phys. Rev. Lett.

Reinhard P.-G. and J. Friedrich, Z. Phys. A321, 619 (1985)

Ritman J.L., F.-O. Berg, W. Kühn, V. Metag, R. Novotny, M. Notheisen, P. Paul, M. Pfeiffer, O. Schwalb, H. Löhner, L. Venema, A. Gobbi, N. Herrmann, K.D. Hildenbrand, J. Mösner, R.S. Simon, K. Teh, J.P. Wessels, and T. Wienold, Phys. Rev. Lett. 70, 533 (1993) and erratum, Phys. Rev. Lett. 70, 2659 (1993)

Ritman J.L., thesis, University Stony Brook, GSI Report 93-20 (ISSN 0171-4546), Darmstadt (1993)

Satchler G.R., Direct Nuclear Interactions, Clarendon Press, Oxford (1983)

Scarpaci J.A., Y. Blumenfeld, Ph. Chomaz, N. Frascaria, J.P. Garron, J.C. Roynette, T. Suomijarrvi, N. Alamanos, B. Fernandez, A. Gillibert, A. Lepine, A. van der Woude, Phys. Rev. Lett. 71. 3766 (1993)

Schelhaas K.P., J.M. Henneberg, M. Sanzone-Arenhövel, N. Wieloch-Laufenberg, U. Zurmühl, B. Ziegler, M. Schumacher, and F. Wolf, Nucl. Phys. A489, 189 (1988)

Schiff L.I., Quantum Mechanics, McGraw-Hill, New York (1968)

Schmidt R., thesis, Universität Mainz, GSI Report 91-35 (ISSN 0171-4546), Darmstadt (1991)

Schmidt R., Th. Blaich, Th. W. Elze, H. Emling, H. Freiesleben, K. Grimm, W. Henning, R. Holzmann, J.G. Keller, H. Klingler, R. Kulessa, J.V. Kratz, D. Lambrecht, J.S. Lange, Y. Leifels, E. Lubkiewicz, E.F. Moore, E. Wajda, W. Prokopowicz, Ch. Schütter, H. Spies, K. 
Stelzer, J. Stroth, W. Walus, H.J. Wollersheim, M. Zinser, and E. Zude, (LAND Collaboration), Phys. Rev. Lett. 70, 1767 (1993)

Scholtz F.G. and F.J.W. Hahne, Z. Phys. A336, 145 (1990)

Smith D.A., H.T. Fortune, J.M. O'Donell, J.D. Silk, B. Gluckmann, R. Crittenden, N. Claytor, C.L. Morris, and J.A. McGill, Phys. Rev. C47, 1053 (1993)

Snover K.A., Ann. Rev. Nucl. Part. Sci. 36, 1 (1986)

Soloviev V.G., Ch. Stoyanov, and A.I. Vdovin, Nucl. Phys. A288, 376 (1977)

Sonnadara U., Int. Nucl. Phys. Conf. on Giant Resonances, Gull Lake (1993), to be publ. in Nucl. Phys. A

Speth J., ed., International Review of Nuclear Physics, Vol. Z, World Scientific (1991)

Spies H., thesis, Universität Frankfurt, GSI Report 92-12 (ISSN 0171-4546), Darmstadt (1992)

Steinwedel $H$. and J.H.D. Jensen, Z. Naturforsch. 5a, 413 (1950)

Suomijärvi T., D. Beaumel, Y. Blumenfeld, Ph. Chomaz, N. Frascaria, J.P. Garron, J.C. Roynette, J.A. Scarpaci, J. Barrette, B. Fernandez, J. Gastebois, and W. Mittig, Nucl. Phys. A509, 369 (1990)

Stroth J. and E. Wajda, priv. comm., GSI (1993)

Tassie L.J., Austr. J. Phys. A305, 509 , 1978)

Typel S. and G. Baur, preprint KfA-IKP (TH)-22 (1993)

van der Woude A., Prog. Part. Nucl. Phys. 18, 217 (1987)

van der Woude A., Int. Review of Nucl. Phys. Z, 100 (1991)

Veyssière S., H. Beil, R. Bergère, P. Carlos, and A. Lepêtre, Nucl. Phys. 159, 561 (1970)

Vretenar D., H. Berghammer, and P. Ring, Phys. Lett. B319, 29 (1993)

Wajda E., J. Stroth, Th. Blaich, Th.W. Elze, H. Emling, H. Freiesleben, K. Grimm, W. Henning, R. Holzmann, H. Klingler, R. Kulessa, J.V. Kratz, D. Lambrecht, Y. Leifels, E. Lubkiewicz, E.F. Moore, K. Stelzer, W. Walus, M. Zinser, and E. Zude, Int. Nucl. Phys. Conf. on Giant Resonances, Gull Lake (1993), to be publ. in Nucl. Phys. A

Weizsäcker C.F., Z. Phys. $88,612(1934)$

Williams E.J., Phys. Rev. 45, 729 (1934)

Williams E.J., K. Dan. Vidensk, Selsk. Mat.-Fys. Medd. 13, 4 (1935)

Winther A. and J. de Boer, in Coulomb Excitation, Academic Press, New York and London (1966) Winther A. and K. Alder, Nucl. Phys. A319, 518 (1979)

Wollersheim H.J., P. Egelhof, H. Emling, J. Gerl, W. Henning, R. Holzmann, R. Schmidt, R.S. Simon, V. Martin, G. Eckert, Th.W. Elze, K. Stelzer, R. Kulessa, G. Duchene, B. Haas, J.C. Merdinger, J. Vivien, J. de Boer, E. Hauber, K. Kaiser, P. von Brentano, R. Reinhardt, R. Wirowski, R. Julin, C. Fahlander, I. Thorslund, and H. Kluge, Z. Phys. A341, 137 (1992) 